FERMILAB-TM-2242

\title{
MARS Simulations of the NuMI Primary Beamline
}

Sergei Striganov

11 May 2004 


\section{Contents}

$\begin{array}{llc}1 & \text { Introduction } & 3\end{array}$

2 MARS model setup $\quad 4$

3 Calculation Procedure $\quad 6$

4 Model Results $\quad 9$

4.1 Effects of Beam Phase Space . . . . . . . . . . . . . . . . . . . . . . . . . . .

4.2 Beam Loss vs. Magnet Current Variations . . . . . . . . . . . . . . . . 14

4.3 Star Density vs. Beam Loss Models . . . . . . . . . . . . . . . . . . . 18

4.4 Multi-wire Beam Loss . . . . . . . . . . . . . . . . . . . . . . . . . . . . . . . . . . . . . . .

4.5 Beam Loss Correlations . . . . . . . . . . . . . . . . 34

$\begin{array}{ll}\text { A MARS Model Elements } & 37\end{array}$

$\begin{array}{lll}\text { B Star Density Distributions } & 48\end{array}$

$\begin{array}{lll}\text { C Star Density and Beam Loss Monitors } & 89\end{array}$ 


\section{Chapter 1}

\section{Introduction}

MARS is a Monte Carlo code for simulation of three-dimensional hadronic and electromagnetic cascades, muon and low-energy neutron transport in shielding and in accelerator and detector components in the energy range from a fraction of an $\mathrm{eV}$ up to $100 \mathrm{TeV}$. This report uses MARS to both transport the $120 \mathrm{GeV}$ primary proton beam from the NuMI extraction Lambertsons through the NuMI Pre-target Hall and calculate the radiological effect of beam losses at various locations and for a variety of conditions. These results are used to:

- anticipate where beam losses will be significant

- determine the level of activation of components

- calculate ground water activation and confirm adequacy of shielding

The results are presented in tables and figures along with drawings of the magnets as they were modeled in MARS. Details of the model elements are found in Appendix A. Further details of beam loss case studies are included in Appendix B. 


\section{Chapter 2}

\section{MARS model setup}

The MARS NuMI beam line model includes the three NuMI Lambertson magnets, one MI lattice quadrupole, four 3Q60 and seventeen 3Q120 quadrupoles, one CMG-1 ("C"-magnet), six EPB, ten B2 bend magnets, two trim dipoles and ten horizontal and nine vertical correctors. A schematic of the major NuMI beamline elements is shown in Figure 2.1. Transverse and longitudinal cross sections of magnets are shown in Appendix A.

Longitudinal cross sections of NuMI tunnel are shown in the Appendix A. Note that the tunnel shape changes many times. Transverse cross sections of tunnel at different places are also presented in Appendix A. 

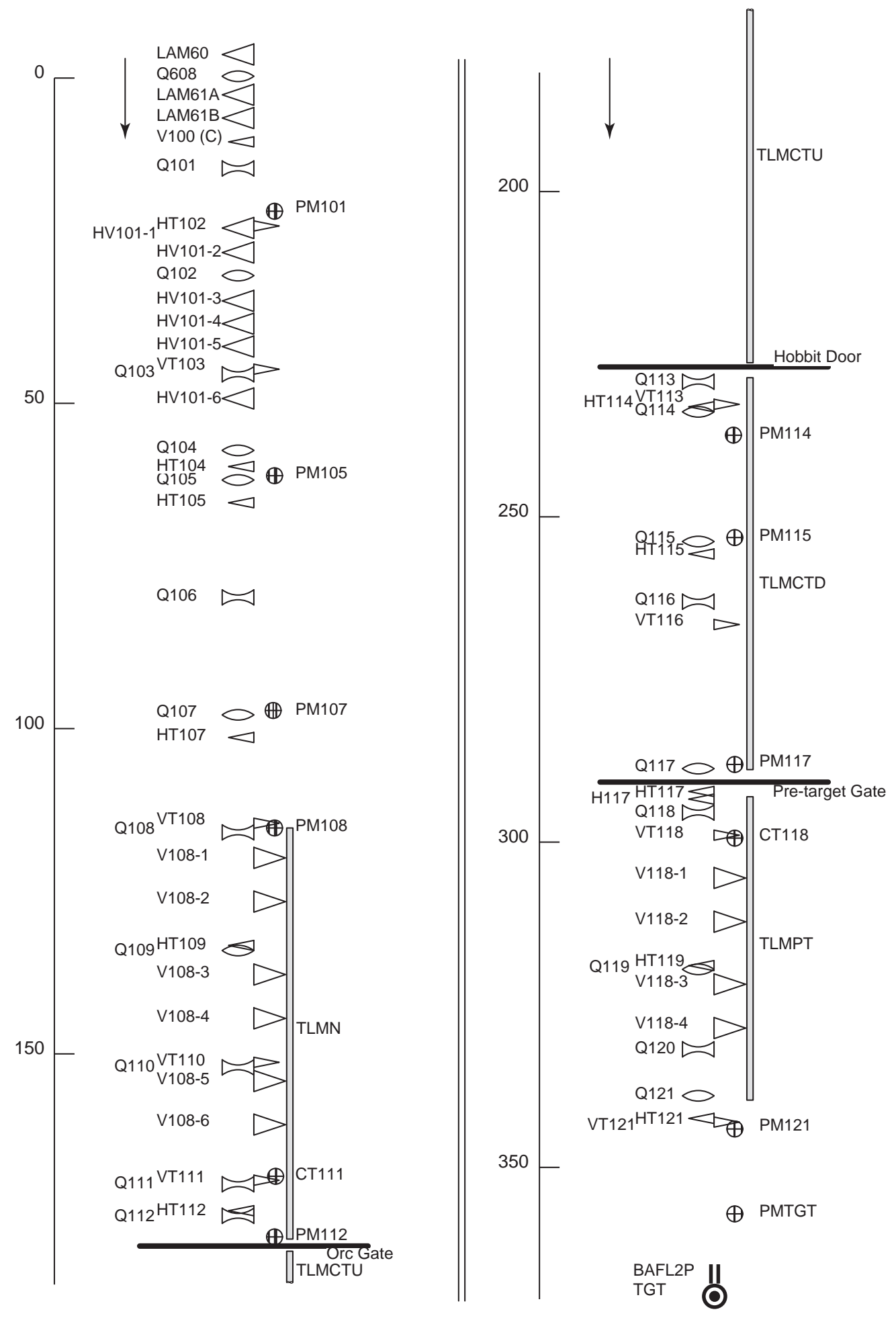

Figure 2.1: Beamline elements in NuMI. The scale is meters. The components designations are: $\mathrm{Q}=$ quadrupole (convexihoriz focus, concave ¿vert focus), $\mathrm{V}=$ vert. dipole, $\mathrm{H}=$ horiz. dipole, $\mathrm{HV}=$ rotated dipole, $\mathrm{VT}=$ vert. trim dipole, $\mathrm{HT}=$ horiz. trim dipole, $\mathrm{LAM}=$ labertson extraction magnet, $\mathrm{PM}=$ position monitor(wire scanner), $\mathrm{CT}=$ calibration target, $\mathrm{TLM}=$ loss monitor. Not shown: 45 sealed loss monitors on each magnet. 


\section{Chapter 3}

\section{Calculation Procedure}

The MARS model of NuMI beamline was used to calculate

1. beam loss, star density

2. energy deposition in beam loss monitors

3. residual activity in beamline components

These calculations were performed for an appropriate set of variations in magnet current and the presence of wire scanners in the beam pipe.

First beam loss was simulated and the distribution of points where beam protons escaped the channel aperture was accumulated. If a beam proton exited the aperture it was stopped without production of secondary particles and was considered "lost". For all scenarios the number of "lost protons" was found for all the magnets and drift spaces. As a check for systematic errors, the simulation of beam loss distributions was performed by using two programs: MARS and STRUCT. Results of the two sets of calculations agree rather well (for example see figure 4.3), but STRUCT executes much faster. Hence, most results on beam loss were obtained using the STRUCT code. However, STRUCT does not provide the option to simulate cascades outside beam pipe. Therefore, star densities, energy deposition and residual activity were calculated using the MARS14 code.

A "star" is simply defined as an hadronic interaction vertex with at least one secondary particle which has a kinetic energy greater than $50 \mathrm{MeV}$. Average star densities were calculated for 7 regions around the NuMI tunnel as shown in figure 3.1. In most cases regions were defined for each section of the tunnel with constant cross section. For each region and any scenario, the maximum of star density distribution in the 
rock near the tunnel (1 cm wide bins were used) are defined. Then the $99.9 \%$ volume boundary was determined by finding the transverse distance where the star density falls to $0.1 \%$ of maximum. For this volume the average star density, average radius and maximum radius are calculated.

In the simulation runs for residual activity a $20 \mathrm{MeV}$ global threshold was used. 


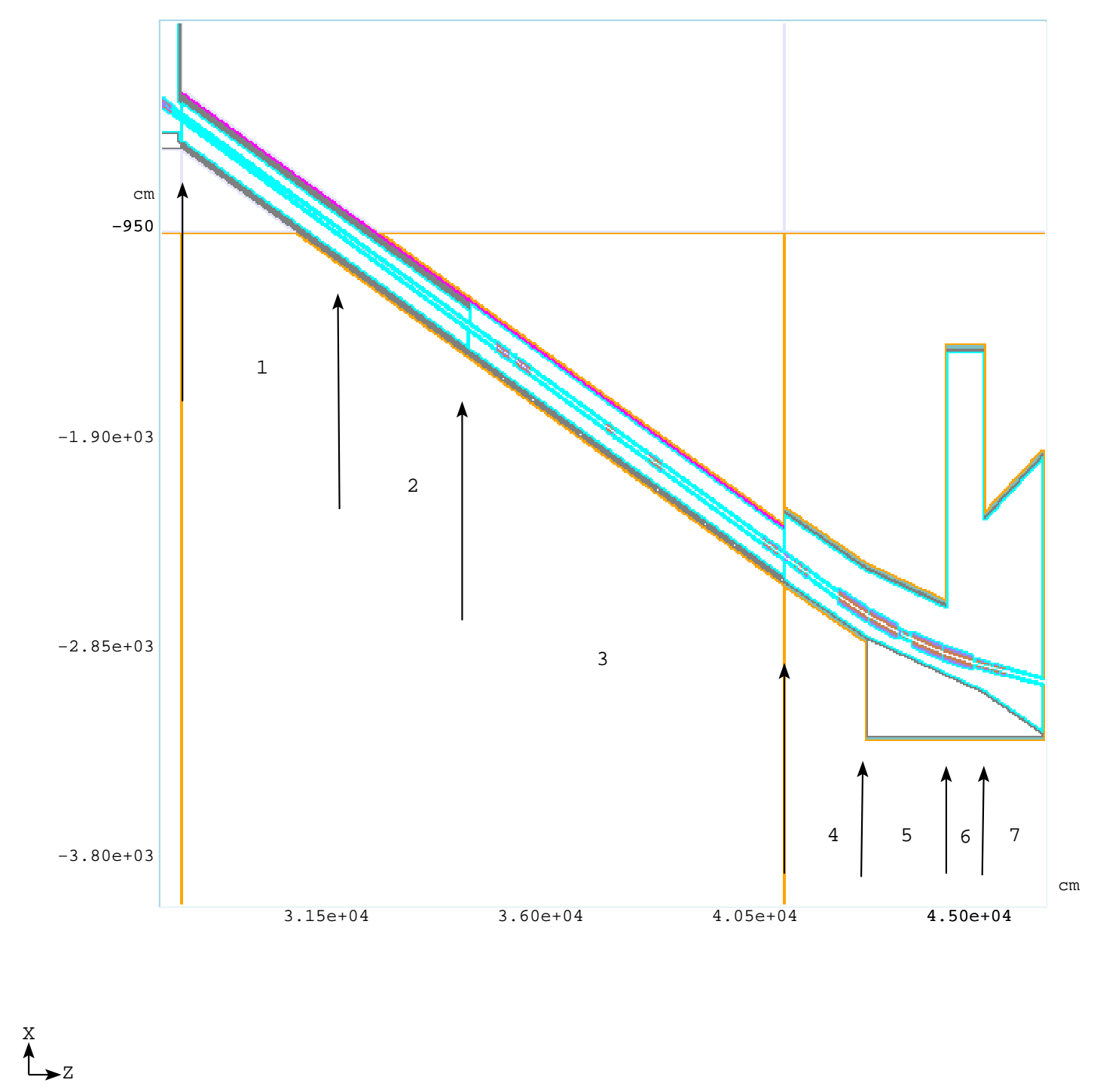

Figure 3.1: Regions for scoring star density distribution. 


\section{Chapter 4}

\section{Model Results}

\subsection{Effects of Beam Phase Space}

The nominal phase space considered is $25 \pi \mathrm{mm} \operatorname{mrad} 95 \%$ emittance with a $500 \pi$ cut on beam tails. Momentum spread is $\delta p / p=4 \times 10^{-4}$ at $95 \%$ with tails extending to $3 \times 10^{-3}$. Phase space distribution at the entrance to the first NuMI extraction Lambertson is shown in figure 4.1. These parameters are based on the measured performance of the Main Injector.

Beam size for $500 \pi$ cut in comparison with beam-line apertures is presented in figure 4.2. It is clearly seen that beam losses for the normal tune are certainly less than $10^{-6}$ per proton (the last column in Table 4.1 ).

Beam profiles calculated using STRUCT and MARS at the end of NuMI channel are compared in figure 4.3. There is small shift of beam center (about $0.1 \mathrm{~mm}$ ) in MARS calculations.

Beam of different emittances, from $25 \pi$ to $60 \pi$, was transported through NuMI primary magnet system using old (prior to 2001) and current design. Resulting beam loss are presented in table 4.1. It is seen that the beam loss level is much lower for the present design. Beam losses only occur on the baffles, which are within the Target Hall shield pile, and are designed to withstand the mis-steering of the beam.

In addition, a worst case condition was simulated in STRUCT using an emittance of $40 \pi \mathrm{mm} \operatorname{mrad}(95 \%)$ with a cut-off at $500 \pi$ and momentum spread $\delta p / p$ of $4 \times 10^{-4}$ with a cut-off at $3 \times 10^{-3}$. The losses at the end of the channel in front of the target (BAFL2P) with these conditions was found to be only $1.3 \times 10^{-6}$ per proton. 
Table.4.1 : Beam loss for normal tune (per primary proton). BAFFLE1 was only a part of the old beamline design)

\begin{tabular}{llllll}
\hline $95 \%$ emittance, $\mathrm{mm} \cdot \mathrm{mrad}$ & $60 \pi$ & $40 \pi$ & $60 \pi$ & $40 \pi$ & $25 \pi$ \\
\hline cuts, $\mathrm{mm} \cdot \mathrm{mrad}$ & no & no & no & no & $500 \pi$ \\
\hline$\delta p / p$ & $4 \times 10^{-4}$ & $2 \times 10^{-4}$ & $4 \times 10^{-4}$ & $2 \times 10^{-4}$ & $4 \times 10^{-4}$ \\
\hline design & old & old & new & new & new \\
\hline HT107 & $2.0 \times 10^{-3}$ & $6.0 \times 10^{-5}$ & 0. & 0. & 0. \\
V110-1 & $8.7 \times 10^{-4}$ & 0. & 0. & 0. & 0. \\
HT108 & $1.7 \times 10^{-3}$ & $2.0 \times 10^{-3}$ & 0. & 0. & 0. \\
BAFFLE1 & $4.0 \times 10^{-3}$ & $1.8 \times 10^{-3}$ & NA & NA & NA \\
BAFL2P & $3.4 \times 10^{-3}$ & $5.2 \times 10^{-4}$ & $1 . \times 10^{-4}$ & $9 . \times 10^{-7}$ & 0. \\
\hline total & $1.2 \times 10^{-2}$ & $4.4 \times 10^{-3}$ & $1 . \times 10^{-4}$ & $9 . \times 10^{-7}$ & 0. \\
\hline
\end{tabular}



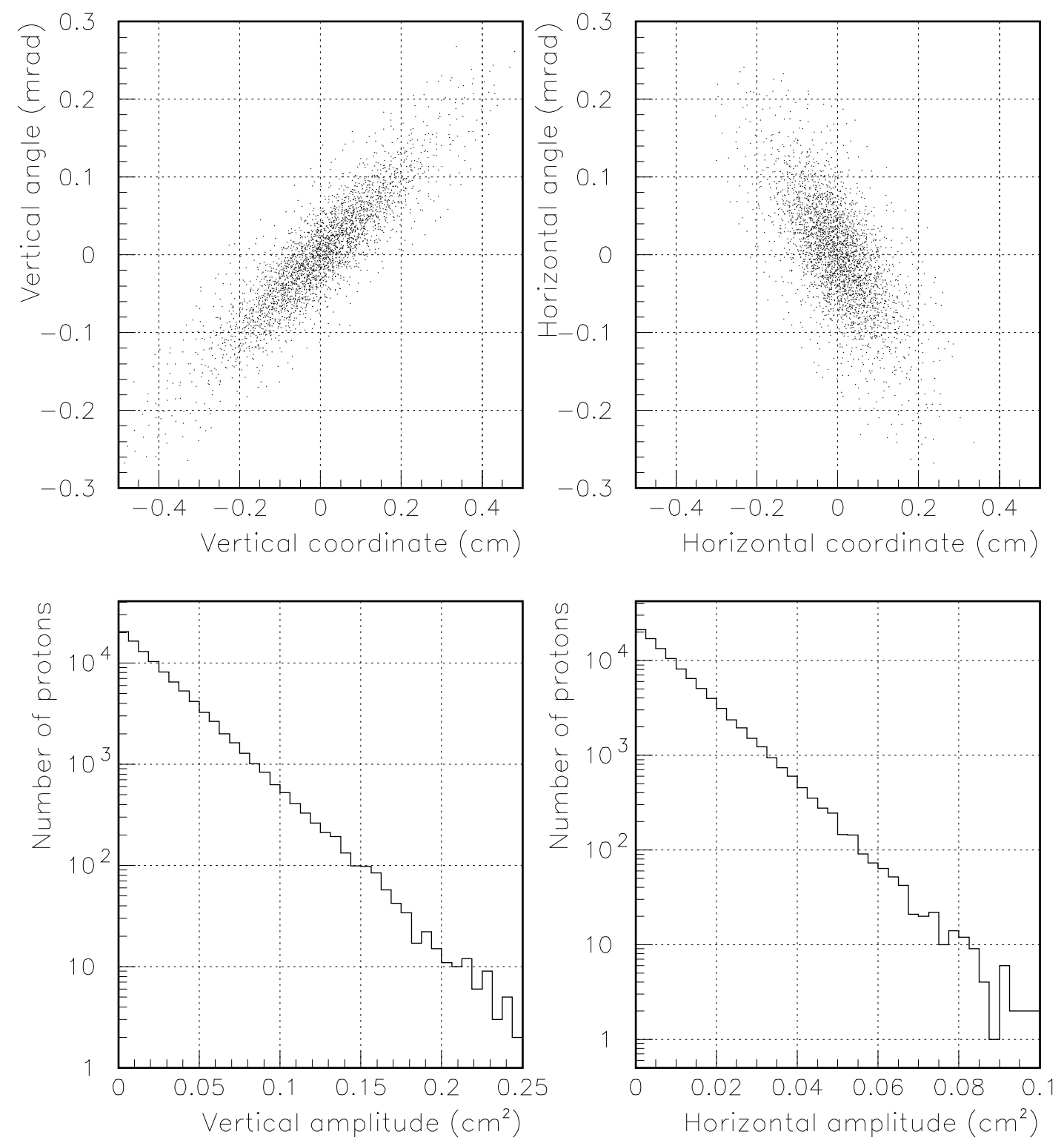

$25 \pi \mathrm{mm}$ mrad $95 \%$ emittence with $500 \pi$ cut

Figure 4.1: Beam distribution at first Lambertson. 


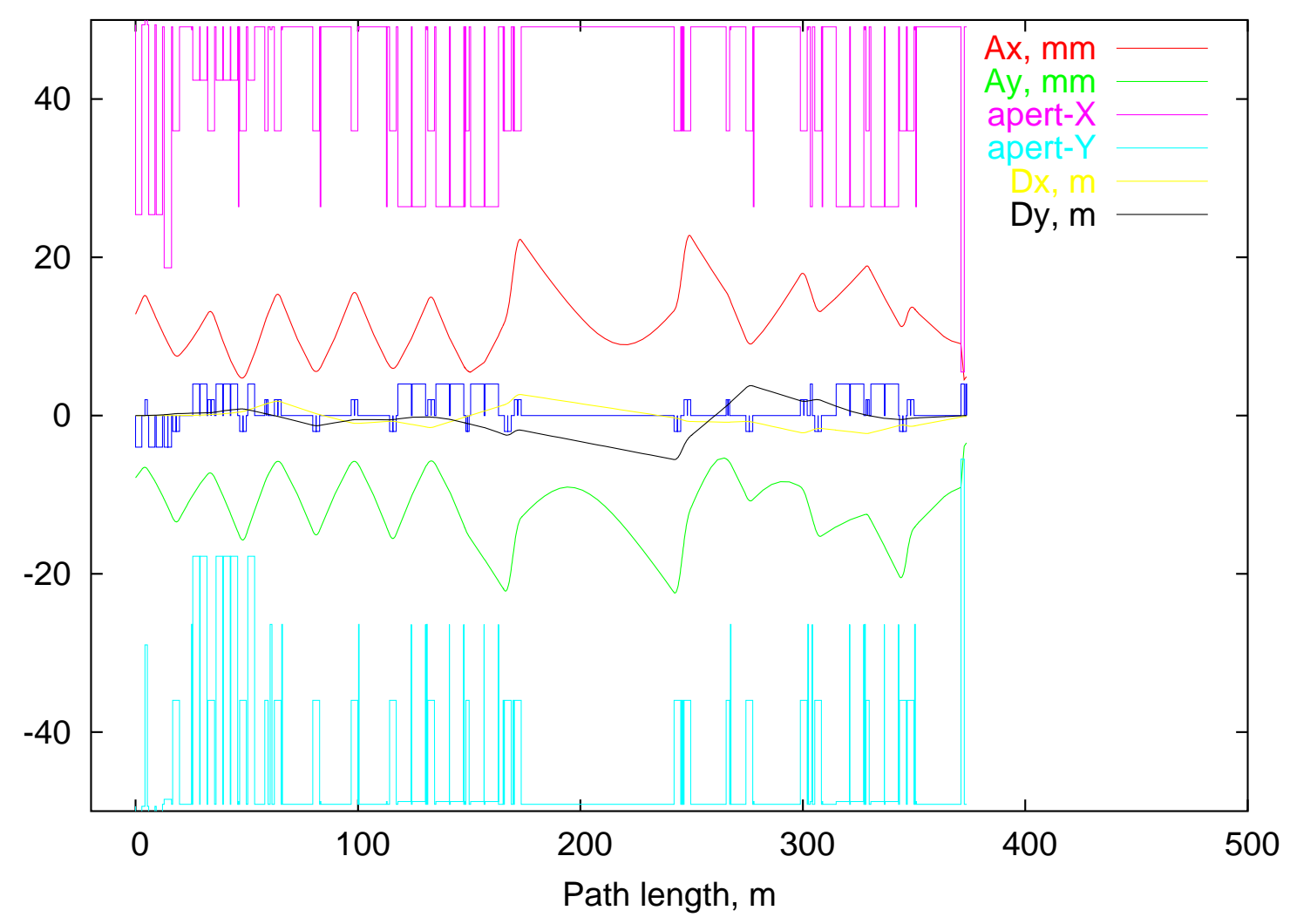

Figure 4.2: Beam size along NuMI beam-line. Maximum amplitude is $500 \pi \mathrm{mm} \mathrm{mrad}$. 

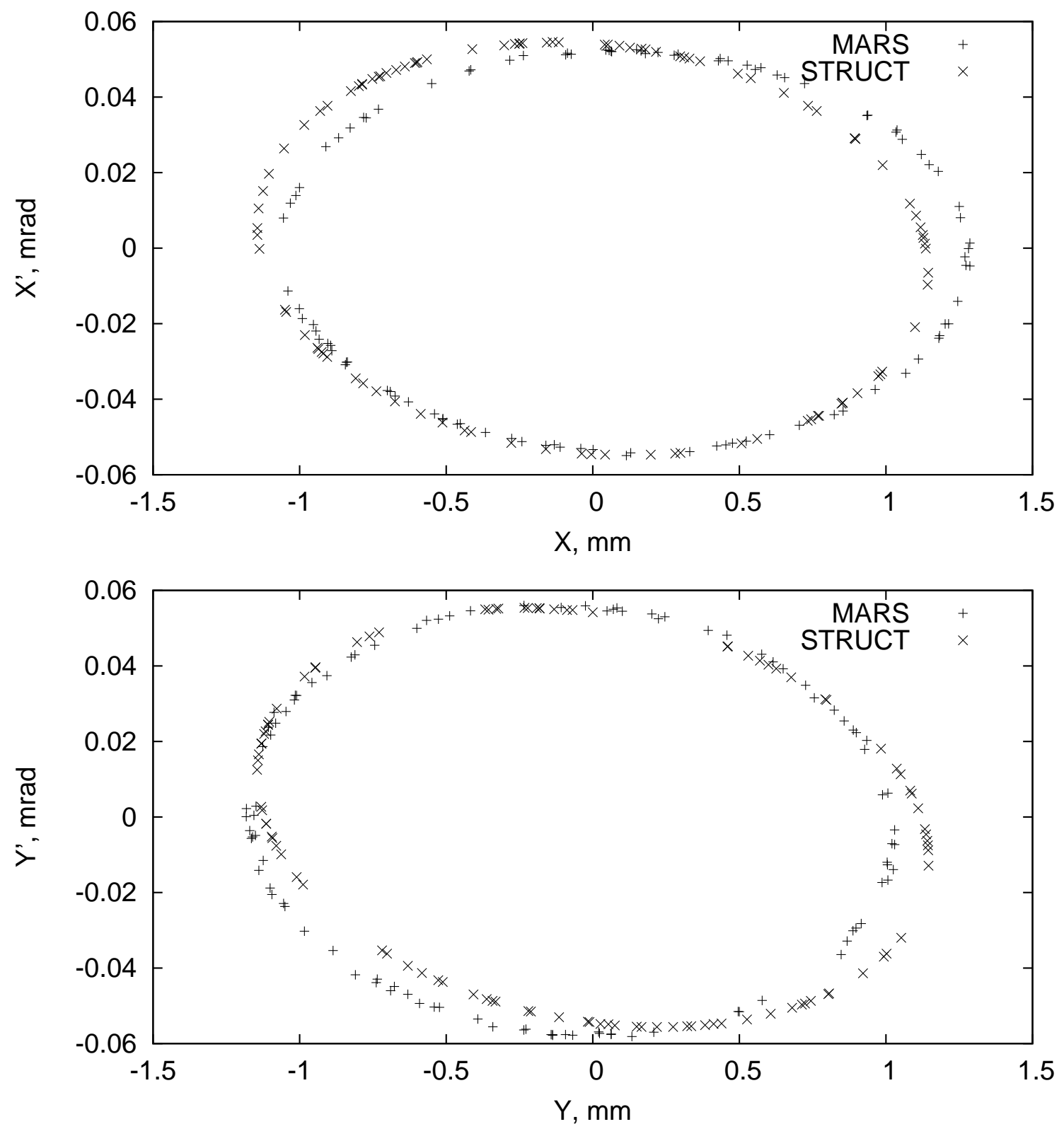

Figure 4.3: Beam distribution at BAFL2P. 


\subsection{Beam Loss vs. Magnet Current Variations}

An important consideration in the operational control of beam loss is the stability of beam positions due to NuMI power supply current variations. The power supply current regulation limits achievable for NuMI are shown in Table 4.2.

Beam losses due to current variations for individual NuMI magnets are presented in figures 4.4-6. It is seen that significant beam losses are only possible for power supply current regulation much poorer than the limits of Table 4.2. But the combined effect, when some or all magnets have deviations from nominal field, could be much larger. There are 64 possible combinations of positive-negative deviations for six magnet power supplies. For each scenario, the shift in the beam position at the end of channel (at the BAFL2P) was calculated. Maximum displacement was obtained when LAM60A, LAM61AB, V100, V118 had negative and HV101, V108 positive deviations from nominal currents. Note that if all magnets have positive (or negative) deviations the beam shift is lower by a factor of ten. These results can be simply explained -

the first four magnets move the beam up, the second two move it down. So, positive deflections in first magnets are compensated by positive deviations, or worsened by negative deflection in second pair. The dipole magnets deflect the beam horizontally only.

The kicker is not a part of the model. Using STRUCT the following relations were found between current variation $(\delta$ in $\%)$ in the kicker and the shift of horizontal coordinate $(\delta h$ in $\mathrm{cm})$ and angle $\left(\delta h^{\prime}\right.$ in $\left.\mathrm{mrad}\right)$

$$
\Delta h=3.786 \times 10^{-2} \delta
$$

and

$$
\Delta h^{\prime}=1.94 \times 10^{-2} \delta .
$$

In most cases, the accidental beam loss due to power supply regulation problems will occur in the baffle (BAFL2P), as intended. There are a few conditions, with very large deviations from normal in V108 and HV101, which can cause losses on other elements. The values for $\Delta B / B$ causing loss points other than in BAFL2P can be extracted from Fig. 4.4 and 4.5. For field variations in V108 exceeding $0.6 \%$ the beam begins to be intercepted upstream of the baffle at HT111, HQ111 and HQ113 at levels exceeding $10^{-4}$. For variations in the field of HV101 greater than $0.9 \%, 10^{-4}$ of the beam is lost on V118-2. As deviations continue to increase in HV101, the beam is lost further upstream: successively in HQ112, HT112 and finally in HQ111 (at $\Delta B / B>$ 1.5\%). These are very large deviations: 200 times the nominal allowed.

The beam loss due to current variations from $3 \times$ limits to $10 \times$ normal limits (in Table 4.2) for all magnets simultaneously was simulated using MARS and STRUCT. 
The resulting beam losses are shown in figure 4.6. It is seen that horizontal shift in both positive and negative directions due to current variation in kicker power supply gives the same beam loss.

Table.4.2 : Power supply current regulations.

\begin{tabular}{lllllll}
\hline Lam60A & Lam61AB & V100 & HV101 & V108 & V118 & kicker \\
\hline $300 p p m$ & $150 p p m$ & $150 p p m$ & $50 p p m$ & $50 p p m$ & $50 p p m$ & $0.5 \%$ \\
\hline
\end{tabular}



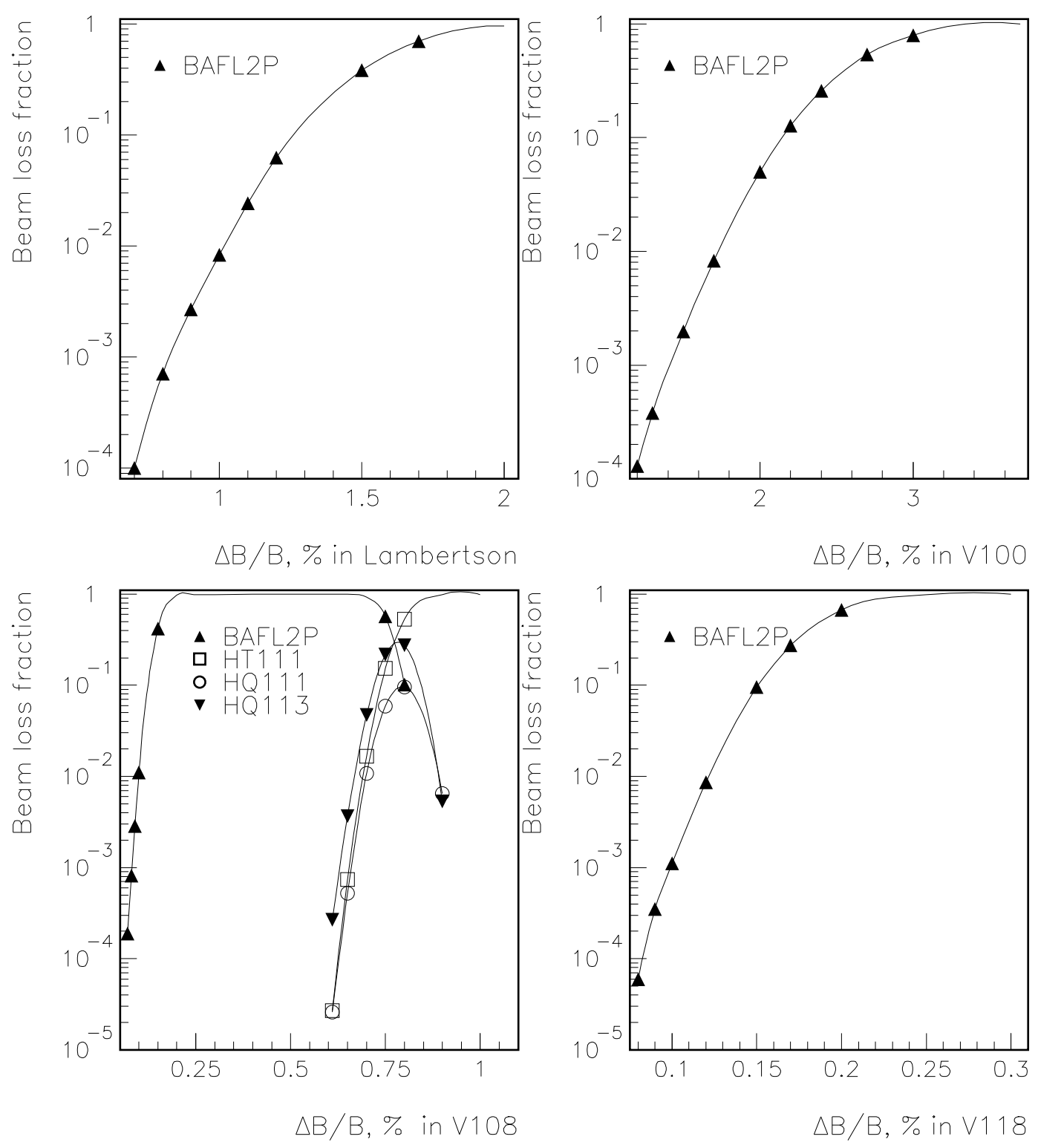

Figure 4.4: Beam loss vs. magnetic field variations. 

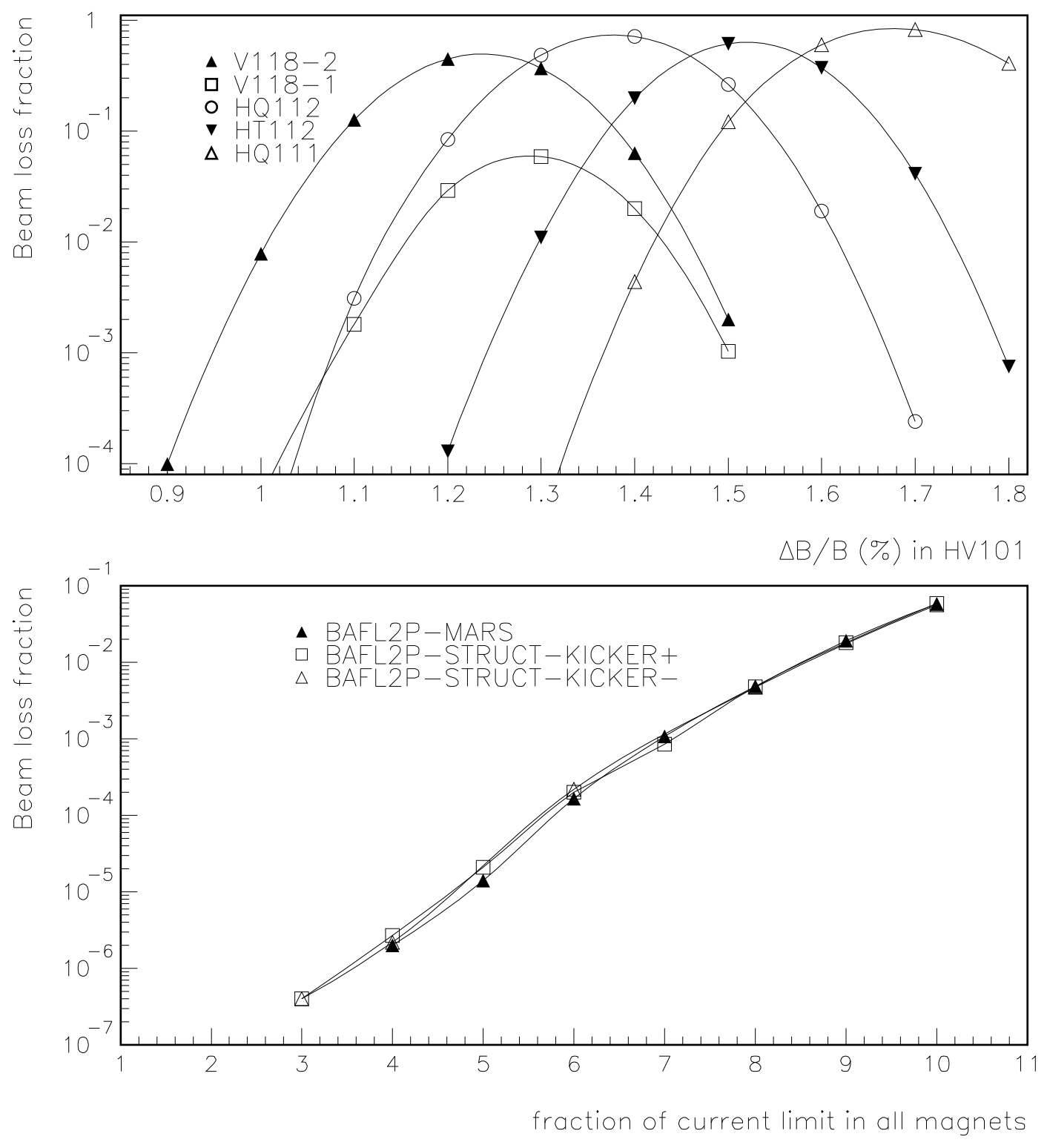

Figure 4.5: Beam loss vs. magnetic field deviation. The bottom plot represents the losses in BAFL2P as a function of the fractional deviation from the limits in Table 4.2. The loss is less than $10^{-6}$ when all magnets exceed three times their normal operating limits. The three curves show the insensitivity of the losses to the sign of the kicker magnet. 


\subsection{Star Density vs. Beam Loss Models}

Star density distributions resulting from 11 scenarios of beam loss due to magnet current variations were considered. Using results from the previous section one can find a scenario with most of the beam loss in one region. This case gives the maximum of star density for this region. A summary of beam losses for all cases are presented in table 4.3. Note that in Tables 4-3, 4-4 and 4-5 the results listed in the row labeled "wire" are inclusive: all wire scanners were IN.

Typical star density distributions for the first one centimeter of depth as a function of distance along the tunnel are shown in figures 4.6-7. Typical spatial distributions of star density are presented on figures 4.8-9. A complete set of figures for star density for all scenarios are contained in Appendix B. The maximum and average star densities in the $99.9 \%$ volume for the 7 regions and the 12 beam loss scenarios are presented in tables 4.4-5. The mean and maximal radial extent of the $99.9 \%$ volume are shown in tables 4.6-7.

Accident-condition star density limits are compared with the simulations in table 4.8. It is seen that for the worst case, the acceptable level is about 20 times greater than the star density due to the total beam loss on one magnet. The star density and losses due to beam interaction with the wire scanners will be discussed in the next section. It is important to note that star densities per lost proton are practically the same for $100 \%$ and $0.01 \%$ beam loss. 
Table.4.3 : Worst case beam loss (particle/proton) for the regions. Note that the largest potential losses are from directly hitting HQ111 and HQ112

\begin{tabular}{llllllll}
\hline source/region & HQ111 & HQ112 & 3 & 4 & 5 & 6 & 7 \\
\hline HV101 1.20\% & - & 0.1505 & - & 0.3929 & 0.2265 & - & - \\
\hline HV101 1.25\% & - & 0.3552 & - & 0.4100 & 0.1401 & - & - \\
\hline HV101 1.40\% & 0.005 & 0.944 & - & 0.045 & 0.004 & - & - \\
\hline HV101 1.50\% & 0.1141 & 0.7635 & - & 0.002 & - & - & - \\
\hline V108 0.75\% & 0.2678 & - & 0.0775 & - & - & 0.03 & 0.6032 \\
\hline V108 0.78\% & 0.5144 & - & 0.1262 & - & & 0.0542 & 0.3040 \\
\hline V108 0.85\% & 0.9442 & - & 0.0485 & - & & 0.038 & 0.0029 \\
\hline V108 0.90\% & 0.9921 & - & 0.035 & - & & 0.001 & - \\
\hline comb1 & - & 0.902 & 0.0015 & 0.0908 & 0.0038 & - & - \\
\hline comb2 & 0.0087 & - & 0.9912 & - & - & - & 0.0001 \\
\hline comb3 & 0.0474 & - & 0.0116 & - & - & 0.941 & - \\
\hline wire & $8.3 \times 10^{-5}$ & $7.2 \times 10^{-6}$ & $2.2 \times 10^{-5}$ & $8.3 \times 10^{-5}$ & - & - & $7.2 \times 10^{-6}$ \\
\hline
\end{tabular}

comb1 - HV101 1.4\% @ V108 0.1\%

comb2 - HV101 0.5\% @ V108 0.8\%

comb3 - V118 1.0\%@ @108 0.7\%

wire - all wire scanners IN

Table.4.4 : Star density (star/cc/pot) for the worst-case beam loss.

\begin{tabular}{llllllll}
\hline source/region & 1 & 2 & 3 & 4 & 5 & 6 & 7 \\
\hline HV101 1.20\% & $5.0 \times 10^{-8}$ & $1.3 \times 10^{-8}$ & $2.8 \times 10^{-7}$ & $6.5 \times 10^{-7}$ & $8.0 \times 10^{-8}$ & $7.9 \times 10^{-9}$ & $6.9 \times 10^{-9}$ \\
\hline HV101 $1.25 \%$ & $1.3 \times 10^{-7}$ & $2.8 \times 10^{-8}$ & $2.9 \times 10^{-7}$ & $9.5 \times 10^{-7}$ & $5.6 \times 10^{-8}$ & $1.1 \times 10^{-8}$ & $4.9 \times 10^{-9}$ \\
\hline HV101 $1.40 \%$ & $1.8 \times 10^{-7}$ & $3.7 \times 10^{-8}$ & $5.7 \times 10^{-8}$ & $1.2 \times 10^{-7}$ & $6.7 \times 10^{-9}$ & $3.6 \times 10^{-9}$ & $1.7 \times 10^{-9}$ \\
\hline HV101 1.50\% & $2.2 \times 10^{-7}$ & $4.6 \times 10^{-8}$ & $1.9 \times 10^{-7}$ & $6.5 \times 10^{-8}$ & $7.8 \times 10^{-9}$ & $2.3 \times 10^{-9}$ & $2.0 \times 10^{-9}$ \\
\hline V108 0.75\% & $3.8 \times 10^{-8}$ & $1.0 \times 10^{-8}$ & $2.0 \times 10^{-7}$ & $2.7 \times 10^{-9}$ & $3.2 \times 10^{-9}$ & $7.8 \times 10^{-9}$ & $1.4 \times 10^{-7}$ \\
\hline V108 0.78\% & $8.8 \times 10^{-8}$ & $1.0 \times 10^{-8}$ & $4.0 \times 10^{-7}$ & $4.4 \times 10^{-9}$ & $5.7 \times 10^{-9}$ & $8.9 \times 10^{-9}$ & $7.3 \times 10^{-8}$ \\
\hline V108 0.85\% & $2.5 \times 10^{-7}$ & $3.6 \times 10^{-8}$ & $2.7 \times 10^{-7}$ & $4.4 \times 10^{-9}$ & $3.0 \times 10^{-9}$ & $7.2 \times 10^{-9}$ & $2.7 \times 10^{-9}$ \\
\hline V108 0.90\% & $2.5 \times 10^{-7}$ & $5.7 \times 10^{-8}$ & $2.9 \times 10^{-7}$ & $9.8 \times 10^{-9}$ & $4.7 \times 10^{-9}$ & $1.9 \times 10^{-8}$ & $5.5 \times 10^{-9}$ \\
\hline comb 1 & $2.1 \times 10^{-7}$ & $5.2 \times 10^{-8}$ & $7.3 \times 10^{-8}$ & $1.8 \times 10^{-8}$ & $1.1 \times 10^{-8}$ & $2.8 \times 10^{-9}$ & $2.4 \times 10^{-9}$ \\
\hline comb 2 & $2.3 \times 10^{-9}$ & $1.1 \times 10^{-9}$ & $3.8 \times 10^{-6}$ & $3.6 \times 10^{-8}$ & $6.0 \times 10^{-9}$ & $4.5 \times 10^{-9}$ & $2.2 \times 10^{-9}$ \\
\hline comb 3 & $5.7 \times 10^{-9}$ & $1.9 \times 10^{-9}$ & $2.6 \times 10^{-8}$ & $5.9 \times 10^{-10}$ & $5.2 \times 10^{-8}$ & $2.5 \times 10^{-7}$ & $5.5 \times 10^{-8}$ \\
\hline wire & $1.1 \times 10^{-11}$ & $3.2 \times 10^{-12}$ & $2.9 \times 10^{-11}$ & $1.4 \times 10^{-10}$ & $3.1 \times 10^{-11}$ & $7.2 \times 10^{-12}$ & $2.6 \times 10^{-12}$ \\
\hline limit & $1 \times 10^{-4}$ & $1 \times 10^{-6}$ & $1 \times 10^{-4}$ & $1 \times 10^{-4}$ & $1 \times 10^{-4}$ & $1 \times 10^{-4}$ & $1 \times 10^{-4}$ \\
\hline
\end{tabular}


Table.4.5 : Worst-case beam loss average star density (star/cc/pot) in $99.9 \%$ volume.

\begin{tabular}{llllllll}
\hline source/region & 1 & 2 & 3 & 4 & 5 & 6 & 7 \\
\hline HV101 1.20\% & $3.4 \times 10^{-9}$ & $1.1 \times 10^{-9}$ & $3.9 \times 10^{-9}$ & $3.2 \times 10^{-8}$ & $6.0 \times 10^{-9}$ & $6.9 \times 10^{-10}$ & $3.4 \times 10^{-10}$ \\
\hline HV101 1.25\% & $7.4 \times 10^{-9}$ & $2.3 \times 10^{-9}$ & $5.9 \times 10^{-9}$ & $3.1 \times 10^{-8}$ & $3.9 \times 10^{-9}$ & $5.4 \times 10^{-10}$ & $2.8 \times 10^{-10}$ \\
\hline HV101 1.40\% & $1.3 \times 10^{-8}$ & $3.8 \times 10^{-9}$ & $6.7 \times 10^{-9}$ & $4.0 \times 10^{-9}$ & $4.8 \times 10^{-10}$ & $1.8 \times 10^{-10}$ & $9.1 \times 10^{-11}$ \\
\hline HV101 1.50\% & $1.3 \times 10^{-8}$ & $4.4 \times 10^{-9}$ & $1.3 \times 10^{-8}$ & $3.5 \times 10^{-9}$ & $5.3 \times 10^{-10}$ & $1.8 \times 10^{-10}$ & $8.2 \times 10^{-11}$ \\
\hline V108 0.75\% & $2.5 \times 10^{-9}$ & $5.2 \times 10^{-10}$ & $6.8 \times 10^{-9}$ & $8.8 \times 10^{-11}$ & $1.0 \times 10^{-10}$ & $7.0 \times 10^{-10}$ & $9.2 \times 10^{-9}$ \\
\hline V108 0.78\% & $5.2 \times 10^{-9}$ & $9.1 \times 10^{-10}$ & $1.3 \times 10^{-8}$ & $1.4 \times 10^{-10}$ & $1.3 \times 10^{-10}$ & $6.8 \times 10^{-10}$ & $5.0 \times 10^{-9}$ \\
\hline V108 0.85\% & $1.5 \times 10^{-8}$ & $3.4 \times 10^{-9}$ & $1.3 \times 10^{-8}$ & $2.5 \times 10^{-10}$ & $1.6 \times 10^{-10}$ & $6.2 \times 10^{-10}$ & $1.7 \times 10^{-10}$ \\
\hline V108 0.90\% & $1.7 \times 10^{-8}$ & $6.5 \times 10^{-9}$ & $1.9 \times 10^{-8}$ & $4.7 \times 10^{-10}$ & $2.7 \times 10^{-9}$ & $1.5 \times 10^{-9}$ & $3.0 \times 10^{-10}$ \\
\hline comb 1 & $1.4 \times 10^{-8}$ & $4.4 \times 10^{-9}$ & $7.1 \times 10^{-9}$ & $9.0 \times 10^{-9}$ & $6.8 \times 10^{-10}$ & $2.4 \times 10^{-10}$ & $1.2 \times 10^{-10}$ \\
\hline comb 2 & $1.0 \times 10^{-10}$ & $3.7 \times 10^{-11}$ & $1.3 \times 10^{-7}$ & $3.2 \times 10^{-9}$ & $3.5 \times 10^{-10}$ & $1.9 \times 10^{-10}$ & $1.3 \times 10^{-10}$ \\
\hline comb 3 & $3.7 \times 10^{-10}$ & $1.1 \times 10^{-10}$ & $9.2 \times 10^{-10}$ & $1.5 \times 10^{-11}$ & $2.3 \times 10^{-9}$ & $1.9 \times 10^{-8}$ & $2.6 \times 10^{-9}$ \\
\hline wire & $1.7 \times 10^{-12}$ & $3.8 \times 10^{-13}$ & $3.3 \times 10^{-12}$ & $6.4 \times 10^{-12}$ & $1.2 \times 10^{-12}$ & $3.1 \times 10^{-13}$ & $1.6 \times 10^{-13}$ \\
\hline
\end{tabular}

Table.4.6 : Star-density-weighted mean radial thickness $(\mathrm{cm})$

\begin{tabular}{llllllll}
\hline source/region & 1 & 2 & 3 & 4 & 5 & 6 & 7 \\
\hline HV101 1.20\% & 56 & 55 & 50 & 28 & 31 & 30 & 31 \\
\hline HV101 1.25\% & 57 & 55 & 50 & 36 & 37 & 32 & 37 \\
\hline HV101 1.40\% & 60 & 55 & 50 & 36 & 36 & 31 & 40 \\
\hline HV101 1.50\% & 62 & 53 & 50 & 27 & 29 & 30 & 33 \\
\hline V108 0.75\% & 62 & 51 & 53 & 29 & 77 & 30 & 28 \\
\hline V108 0.78\% & 67 & 52 & 52 & 27 & 49 & 32 & 29 \\
\hline V108 0.85\% & 65 & 51 & 49 & 28 & 33 & 32 & 25 \\
\hline V108 0.90\% & 63 & 51 & 49 & 26 & 35 & 32 & 38 \\
\hline comb 1 & 59 & 55 & 49 & 28 & 29 & 29 & 31 \\
\hline comb 2 & 67 & 52 & 50 & 28 & 29 & 32 & 33 \\
\hline comb 3 & 63 & 52 & 51 & 71 & 63 & 35 & 51 \\
\hline wire & 72 & 52 & 47 & 36 & 33 & 30 & 35 \\
\hline
\end{tabular}


Table.4.7 : Radial thickness (cm) of the $99.9 \%$ volume.

\begin{tabular}{llllllll}
\hline source/region & 1 & 2 & 3 & 4 & 5 & 6 & 7 \\
\hline HV101 1.20\% & 428 & 278 & 188 & 195 & 195 & 195 & 285 \\
\hline HV101 1.25\% & 428 & 293 & 203 & 165 & 225 & 165 & 285 \\
\hline HV101 1.40\% & 503 & 323 & 233 & 195 & 255 & 165 & 285 \\
\hline HV101 1.50\% & 488 & 378 & 233 & 195 & 165 & 195 & 285 \\
\hline V108 0.75\% & 518 & 248 & 248 & 195 & 285 & 195 & 195 \\
\hline V108 0.78\% & 503 & 293 & 248 & 195 & 285 & 225 & 165 \\
\hline V108 0.85\% & 488 & 263 & 233 & 195 & 285 & 195 & 285 \\
\hline V108 0.90\% & 488 & 233 & 233 & 195 & 285 & 195 & 285 \\
\hline comb 1 & 458 & 308 & 248 & 165 & 255 & 225 & 285 \\
\hline comb 2 & 458 & 248 & 333 & 195 & 255 & 195 & 285 \\
\hline comb 3 & 563 & 353 & 233 & 285 & 285 & 195 & 285 \\
\hline wire & 503 & 383 & 248 & 195 & 195 & 135 & 285 \\
\hline
\end{tabular}

Table.4.8 : Accidental beam loss.

\begin{tabular}{lllll}
\hline region & $\begin{array}{l}\text { star density limit } \\
\text { star } / \text { cc/proton }\end{array}$ & $\begin{array}{l}\text { maximum } \\
\text { star/cc/lost proton }\end{array}$ & origin & $\begin{array}{l}\text { wire scanners } \\
\text { star/cc/lost proton }\end{array}$ \\
\hline 1 & $5.3 \times 10^{3}$ & $1.7 \times 10^{-8}$ & $99 \%$ loss on HT111 & $1.8 \times 10^{-8}$ \\
2 & $2.8 \times 10^{-6}$ & $6.6 \times 10^{-9}$ & $99 \%$ loss on HT111 & $4.2 \times 10^{-9}$ \\
3 & $2.7 \times 10^{-6}$ & $1.3 \times 10^{-7}$ & $99 \%$ loss on HQ113 & $1.5 \times 10^{-7}$ \\
4 & $2.7 \times 10^{-6}$ & $8.2 \times 10^{-8}$ & $39 \%$ loss on HT117 & $7.7 \times 10^{-8}$ \\
5 & $2.7 \times 10^{-6}$ & $2.6 \times 10^{-8}$ & $23 \%$ loss on V118-2 & \\
6 & $2.7 \times 10^{-6}$ & $2.0 \times 10^{-8}$ & $94 \%$ loss on V118-4 & \\
7 & $2.7 \times 10^{-6}$ & $1.5 \times 10^{-8}$ & $60 \%$ loss on HT121 & $2.2 \times 10^{-8}$ \\
\hline
\end{tabular}



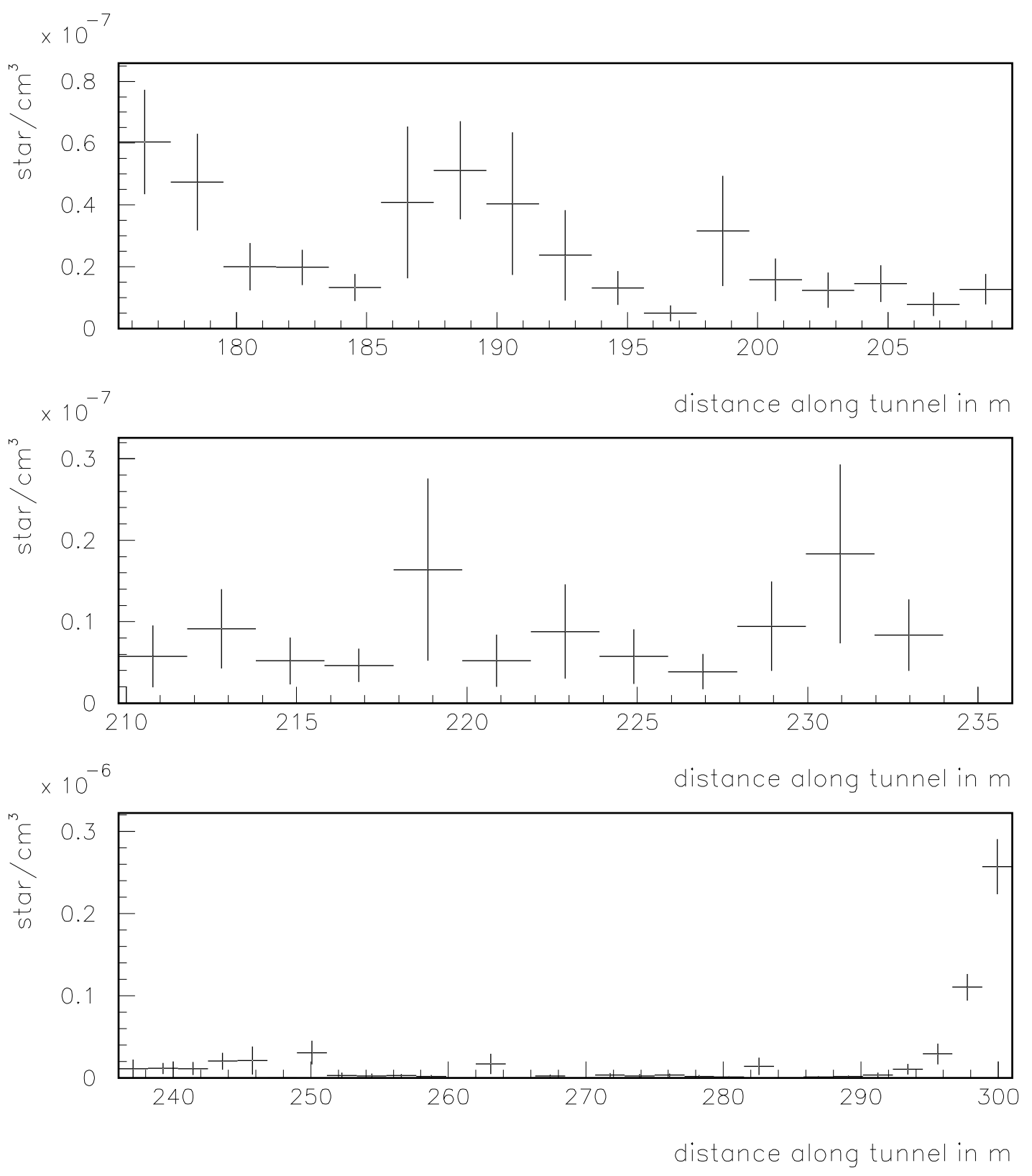

Star density distribution in $1 \mathrm{~cm}$ bin.

Figure 4.6: Star density distribution for first three regions. Magnet current variation on $1.2 \%$ in HV101. The top plot begins at the gate just downstream of Q112. The bottom plot ends at the Pre-target gate. 

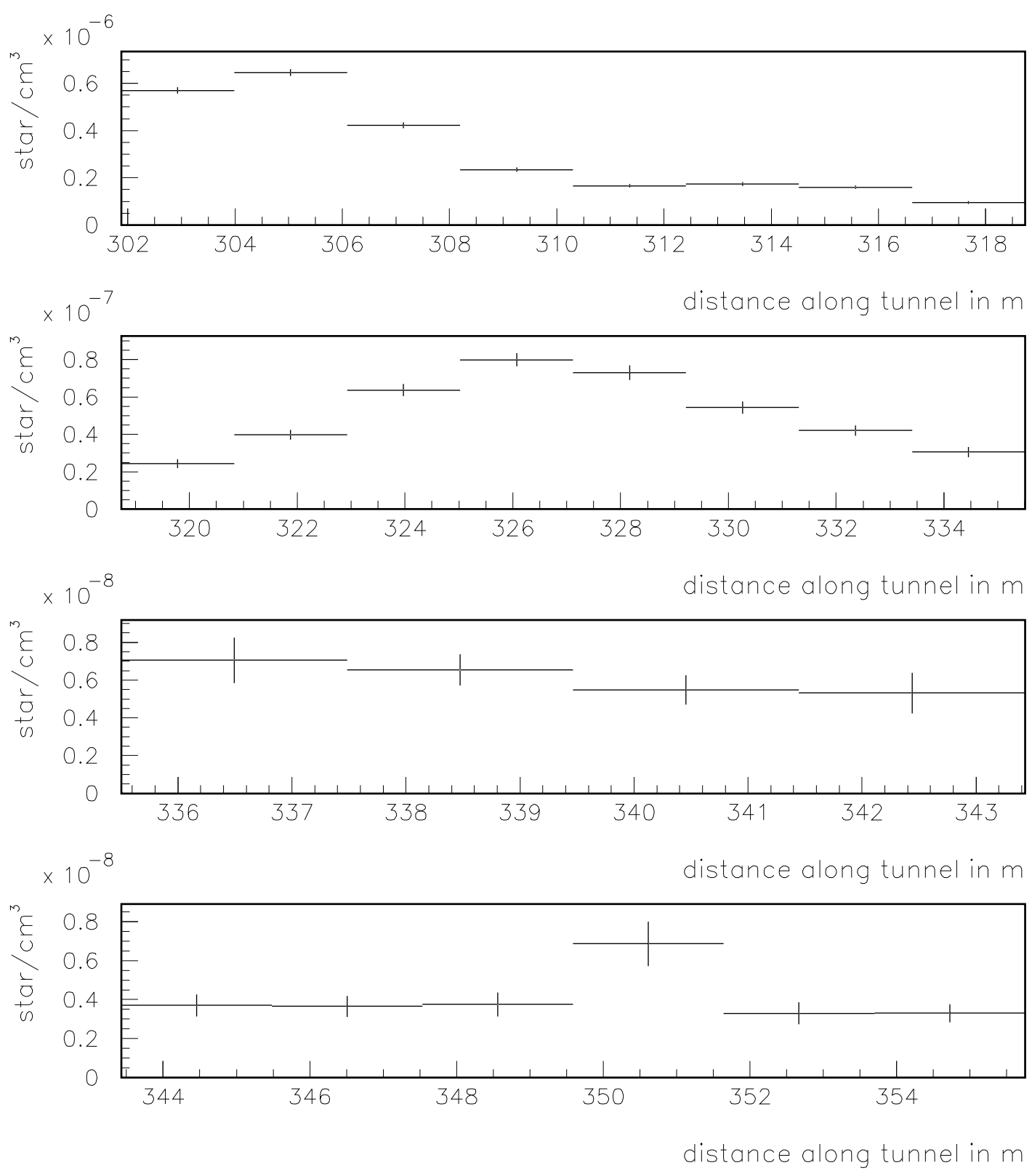

Star density distribution in $1 \mathrm{~cm}$ bin.

Figure 4.7: Star density distribution for last four regions. Magnet current variation on $1.2 \%$ in HV101. The top plot begins at the Pre-target gate. 

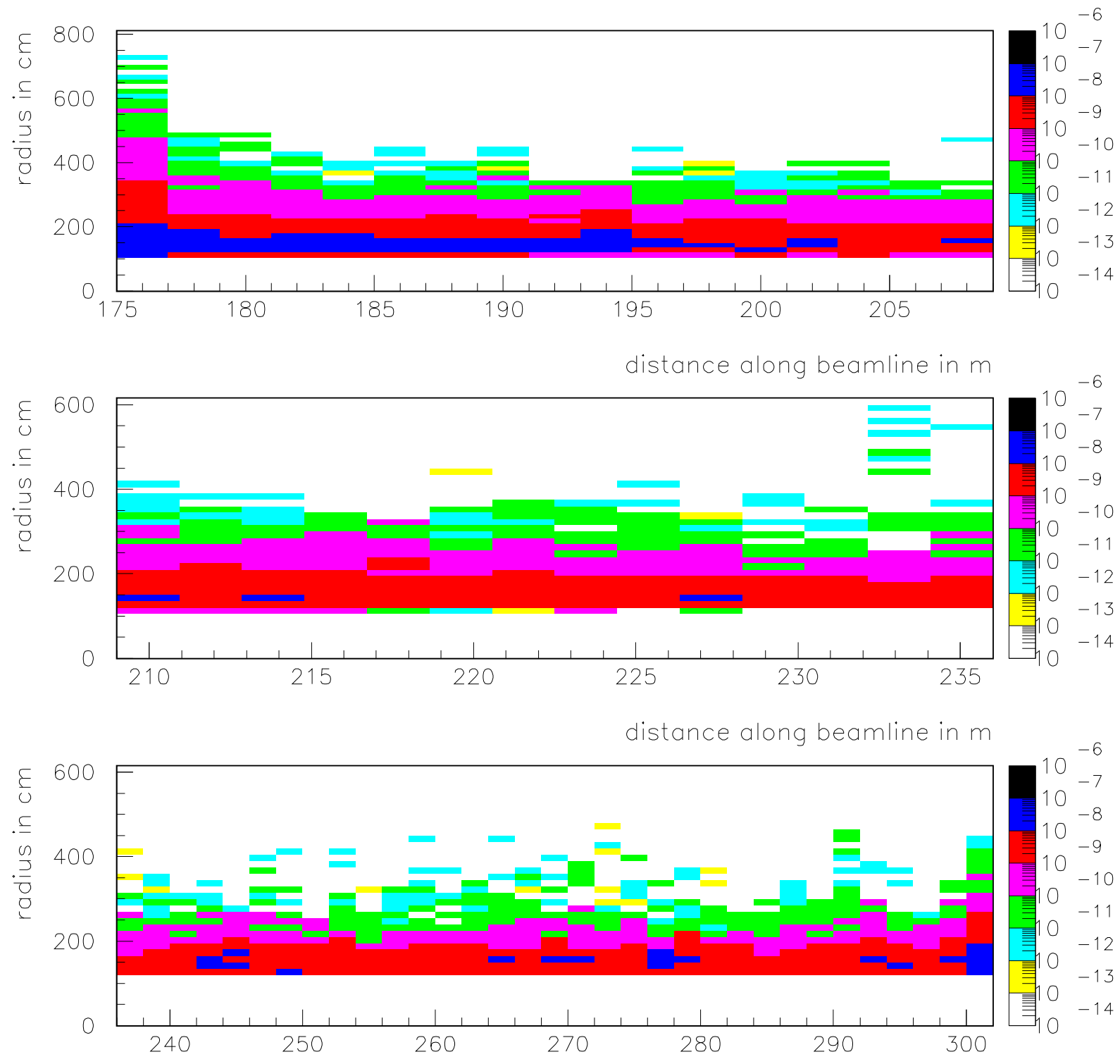

distance along beamline in $m$

Star density distribution. 1.2\% magnetic field variation in HV101

Figure 4.8: Star density distribution for first three regions. The top plot begins at the gate just downstream of Q112. The bottom plot ends at the Pre-target gate. 

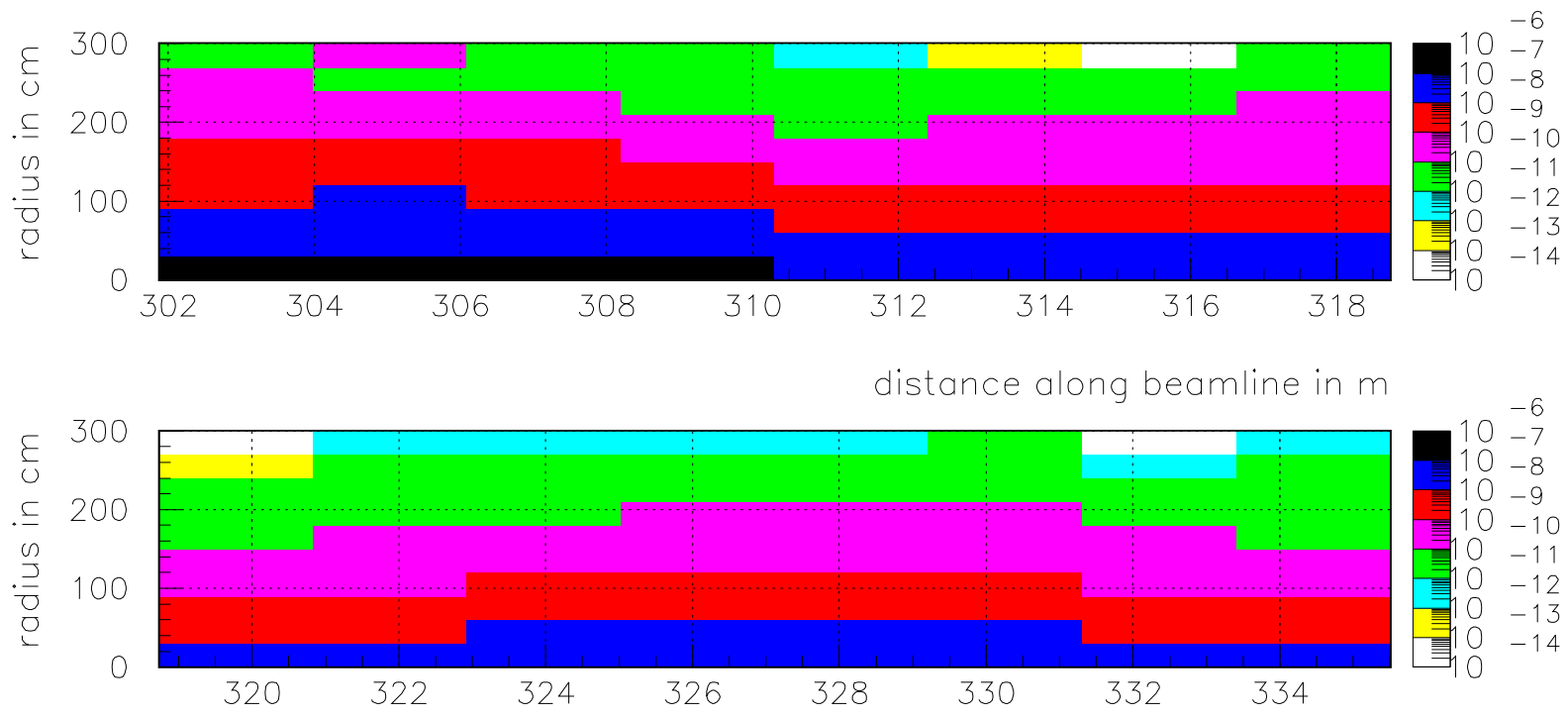

distance along beamline in $\mathrm{m}$

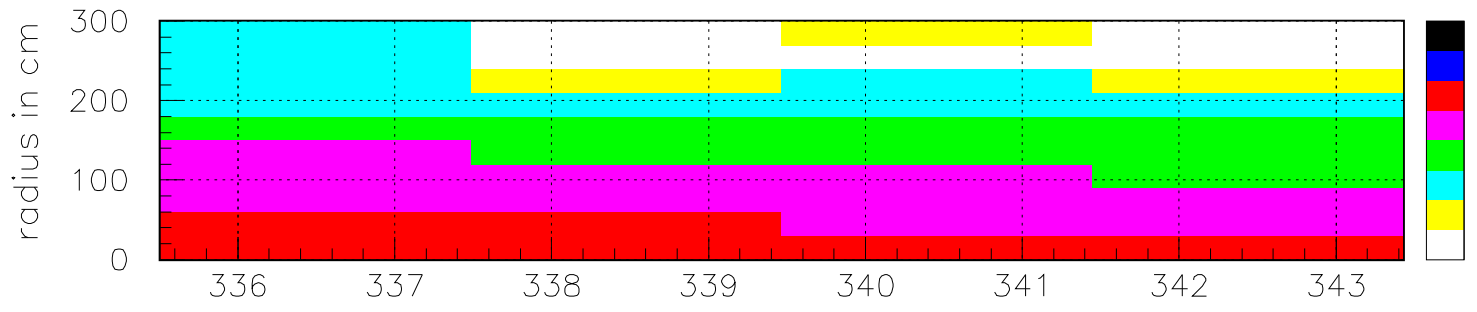

distance along beamline in $m$

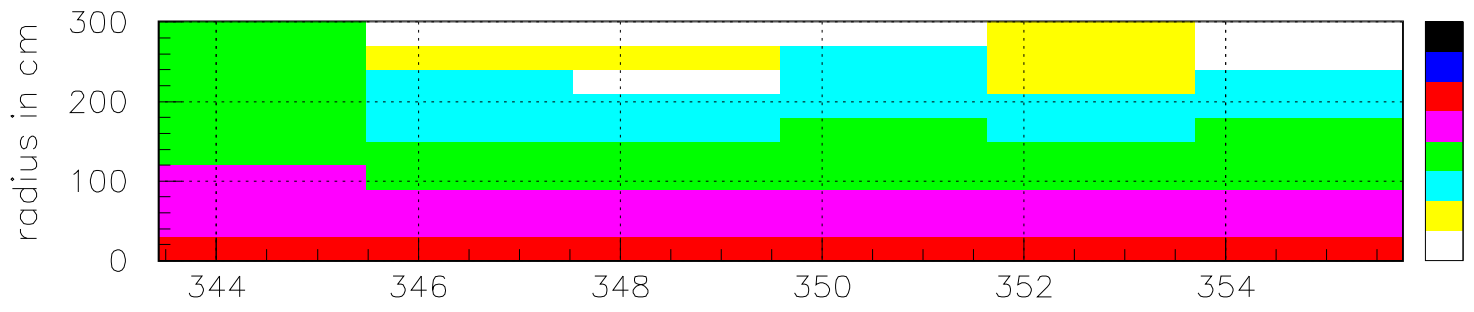

distance along beamline in $m$

Star density distribution. 1.2\% magnetic field variation in HV101

Figure 4.9: Star density distribution for last four regions. The top plot begins at the Pre-target gate. 


\subsection{Multi-wire Beam Loss}

Two types of wire scanners are to be used in the NuMI channel. The first consists of two planes of tungsten wires, each wire 75 microns in diameter. The separation between wires is $1 \mathrm{~mm}$. The probability of a primary proton interacting with this device is $8.3 \times 10^{-5}$. There are only two such scanners in the channel - PM111 and PM118.

All the other multi-wires are much thinner and use strips instead of wires. Each strip thickness is 5 microns of titanium, and the width is $0.25 \mathrm{~mm}$. The strips are separated by $1 \mathrm{~mm}$. Again each device has two planes of strips. The probability of interactions with this scanner is ten times smaller: $8.3 \times 10^{-6}$.

In the Tables and Figures that follow, the results are calculated with all wire scanners IN, simultaneously. Star densities due to beam interactions with wire scanners are shown in figures 4.10-4.13. A summary of beam losses is presented in Table 4.3. The average star density in the $99.9 \%$ volume, and the average and maximum radius of this volume are shown in Tables 4.4-4.7.

Star densities due to beam interaction with all wire scanners IN are compared with operational limits in Table 4.9. The effects of losses on the wire scanners is within the acceptable limits.

Residual activity due to interaction of beam protons with wire scanners is presented in figure 4.14. It is important to note that the data shown in fig. 4.14 represents the residual dose on contact for the various beamline elements assuming a continuous 30day irradiation at full beam intensity with all wirescanner IN simultaneously. While this mode will not be part of NuMI running conditions, for the purposes of this study it represents a worst-case scenario. Even so, residual dose on contact is within accepted limits. The neutron flux and prompt dose, averaged over the surface of the magnet facing the wire scanners, is also given in tabular form in Table 4.10. Finally, the calculated losses for each wire chamber are given in Table 4.11.

Table.4.9 : Operational beam loss, star/cc/proton. All wire scanners are IN.

\begin{tabular}{lll}
\hline region & star density limit & star density - wire \\
\hline 1 & $6.4 \times 10^{-4}$ & $1.7 \times 10^{-12}$ \\
2 & $2.1 \times 10^{-12}$ & $3.8 \times 10^{-13}$ \\
3 & $5.7 \times 10^{-10}$ & $3.3 \times 10^{-12}$ \\
4 & $4.8 \times 10^{-10}$ & $6.4 \times 10^{-12}$ \\
5 & $6.4 \times 10^{-10}$ & $1.2 \times 10^{-12}$ \\
6 & $6.4 \times 10^{-10}$ & $3.1 \times 10^{-13}$ \\
7 & $6.4 \times 10^{-10}$ & $1.6 \times 10^{-13}$ \\
\hline
\end{tabular}



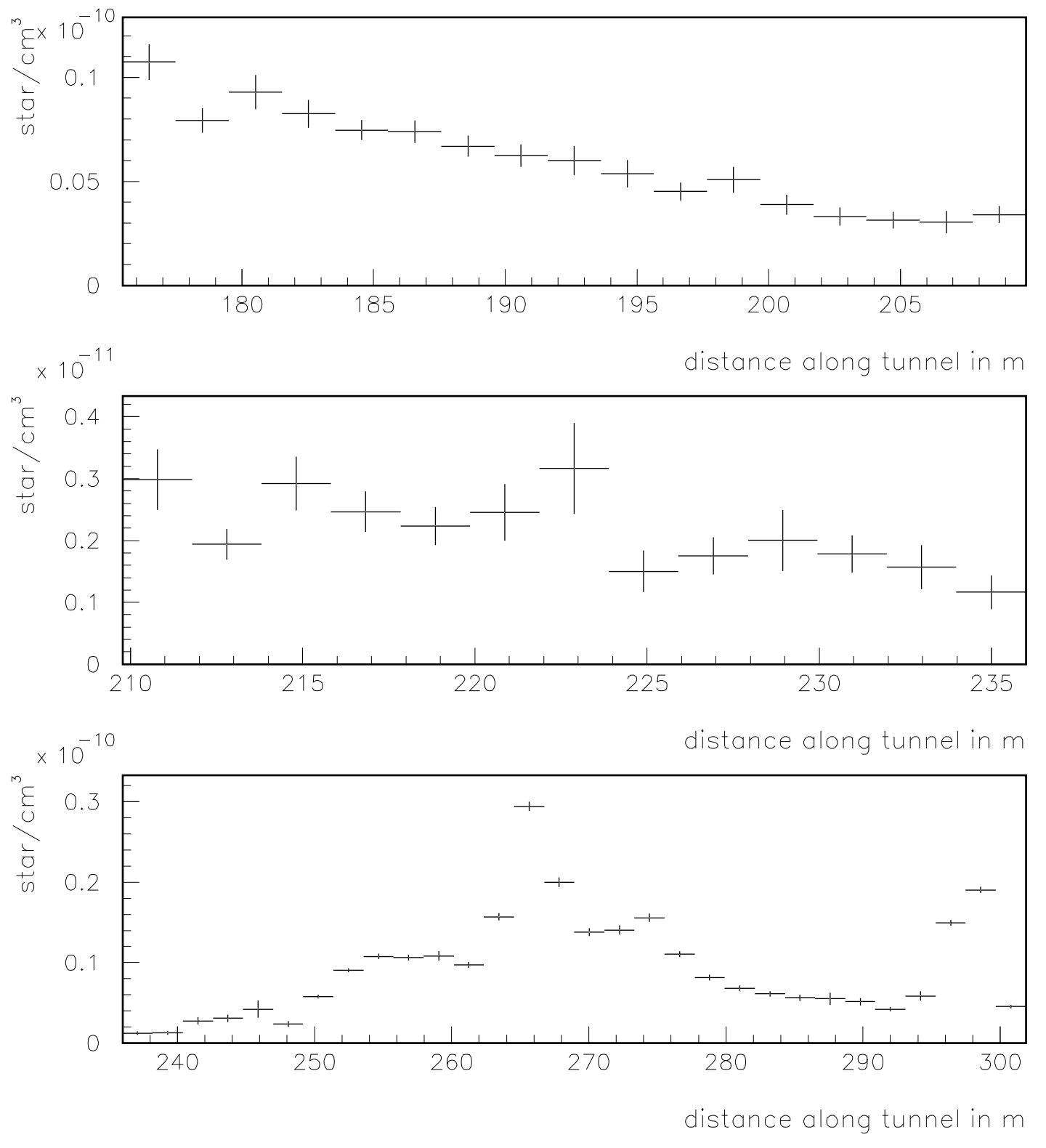

Star density distribution in $1 \mathrm{~cm}$ bin.

Figure 4.10: Star density distribution for first three regions. Wire scanners. The top plot begins at the gate just downstream of Q112. The bottom plot ends at the Pre-target gate. 

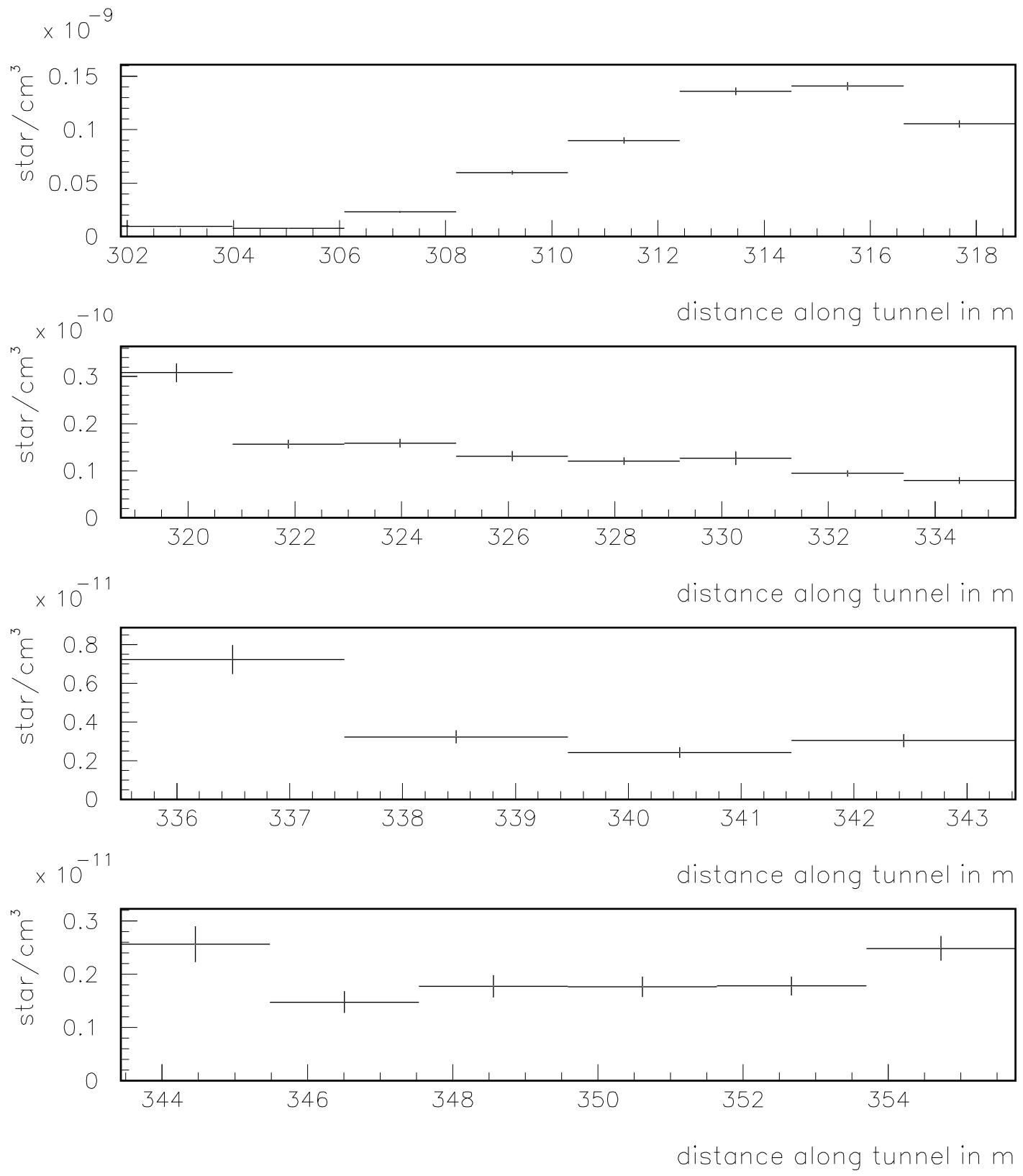

Star density distribution in $1 \mathrm{~cm}$ bin.

Figure 4.11: Star density distribution for last four regions. Wire scanners. The top plot begins at the Pre-target gate. 

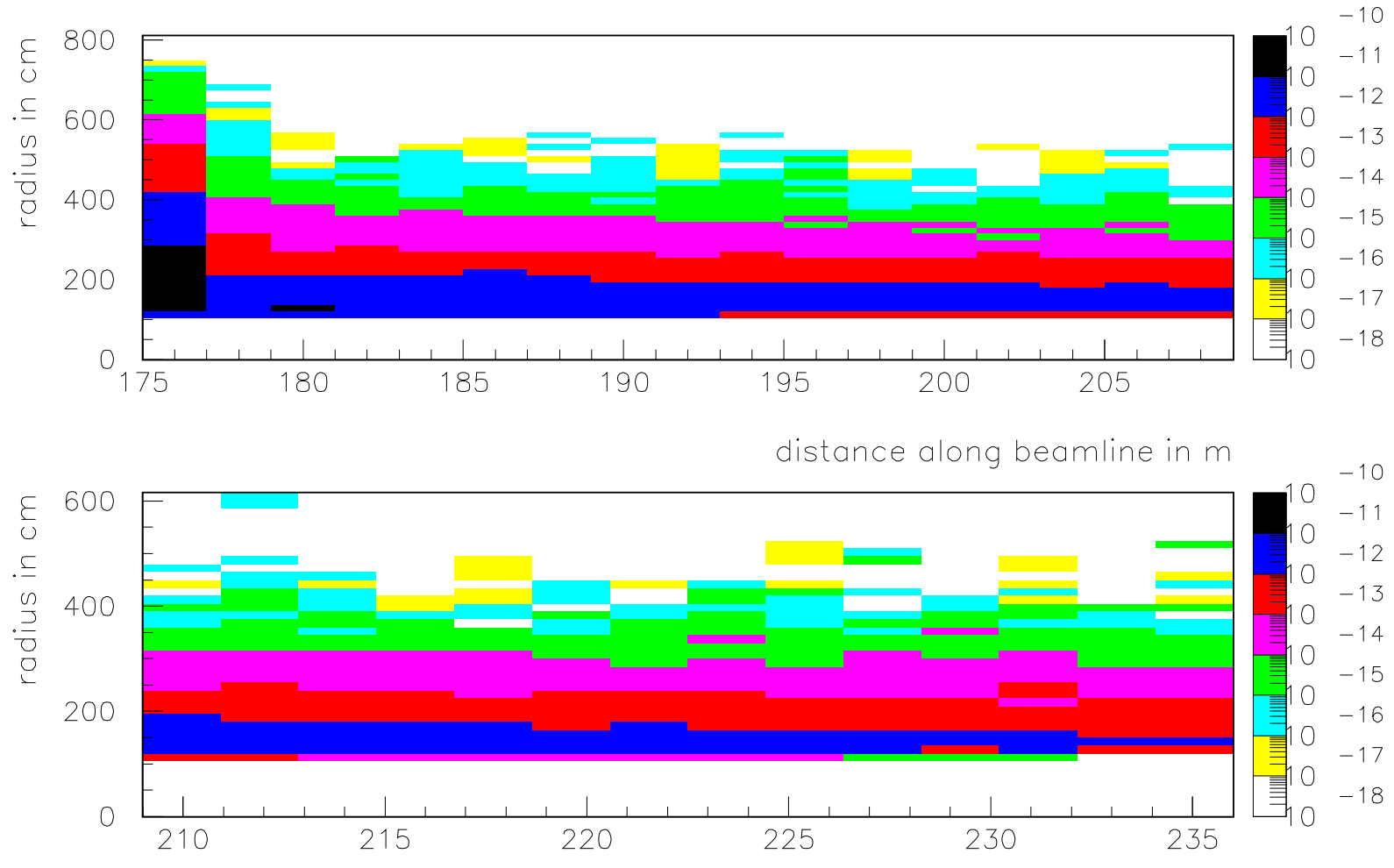

distance along beamline in $\mathrm{m}$

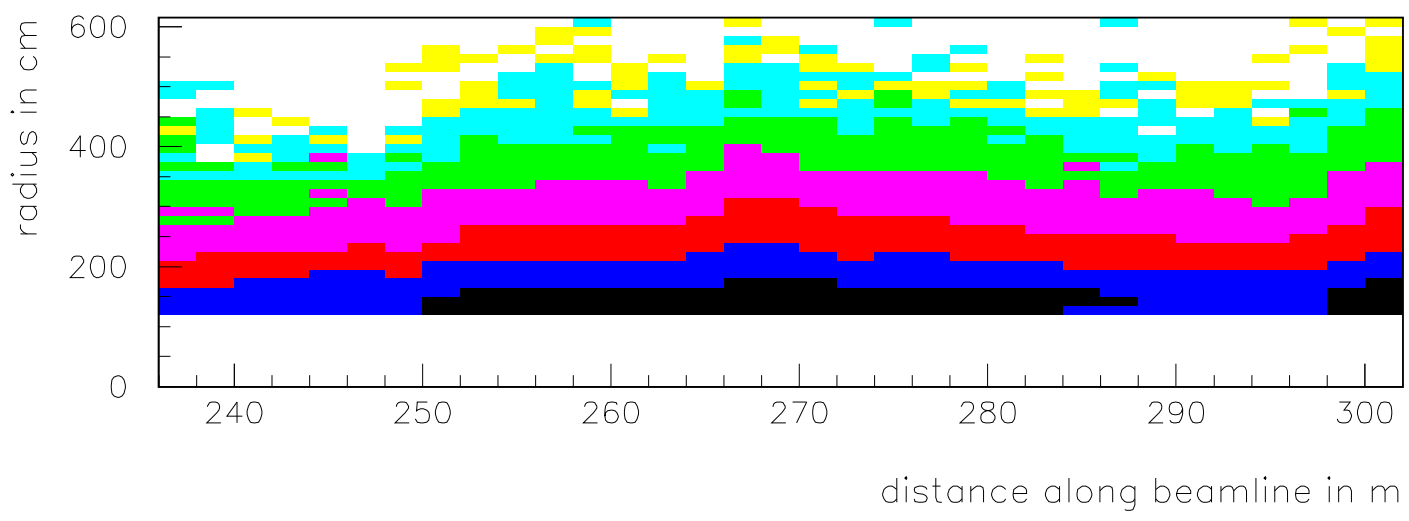

Star density. Wire scanner.

Figure 4.12: Star density distribution for first three regions. The top plot begins at the gate just downstream of Q112. The bottom plot ends at the Pre-target gate. 

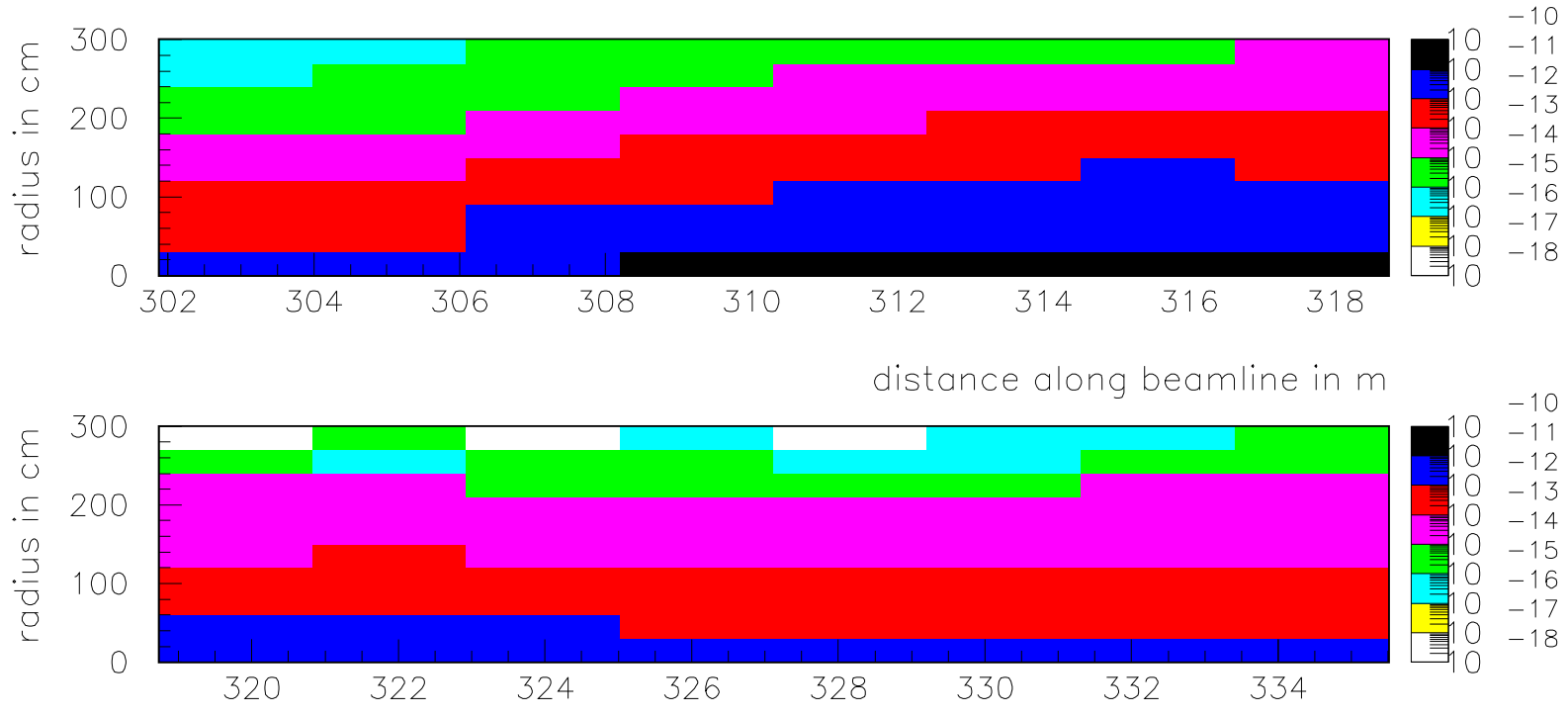

distance along beamline in $\mathrm{m}$

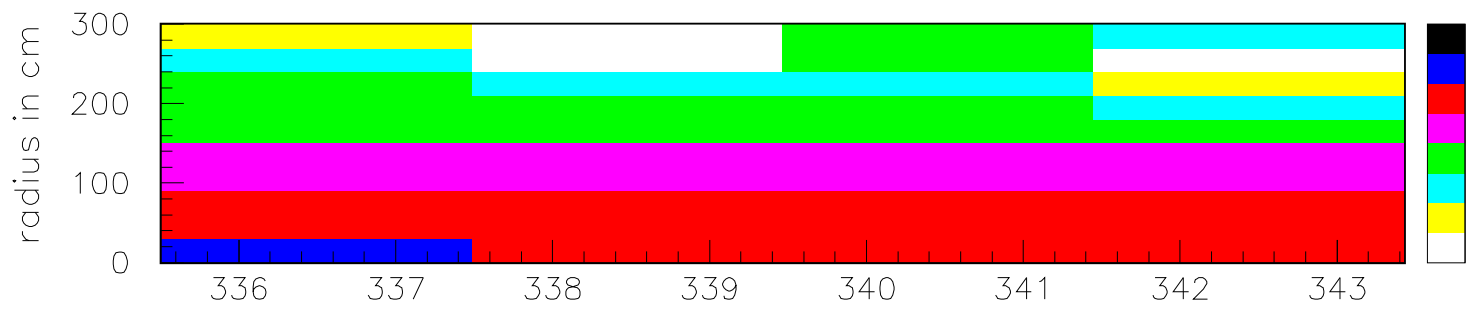

distance along beamline in $m$

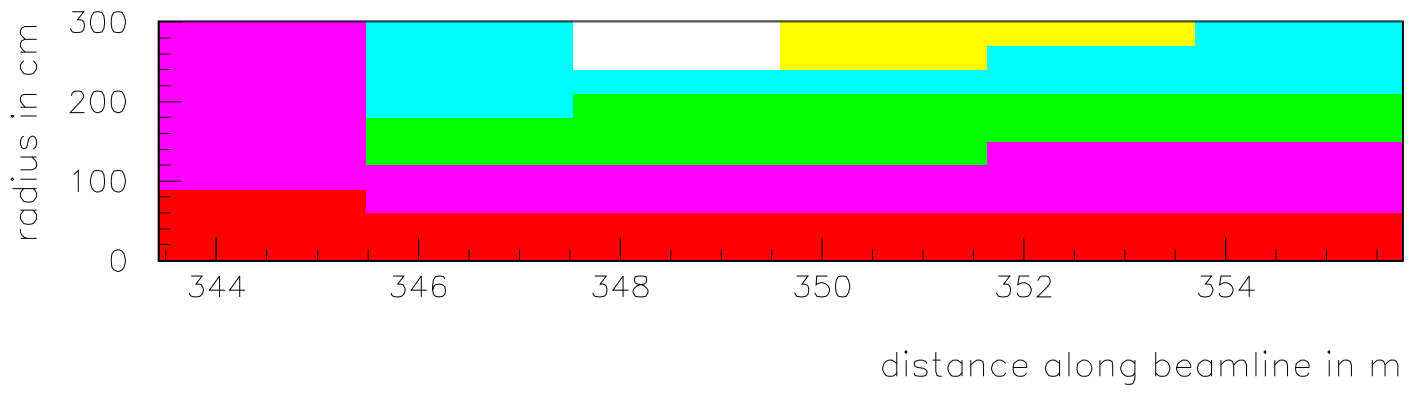

Star density. Wire scanners.

Figure 4.13: Star density distribution for last four regions. The top plot begins at the Pre-target gate. 

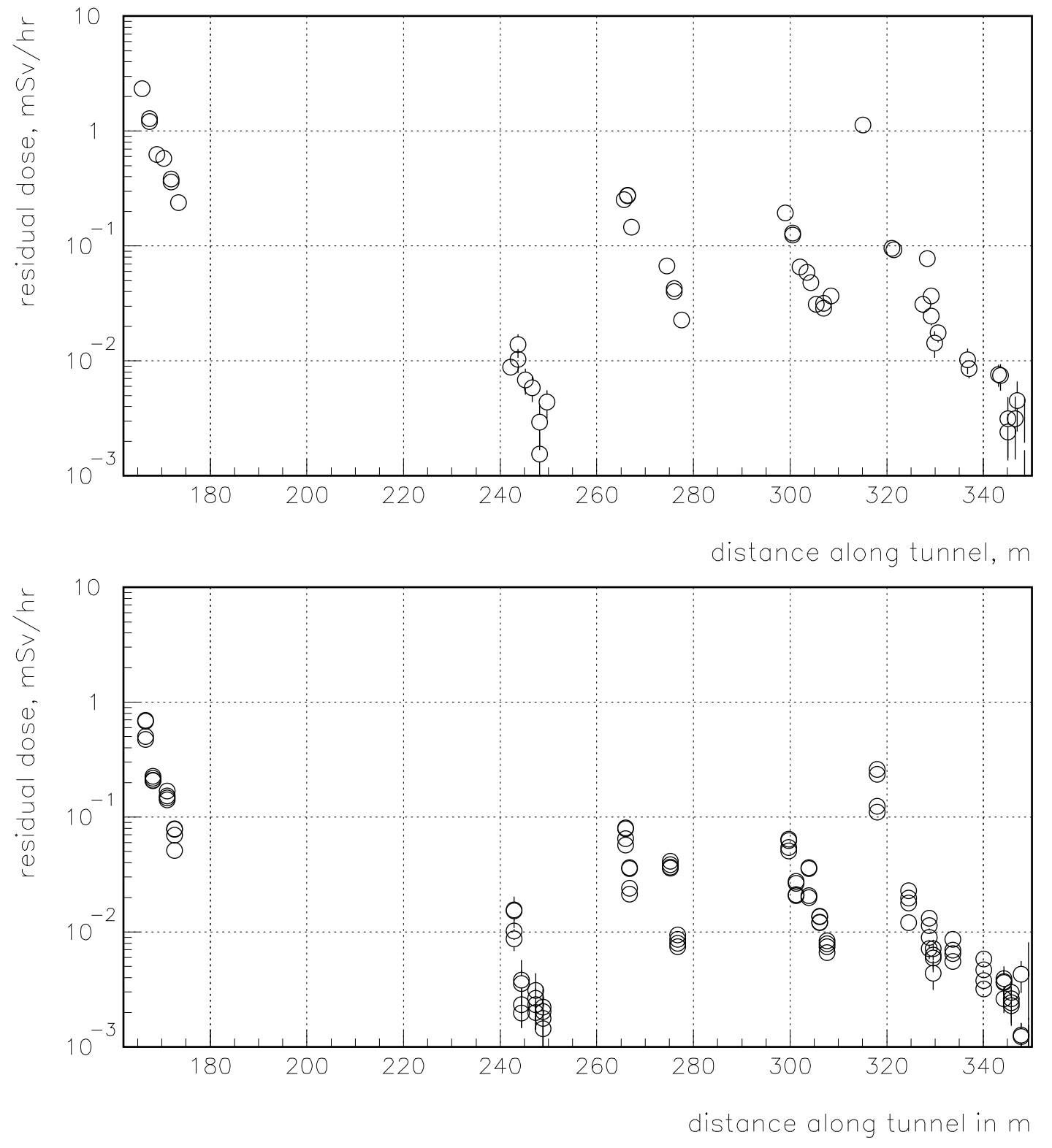

30 day of irradiation/1 day cooling residual dose at contact.

Figure 4.14: Residual dose from interaction of beam protons with all wire scanners IN ( $1 \mathrm{mSv} / \mathrm{hr}$ is equal to $100 \mathrm{mRem} / \mathrm{hr}$ ). (top) dose averaged over the front face of each magnet and (bottom) dose averaged along the side of each magnet 
Table.4.10 : Flux and prompt dose at the suface of magnets along the beamline from interactions with the wire scanners. The distance is measured from the upstream face of the first Lambertson magnet (see Fig. 2.1).

\begin{tabular}{|c|c|c|c|c|}
\hline $\begin{array}{l}\mathrm{Z} \\
(\mathrm{m})\end{array}$ & $\begin{array}{l}\text { neutrons } \\
\left(\mathrm{cm}^{-2} \mathrm{p}^{-1}\right)\end{array}$ & $\begin{array}{l}\text { charged hadrons } \\
\qquad\left(\mathrm{cm}^{-2} \mathrm{p}^{-1}\right)\end{array}$ & $\begin{array}{c}\text { Dose } \\
\left(\mathrm{mSv} \mathrm{p}^{-1}\right)\end{array}$ & $\begin{array}{l}\text { Dose Error } \\
\left(\mathrm{mSv} \mathrm{p}^{-1}\right)\end{array}$ \\
\hline 166.638 & $4.02220 \mathrm{E}-07$ & $1.03390 \mathrm{E}-07$ & $2.01025 \mathrm{E}-13$ & $3.99820 \mathrm{E}-15$ \\
\hline 168.162 & $1.56740 \mathrm{E}-07$ & 2.59310E-08 & $5.89600 \mathrm{E}-14$ & $2.01873 \mathrm{E}-15$ \\
\hline 171.111 & $1.07030 \mathrm{E}-07$ & 2.64700E-08 & $5.22475 \mathrm{E}-14$ & $2.70801 \mathrm{E}-15$ \\
\hline 172.635 & 4.97600E-08 & 8.62200E-09 & $1.95575 \mathrm{E}-14$ & $1.29979 \mathrm{E}-15$ \\
\hline 242.892 & 8.10800E-09 & 4.78690E-09 & $7.21350 \mathrm{E}-15$ & $1.22445 \mathrm{E}-15$ \\
\hline 244.416 & $1.81140 \mathrm{E}-09$ & $3.53420 \mathrm{E}-10$ & $5.36850 \mathrm{E}-16$ & $7.74645 \mathrm{E}-17$ \\
\hline 247.370 & $1.42680 \mathrm{E}-09$ & 7.10300E-10 & $7.97800 \mathrm{E}-16$ & $1.17803 \mathrm{E}-16$ \\
\hline 248.894 & $9.93300 \mathrm{E}-10$ & $8.18560 \mathrm{E}-10$ & $1.07730 \mathrm{E}-15$ & $3.26427 \mathrm{E}-16$ \\
\hline 265.981 & 4.28190E-08 & $1.95300 \mathrm{E}-08$ & $3.23100 \mathrm{E}-14$ & $7.56394 \mathrm{E}-16$ \\
\hline 266.743 & $2.08480 \mathrm{E}-08$ & 5.24180E-09 & $1.03170 \mathrm{E}-14$ & $3.45683 \mathrm{E}-16$ \\
\hline 275.213 & $2.40640 \mathrm{E}-08$ & 8.68600E-09 & $1.50800 \mathrm{E}-14$ & 4.30139E-16 \\
\hline 276.737 & $5.79600 \mathrm{E}-09$ & $1.32020 \mathrm{E}-09$ & $3.12625 \mathrm{E}-15$ & $2.75150 \mathrm{E}-16$ \\
\hline 299.718 & $3.95730 \mathrm{E}-08$ & $1.16980 \mathrm{E}-08$ & $2.06900 \mathrm{E}-14$ & $3.43889 \mathrm{E}-16$ \\
\hline 301.242 & $1.65880 \mathrm{E}-08$ & $3.11710 \mathrm{E}-09$ & $6.88575 \mathrm{E}-15$ & $2.30324 \mathrm{E}-16$ \\
\hline 303.869 & 1.89040E-08 & 5.99880E-09 & $1.05438 \mathrm{E}-14$ & $3.38311 \mathrm{E}-16$ \\
\hline 306.151 & $9.26300 \mathrm{E}-09$ & $2.42640 \mathrm{E}-09$ & $4.47475 \mathrm{E}-15$ & $2.42883 \mathrm{E}-16$ \\
\hline 307.675 & 5.34000E-09 & $9.44600 \mathrm{E}-10$ & $2.07425 \mathrm{E}-15$ & $1.30185 \mathrm{E}-16$ \\
\hline 318.047 & 1.18610E-07 & 4.40360E-08 & 7.79100E-14 & $1.58536 \mathrm{E}-15$ \\
\hline 324.472 & $1.25380 \mathrm{E}-08$ & 3.18020E-09 & $6.83725 \mathrm{E}-15$ & $3.60763 \mathrm{E}-16$ \\
\hline 328.793 & $6.75900 \mathrm{E}-09$ & 2.25410E-09 & $3.48225 \mathrm{E}-15$ & $3.35601 \mathrm{E}-16$ \\
\hline 329.555 & 3.47610E-09 & $9.90800 \mathrm{E}-10$ & $2.88100 \mathrm{E}-15$ & 4.43202E-16 \\
\hline 333.655 & 3.99770E-09 & 1.86170E-09 & $3.32475 \mathrm{E}-15$ & $2.02556 \mathrm{E}-16$ \\
\hline 340.081 & $2.57910 \mathrm{E}-09$ & $1.28170 \mathrm{E}-09$ & $2.43050 \mathrm{E}-15$ & $1.75595 \mathrm{E}-16$ \\
\hline 344.272 & $2.26090 \mathrm{E}-09$ & $8.17200 \mathrm{E}-10$ & $1.36680 \mathrm{E}-15$ & $1.49879 \mathrm{E}-16$ \\
\hline 345.796 & 1.66390E-09 & $8.46400 \mathrm{E}-10$ & $1.24925 \mathrm{E}-15$ & $1.74580 \mathrm{E}-16$ \\
\hline 347.749 & 1.12540E-09 & 6.37840E-10 & 8.93950E-16 & $1.04203 \mathrm{E}-16$ \\
\hline 349.273 & 1.13290E-09 & $1.01223 \mathrm{E}-09$ & $1.89555 \mathrm{E}-15$ & $3.64679 \mathrm{E}-16$ \\
\hline
\end{tabular}


Table.4.11 : Losses for each wire scanner (number per primary proton).

\begin{tabular}{|c|c|c|}
\hline Wire Scanner & $\begin{array}{c}\text { Loss } \\
\left(\mathrm{p}^{-1}\right)\end{array}$ & $\begin{array}{c}\text { Dist from L61 } \\
(\mathrm{m})\end{array}$ \\
\hline PM111 (wire) & $8.30 \mathrm{E}-5$ & 164.74 \\
PM112 (wire) & $7.23 \mathrm{E}-6$ & 173.85 \\
PM114 (wire) & $7.23 \mathrm{E}-6$ & 250.11 \\
PM115 (foil) & $7.23 \mathrm{E}-6$ & 265.01 \\
PM117 (foil) & $7.23 \mathrm{E}-6$ & 298.33 \\
PM118 (foil) & $8.30 \mathrm{E}-5$ & 309.11 \\
PM121 (foil) & $7.23 \mathrm{E}-6$ & 351.86 \\
PMTGT (foil) & $7.23 \mathrm{E}-6$ & 364.31 \\
\hline
\end{tabular}




\subsection{Beam Loss Correlations}

There are no magnetic elements in first two regions. The main source of radiation there is from beam loss near the HQ111 or HQ112 quadrupoles. It is natural to suppose the following simple relations:

$$
\begin{aligned}
& S_{1}=\alpha_{1}^{111} \cdot L_{H Q 111} \\
& S_{1}=\alpha_{1}^{112} \cdot L_{H Q 112} \\
& S_{2}=\alpha_{2}^{111} \cdot L_{H Q 111} \\
& S_{2}=\alpha_{2}^{112} \cdot L_{H Q 112}
\end{aligned}
$$

where $S_{1}, S_{2}$ are average star densities in regions 1,2 and $L_{H Q 111}, L_{H Q 112}$ are number of protons lost on HQ111, HQ112, respectively. For other regions we suppose

$$
S_{i}=\alpha_{i} \cdot L_{i}
$$

where $S_{i}$ is average star density in region $i$ and $L_{i}$ is beam loss in this region. Using data from tables 4.3 and 4.5 one can estimate the correlation constants. Results are presented in tables 4.12-13. It is seen that in most cases $\alpha_{i}$ has a weak dependence on the loss scenario.

Using the above relations, constants from tables 4.12-13 and star density limits, it is possible to estimate the maximum beam loss which could be allowed on different magnets. Acceptable beam loss limits are presented in table 4.14. 
Table.4.12 : Constants for estimation of the average star density (star/cc/proton) in $99.9 \%$ volume.

\begin{tabular}{lllll}
\hline source & $\alpha_{1}^{111}$ & $\alpha_{1}^{112}$ & $\alpha_{2}^{111}$ & $\alpha_{2}^{112}$ \\
\hline HV101 1.20\% & & $2.3 \times 10^{-8}$ & & $7.3 \times 10^{-9}$ \\
\hline HV101 1.25\% & & $2.1 \times 10^{-8}$ & $6.5 \times 10^{-9}$ \\
\hline HV101 1.40\% & & $1.4 \times 10^{-8}$ & $4.0 \times 10^{-9}$ \\
\hline HV101 1.50\% & & $1.5 \times 10^{-8}$ & & $5.6 \times 10^{-9}$ \\
\hline V108 0.75\% & $9.3 \times 10^{-9}$ & & $1.9 \times 10^{-9}$ & \\
\hline V108 0.78\% & $1.0 \times 10^{-8}$ & & $1.8 \times 10^{-9}$ & \\
\hline V108 0.85\% & $1.6 \times 10^{-8}$ & & $3.6 \times 10^{-9}$ & \\
\hline V108 0.90\% & $1.7 \times 10^{-8}$ & & $6.5 \times 10^{-9}$ & \\
\hline comb 1 & & $1.6 \times 10^{-8}$ & & $4.9 \times 10^{-9}$ \\
\hline comb 2 & $1.1 \times 10^{-8}$ & & $4.3 \times 10^{-9}$ & \\
\hline comb 3 & $0.8 \times 10^{-8}$ & & $2.2 \times 10^{-9}$ & \\
\hline wire & $1.8 \times 10^{-8}$ & $1.8 \times 10^{-8}$ & $4.2 \times 10^{-9}$ & $4.2 \times 10^{-9}$ \\
\hline \hline average & $1.3 \times 10^{-8}$ & $1.8 \times 10^{-8}$ & $3.5 \times 10^{-9}$ & $5.4 \times 10^{-9}$ \\
\hline \hline
\end{tabular}

Table.4.13 : Constants for estimation of the average star density (star/cc/proton) in $99.9 \%$ volume.

\begin{tabular}{|c|c|c|c|c|c|}
\hline source & $\alpha_{3}$ & $\alpha_{4}$ & $\alpha_{5}$ & $\alpha_{6}$ & $\alpha_{7}$ \\
\hline HV101 1.20\% & & $8.1 \times 10^{-8}$ & $2.7 \times 10^{-8}$ & & \\
\hline HV101 $1.25 \%$ & & $7.6 \times 10^{-8}$ & $2.8 \times 10^{-8}$ & & \\
\hline HV101 1.40\% & & $8.9 \times 10^{-8}$ & & & \\
\hline HV101 1.50\% & & & & & \\
\hline V108 $0.75 \%$ & & & & $2.3 \times 10^{-8}$ & $1.5 \times 10^{-8}$ \\
\hline V108 0.78\% & & & & $1.3 \times 10^{-8}$ & $1.6 \times 10^{-8}$ \\
\hline V108 0.85\% & & & & $1.8 \times 10^{-8}$ & \\
\hline V108 0.90\% & & & & & \\
\hline comb 1 & & & & & \\
\hline comb 2 & $1.3 \times 10^{-7}$ & $9.9 \times 10^{-8}$ & & & \\
\hline comb 3 & & & & $2.0 \times 10^{-8}$ & \\
\hline wire & $1.5 \times 10^{-7}$ & $7.7 \times 10^{-8}$ & & & $2.2 \times 10^{-8}$ \\
\hline average & $1.4 \times 10^{-7}$ & $8.4 \times 10^{-8}$ & $2.8 \times 10^{-8}$ & $1.9 \times 10^{-8}$ & $1.8 \times 10^{-8}$ \\
\hline
\end{tabular}


Table.4.14 : Maximum acceptable losses from operational beam loss limit.

\begin{tabular}{llll}
\hline region & $\begin{array}{l}\text { star density limit } \\
\text { star/cc/proton }\end{array}$ & $\begin{array}{l}\text { beam loss limit } \\
\text { particle/proton }\end{array}$ & place \\
\hline 1 & $6.4 \times 10^{-4}$ & 1 & HQ111,HQ112 \\
\hline 2 & $2.1 \times 10^{-12}$ & $6.0 \times 10^{-4}$ & HQ111 \\
\hline 2 & $2.1 \times 10^{-12}$ & $4.0 \times 10^{-4}$ & HQ112 \\
\hline 3 & $5.7 \times 10^{-10}$ & $4.1 \times 10^{-3}$ & HQ113 \\
\hline 4 & $4.8 \times 10^{-10}$ & $5.7 \times 10^{-3}$ & V118-1 or HT117 \\
\hline 5 & $6.4 \times 10^{-10}$ & $2.3 \times 10^{-2}$ & V118-2 \\
\hline 6 & $6.4 \times 10^{-10}$ & $3.4 \times 10^{-2}$ & V118-4 \\
\hline 7 & $6.4 \times 10^{-10}$ & $3.6 \times 10^{-2}$ & HT121 \\
\hline
\end{tabular}




\section{Appendix A}

\section{MARS Model Elements}

This Appendix contains drawings of the MARS model beamline, showing transverse and longitudinal cross sections of magnets and cross sections of the NuMI tunnel as modeled. 

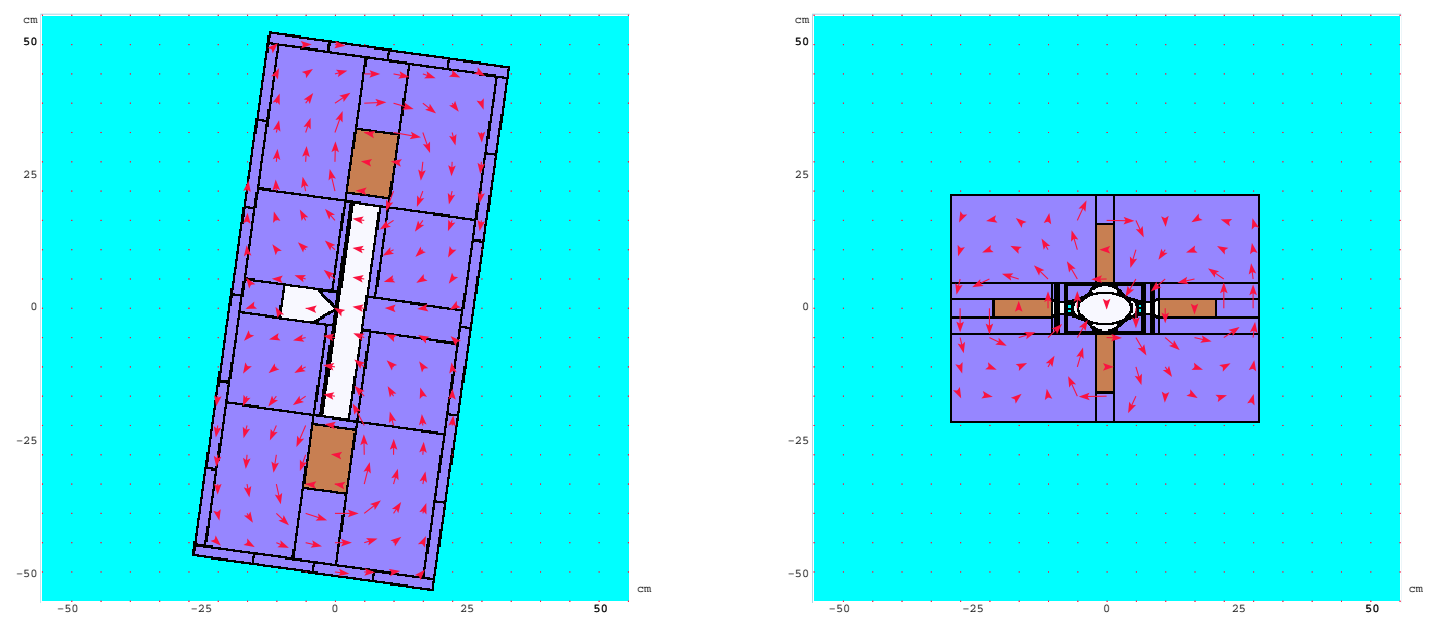

$\stackrel{x}{\llcorner} \times$

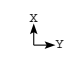

Figure A.1: LAM60 (Lambertson) and Q608 (3Q84) magnets. 


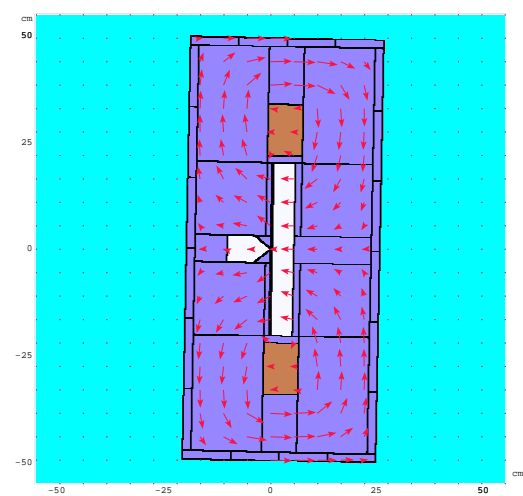

$x^{x}$

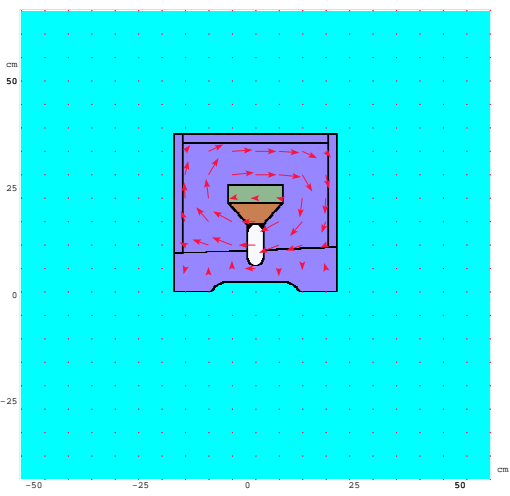

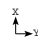

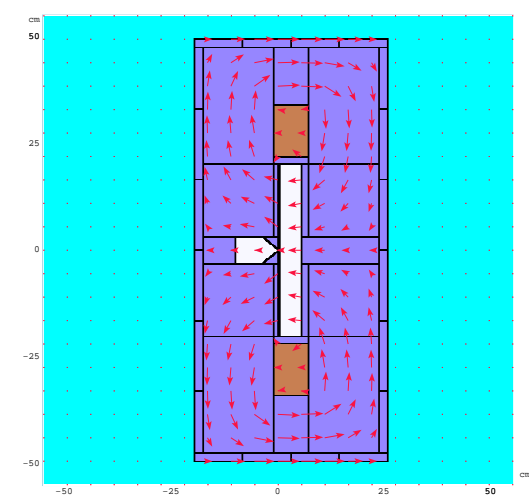

$\stackrel{x}{\llcorner} \times$

Figure A.2: LAM61A and LAM61B (Lambertson) magnets.

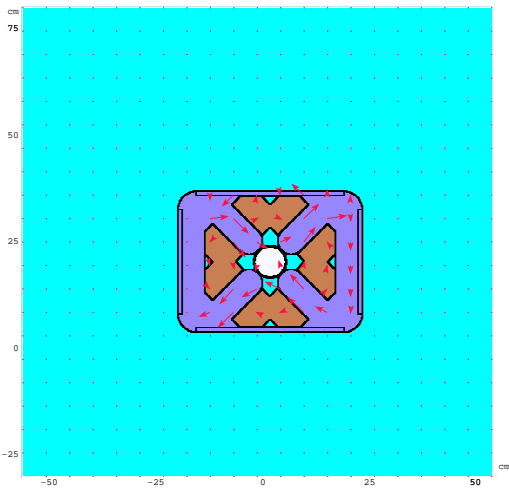

$L^{x}$

Figure A.3: V100 (C) and Q101 (3Q120-old) magnets. 


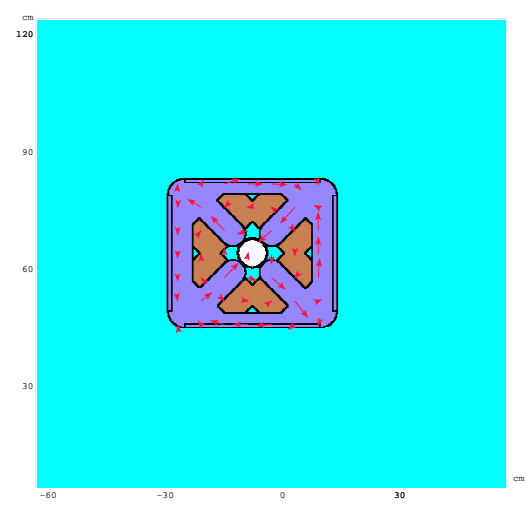

$\iota_{*}$

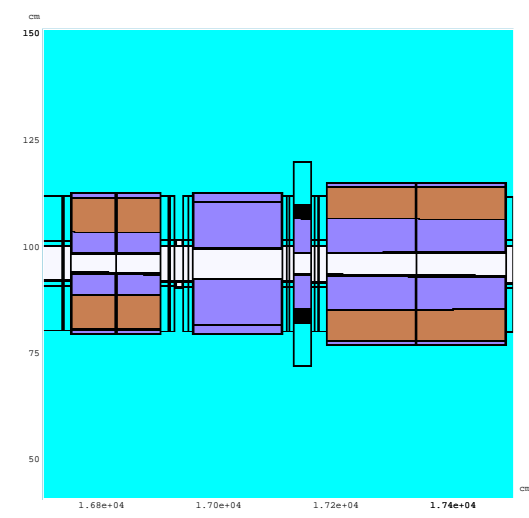

$L_{2}$

Figure A.4: Q102(3Q120) and Q104(3Q60)-H104(trim)-HT105(trim)-Q105(3Q120) magnets.

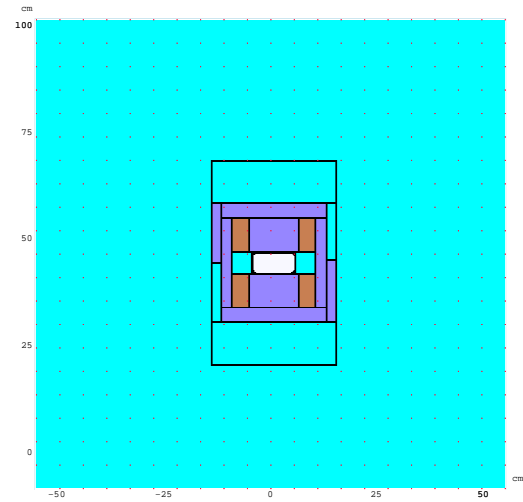

$\stackrel{x}{\longrightarrow}$

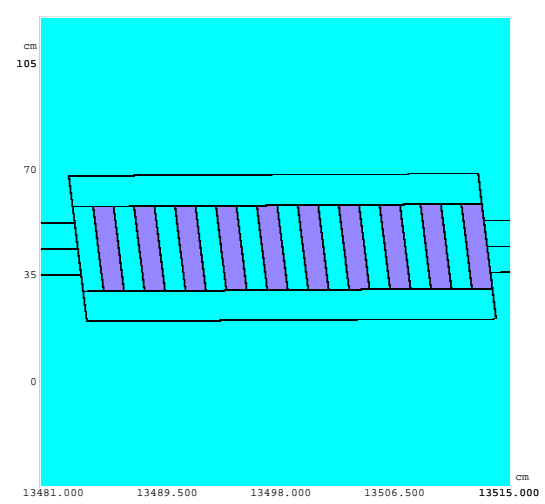

$\stackrel{x}{\llcorner}:$

Figure A.5: HT102 (trim) magnet. 

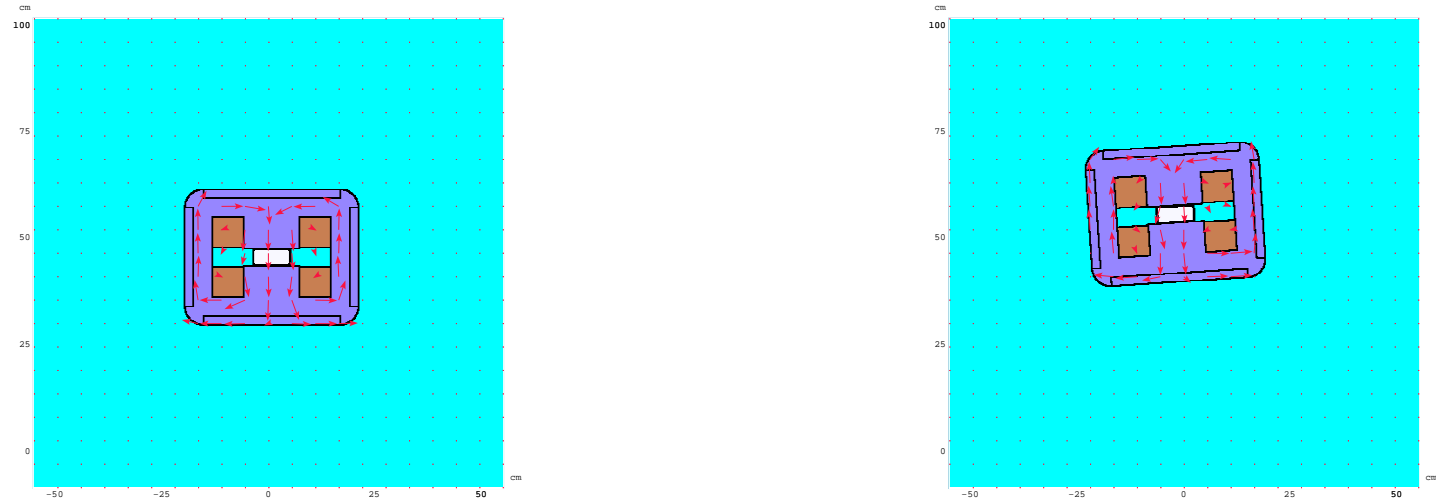

$\iota^{x}$ $\iota_{\llcorner}^{x}$

Figure A.6: HV101-1 and HV101-2 (EPB) magnets.
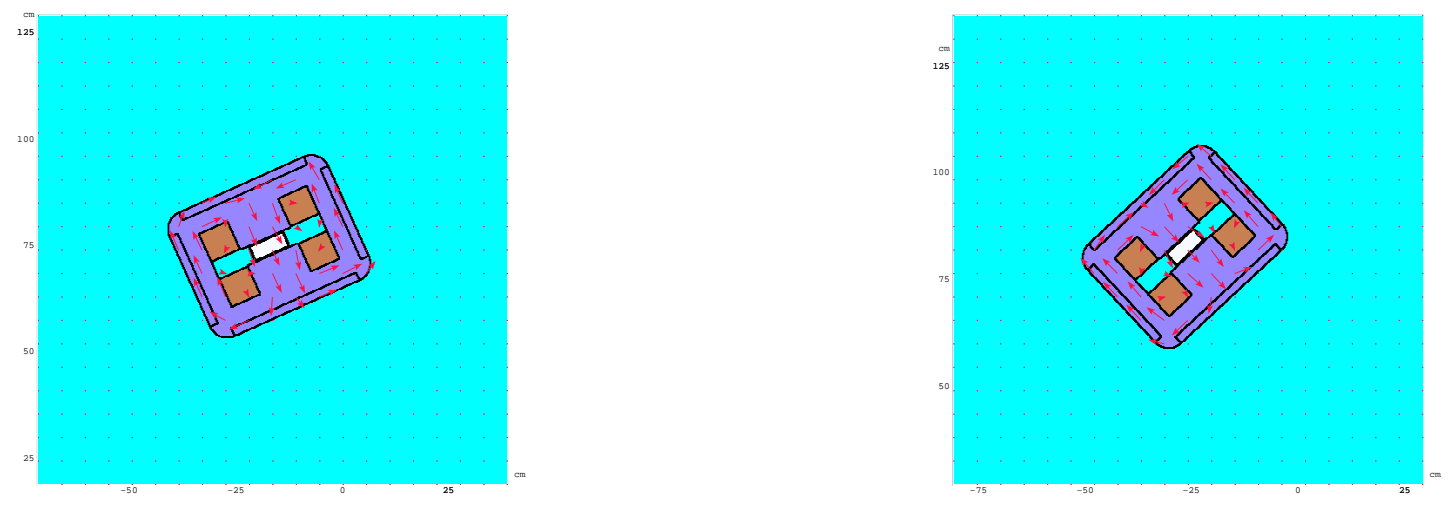

$\omega_{\infty}$ $L_{*}$

Figure A.7: HV101-3 and HV101-4 (EPB) magnets. 


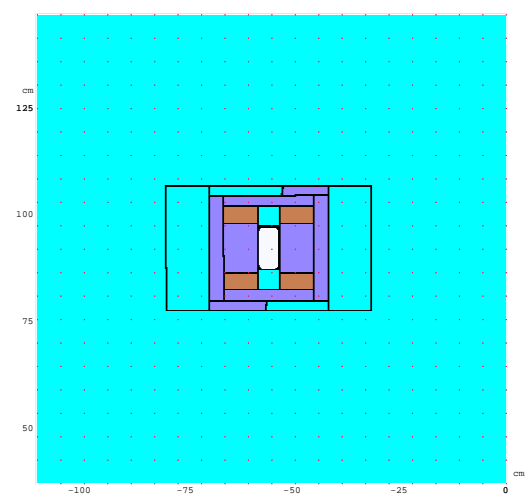

$\iota_{*}$

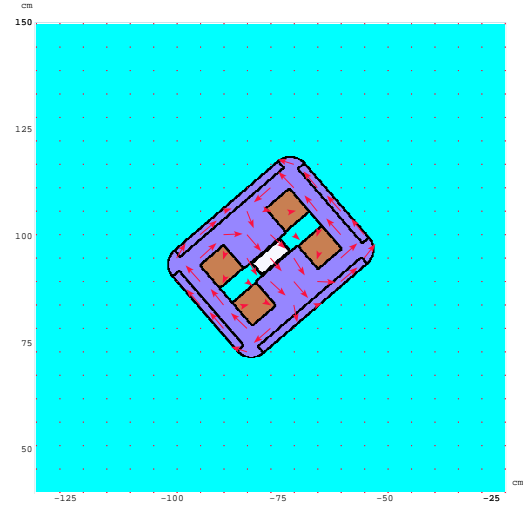

$\omega^{*}$

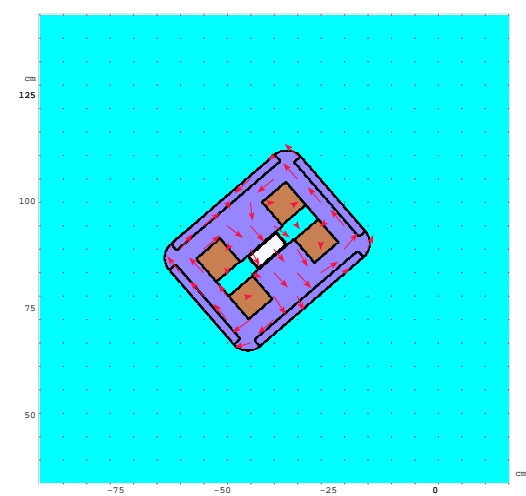

$L_{*}$

Figure A.8: VT103 (trim) and HV101-5 (EPB) magnets.

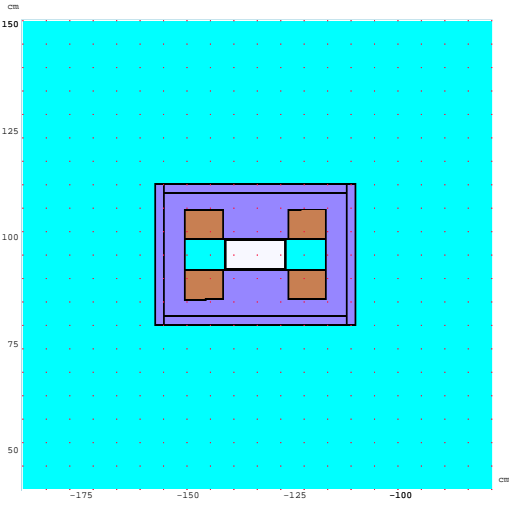

$x^{x}$

Figure A.9: HV101-6 (EPB) and H104 (trim dipole) magnets. 

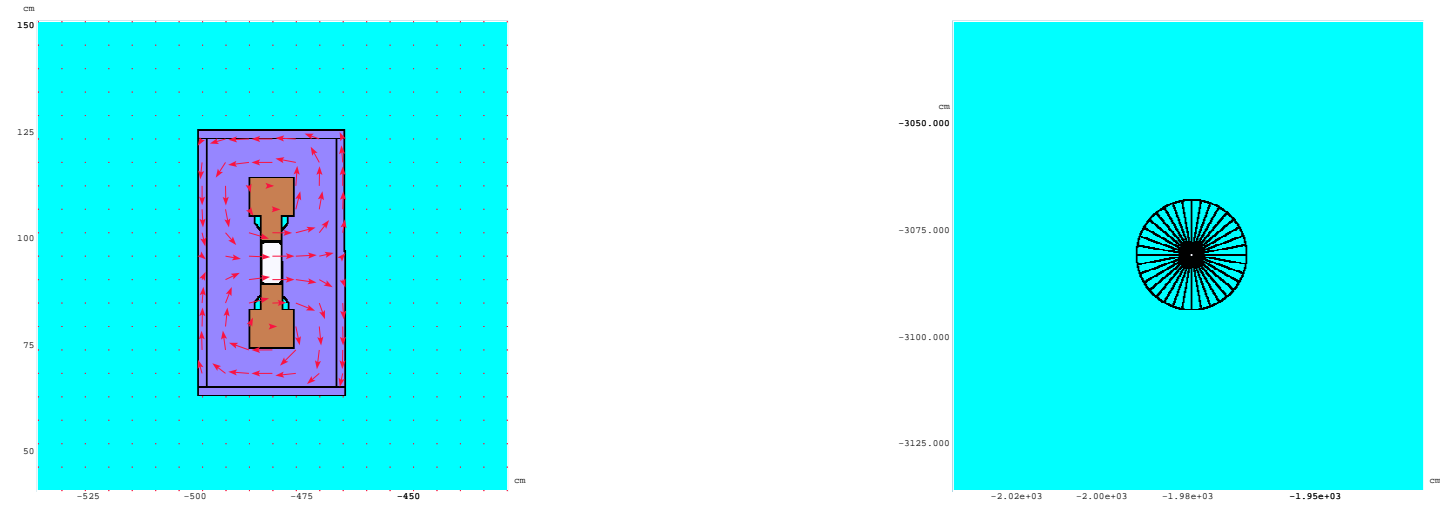

$\bigsqcup_{i}^{x}$

$\bigsqcup_{\llcorner}^{x}$

Figure A.10: V108-1 (B2) magnet and baffle. 


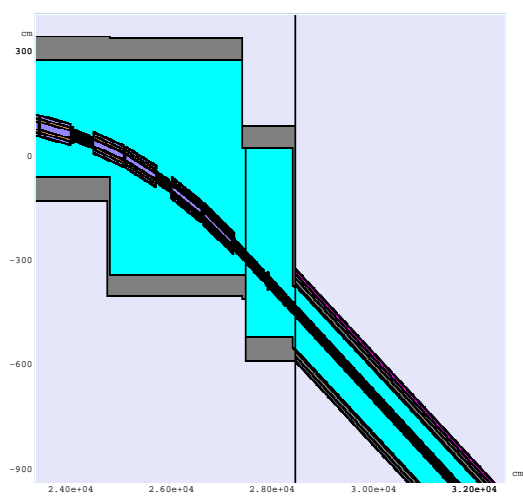

$\iota_{\rightarrow 2}^{x}$

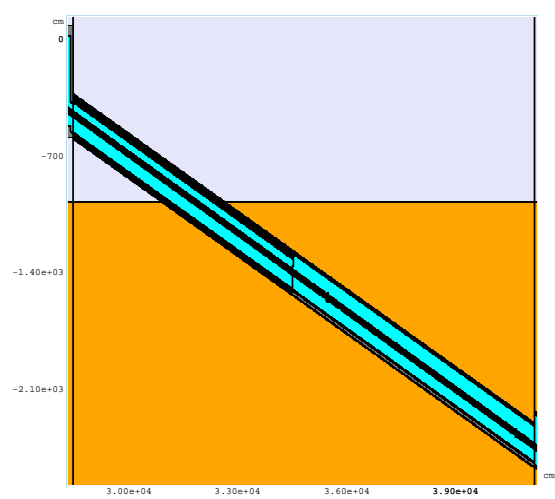

$\stackrel{x}{\hookrightarrow} 2$

Figure A.11: Extraction enclosure, extraction enclosure extension and carrier tunnel. 


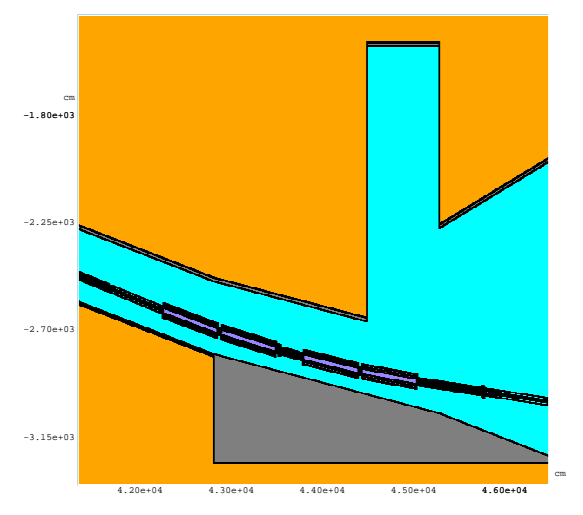

$\iota_{2}^{x}$

Figure A.12: Pretarget hall.
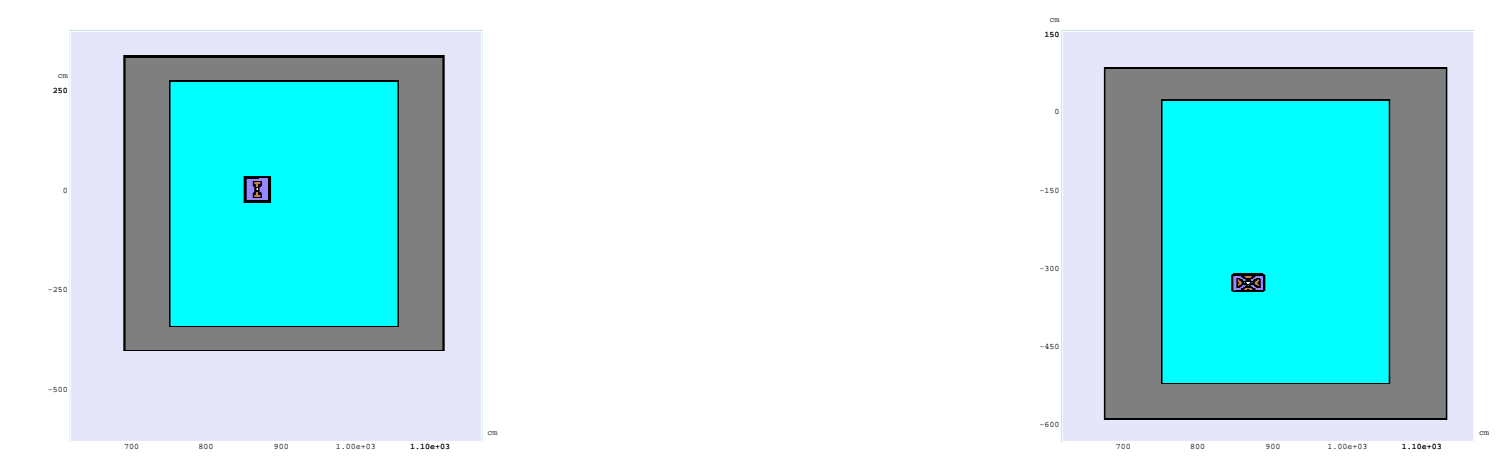

Ł.

L.

Figure A.13: Transverse cross section of NuMI tunnel in extraction enclosure (137 m) and extraction enclosure extension $(164 \mathrm{~m})$. 

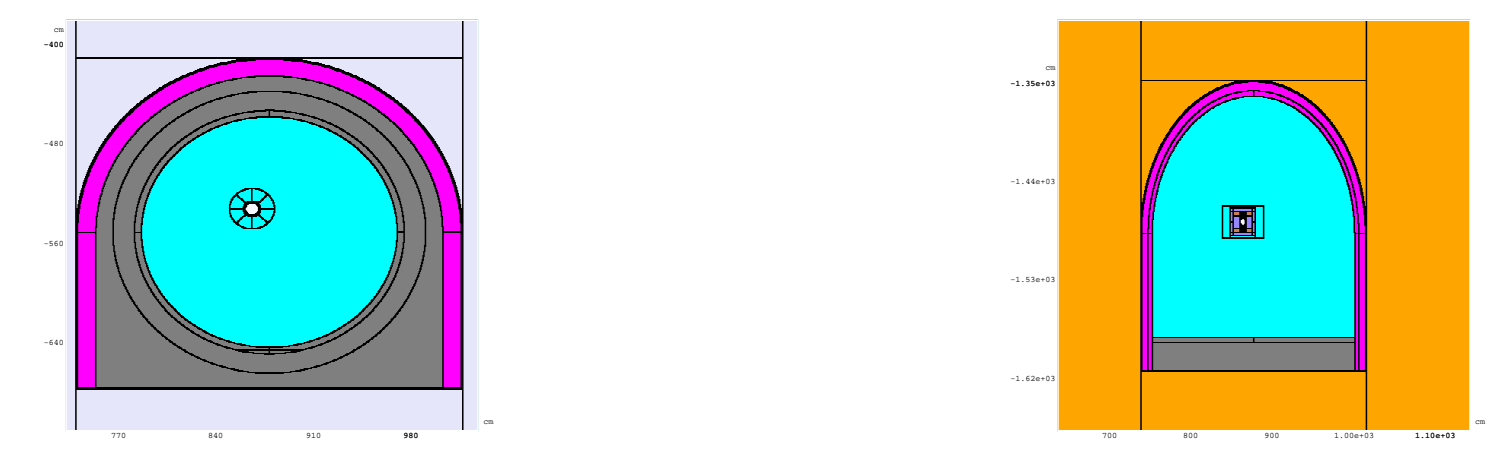

L.

L:

Figure A.14: Transverse cross section of NuMI tunnel in carrier tunnel $177 \mathrm{~m}$ and at $237 \mathrm{~m})$.

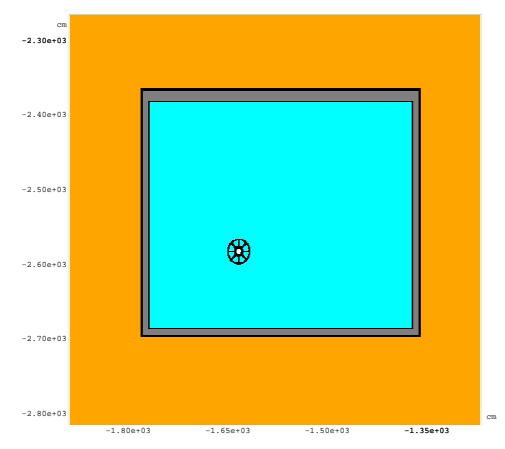

Ł.

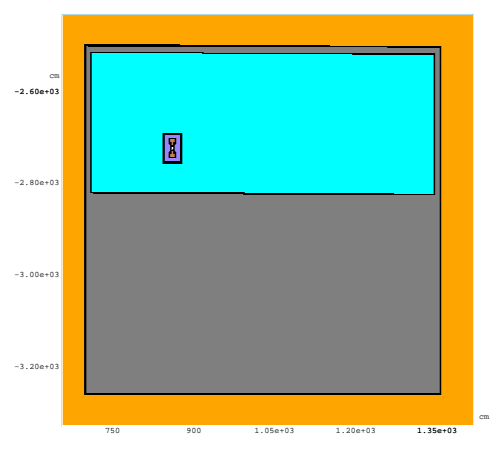

$L^{x}$

Figure A.15: Transverse cross section of NuMI tunnel in carrier tunnel $307 \mathrm{~m}$ and at $317 \mathrm{~m})$. 


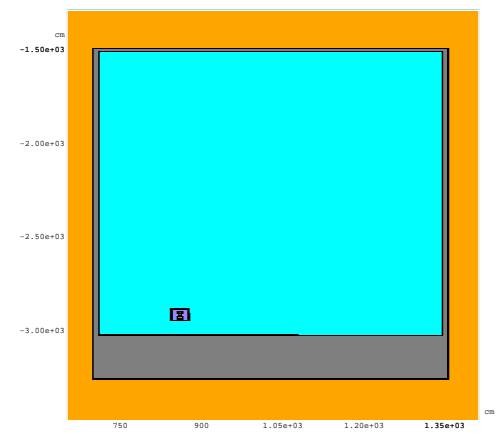

$\stackrel{x}{\leftrightarrow}$

Figure A.16: Transverse cross section of NuMI tunnel in carrier tunnel $337 \mathrm{~m}$ and at $347 \mathrm{~m})$.

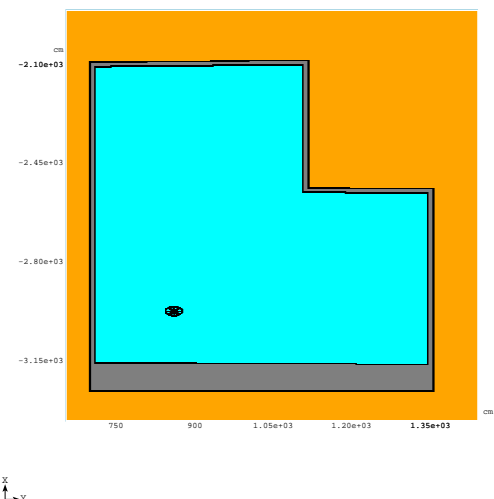

$\stackrel{x}{\hookrightarrow}$ 


\section{Appendix B}

\section{Star Density Distributions}

Star densities and residual dose distributions along the NuMI beamline for nine loss scenarios are presented this Appendix. For each case, four figures are shown: star density just within $(1 \mathrm{~cm})$ the tunnel wall for regions 1 through 3 , and 4 through 7 , spatial star density distribution for regions 1 through 3 , and 4 through 7 . 

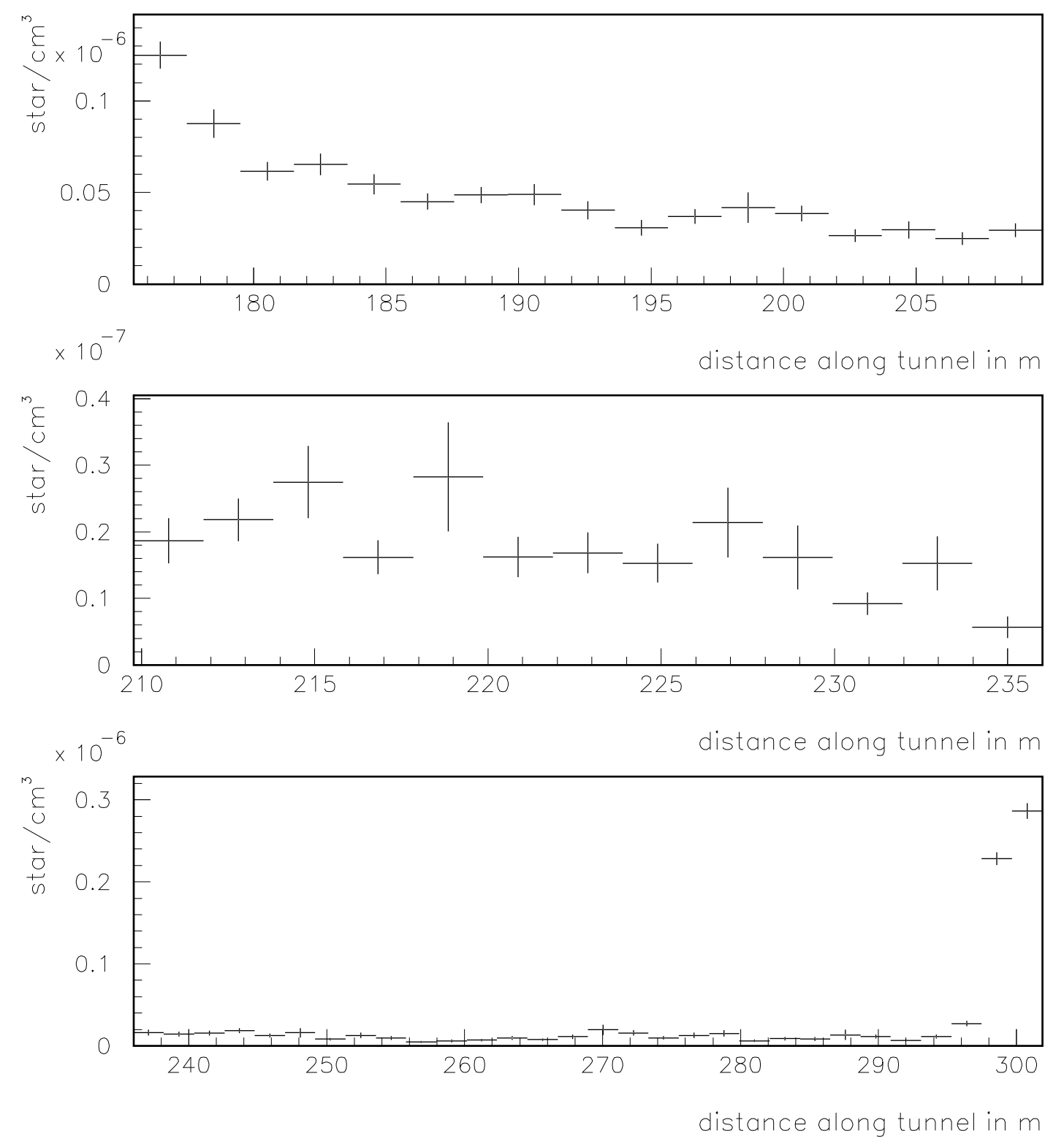

Star density distribution in $1 \mathrm{~cm}$ bin.

Figure B.1: Star density distribution for first three regions. Magnet current variation on $1.25 \%$ in HV101. 

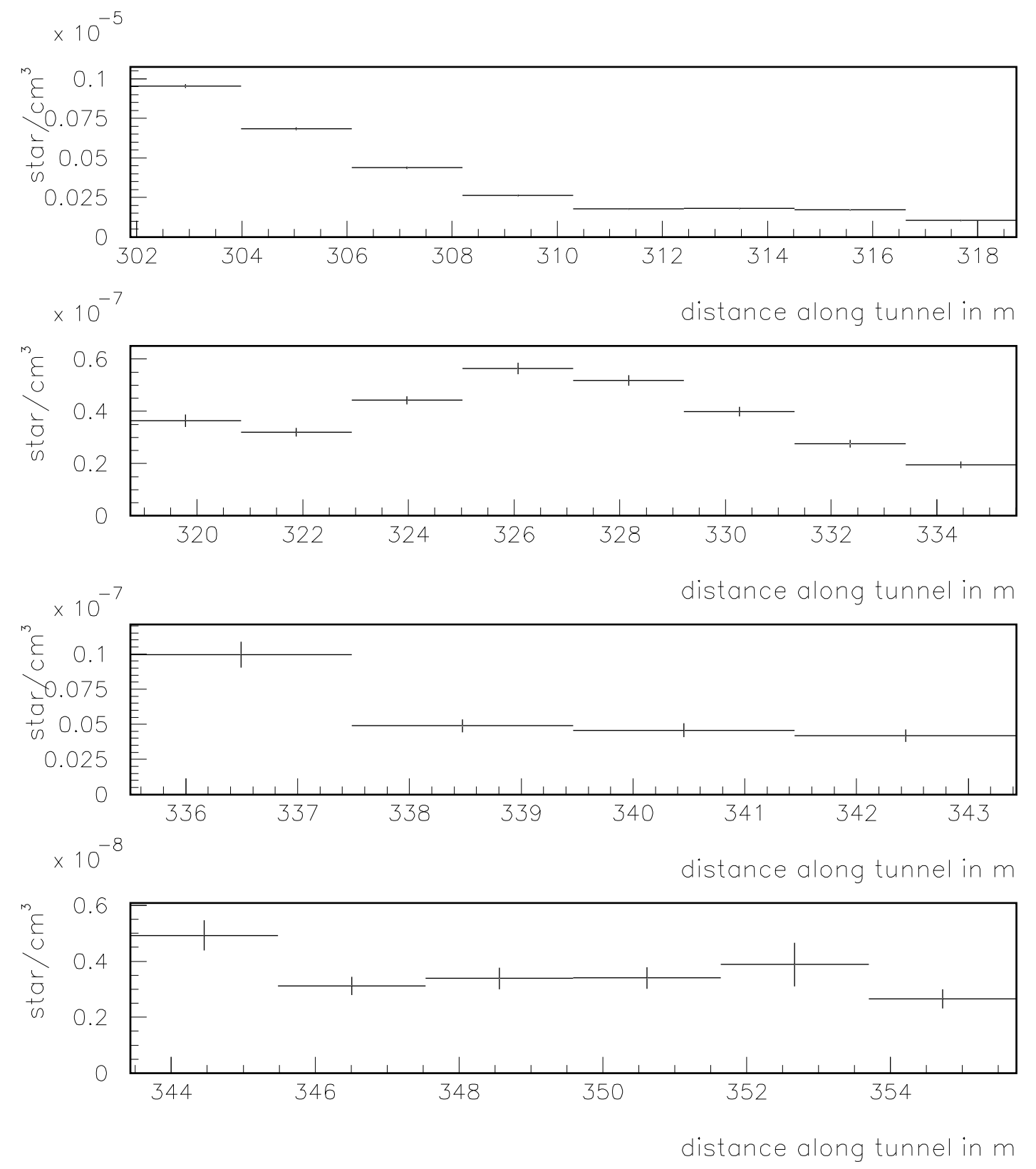

Star density distribution in $1 \mathrm{~cm}$ bin.

Figure B.2: Star density distribution for last four regions. Magnet current variation on $1.25 \%$ in HV101. 

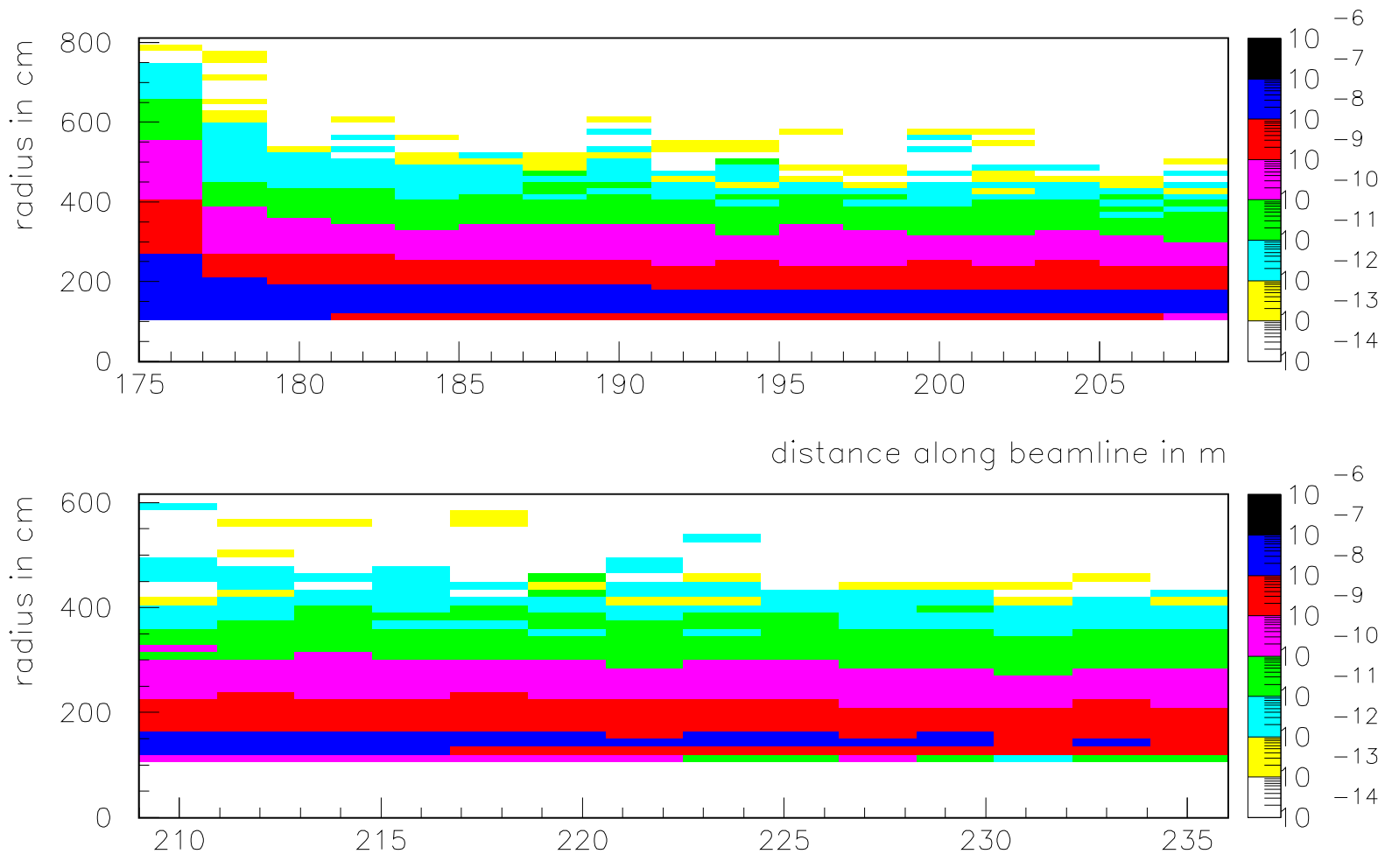

distance along beamline in $m$

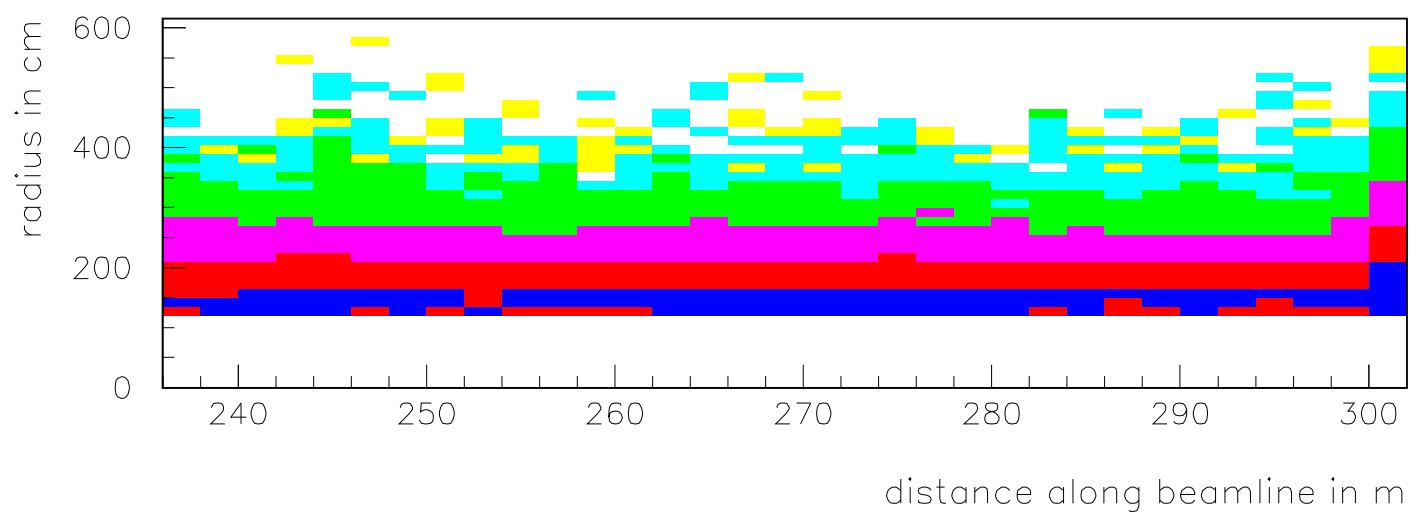

Star density distribution. $1.25 \%$ magnetic field variation in HV101

Figure B.3: Star density distribution for first three regions. 

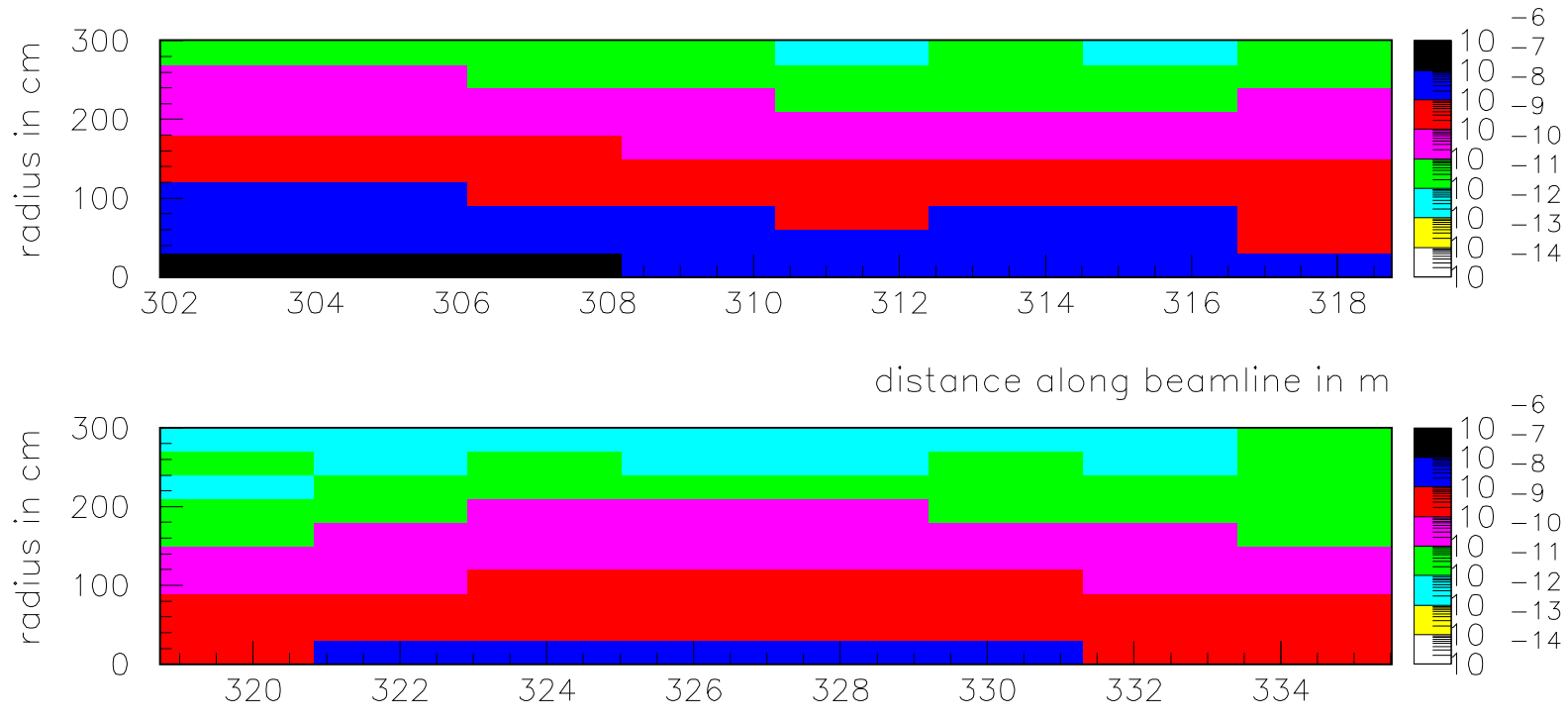

distance along beamline in $\mathrm{m}$

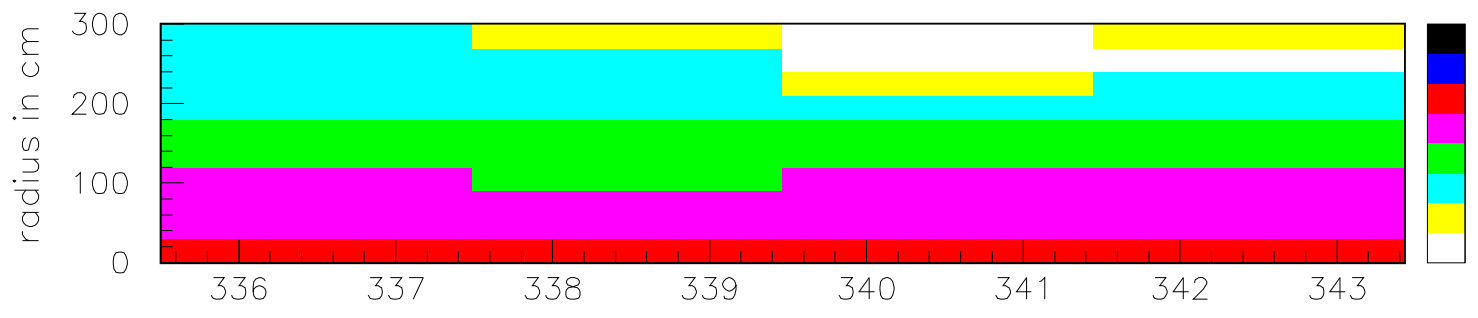

distance along beamline in $\mathrm{m}$

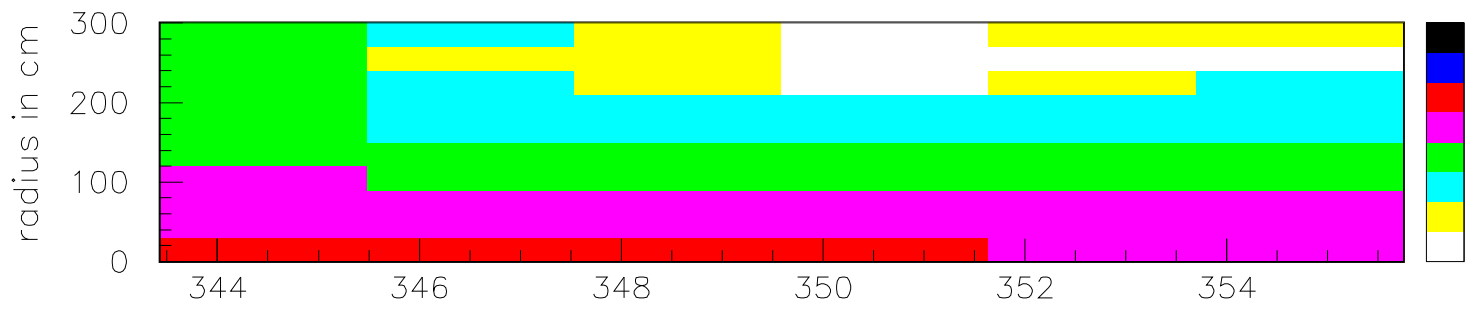

distance along beamline in $m$

Star density distribution. 1.25\% magnetic field variation in HV101

Figure B.4: Star density distribution for last four regions. 

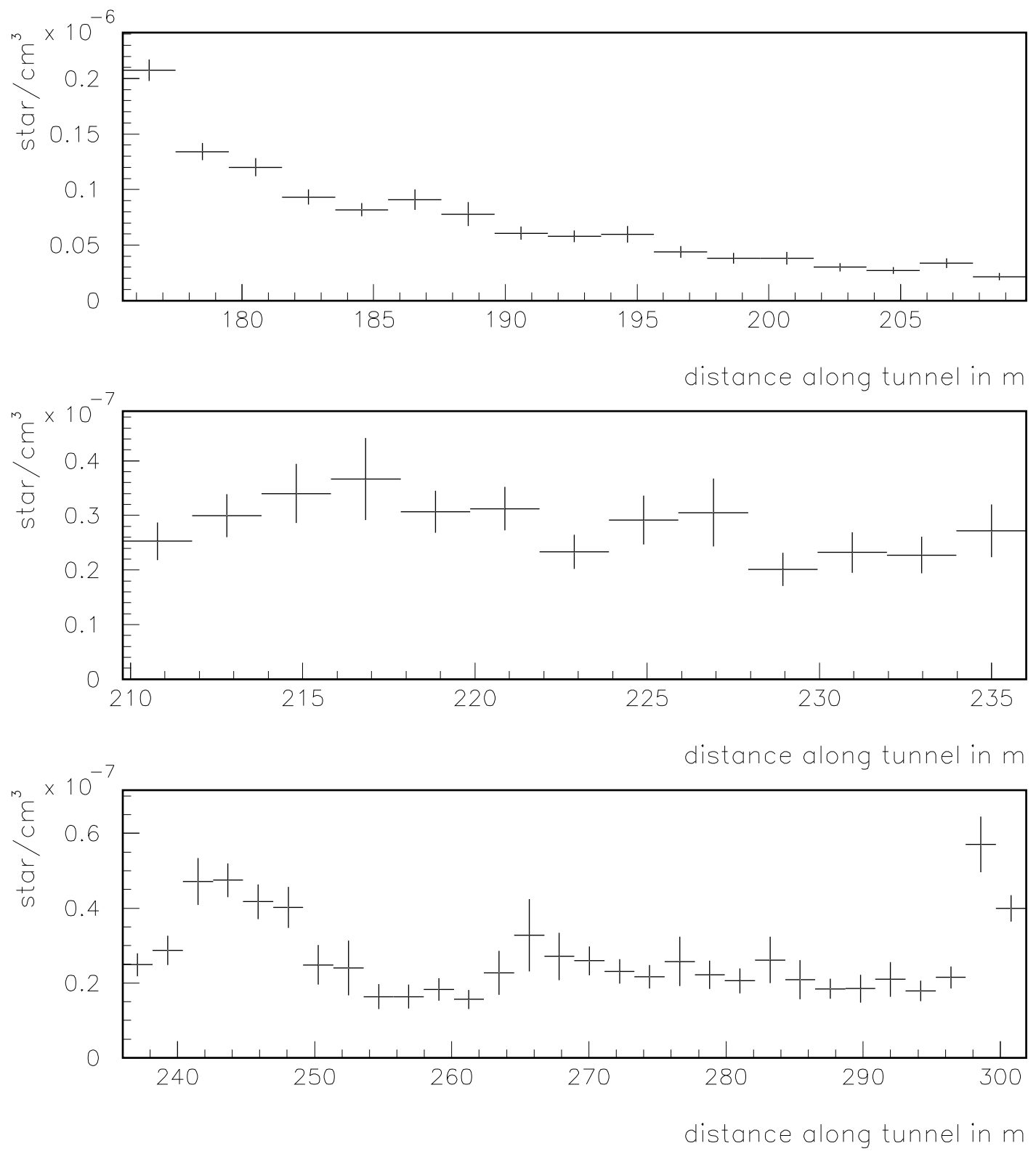

Star density distribution in $1 \mathrm{~cm}$ bin.

Figure B.5: Star density distribution for first three regions. Magnet current variation on $1.4 \%$ in HV101. 

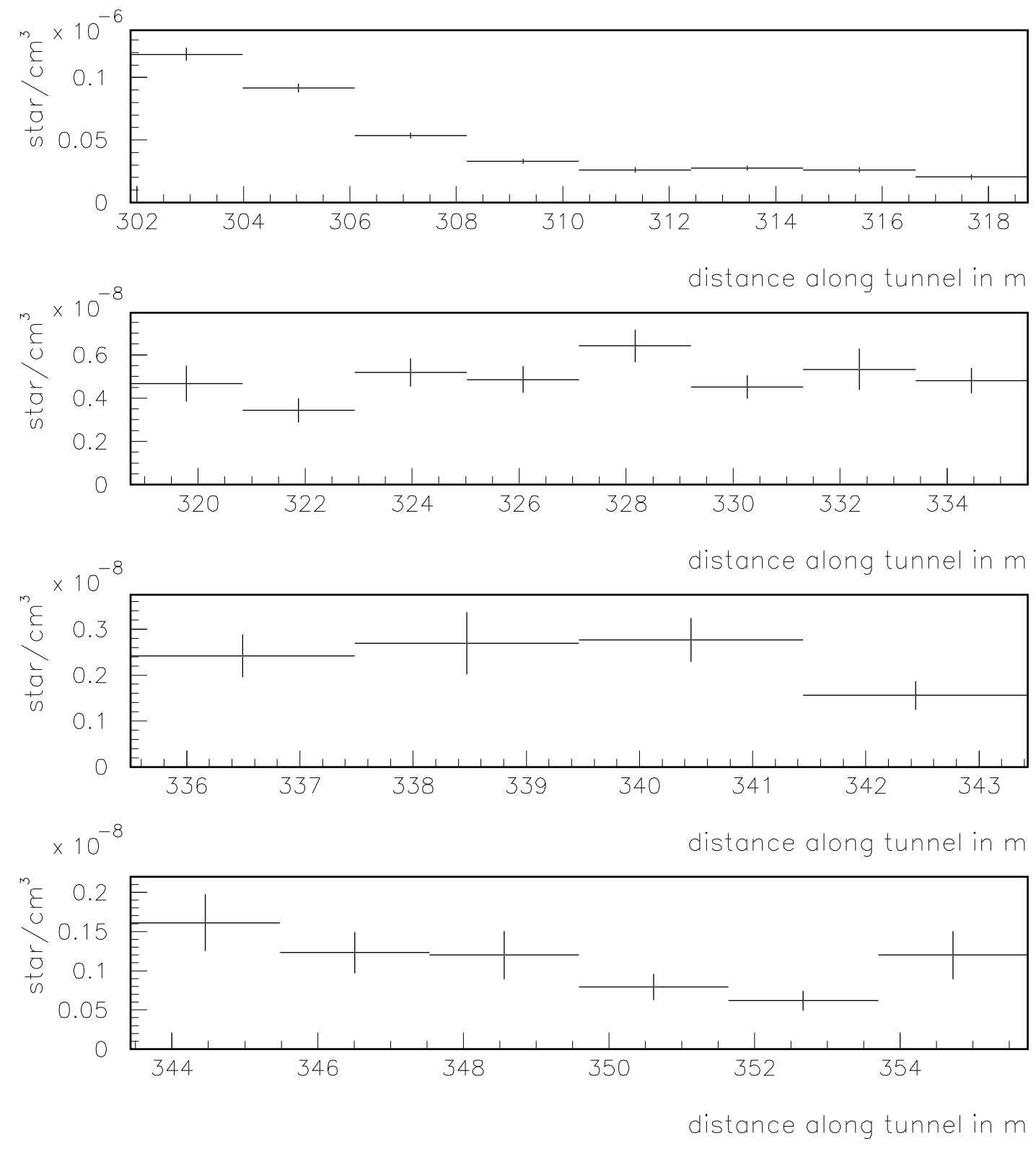

Star density distribution in $1 \mathrm{~cm}$ bin.

Figure B.6: Star density distribution for last four regions. Magnet current variation on $1.4 \%$ in HV101. 

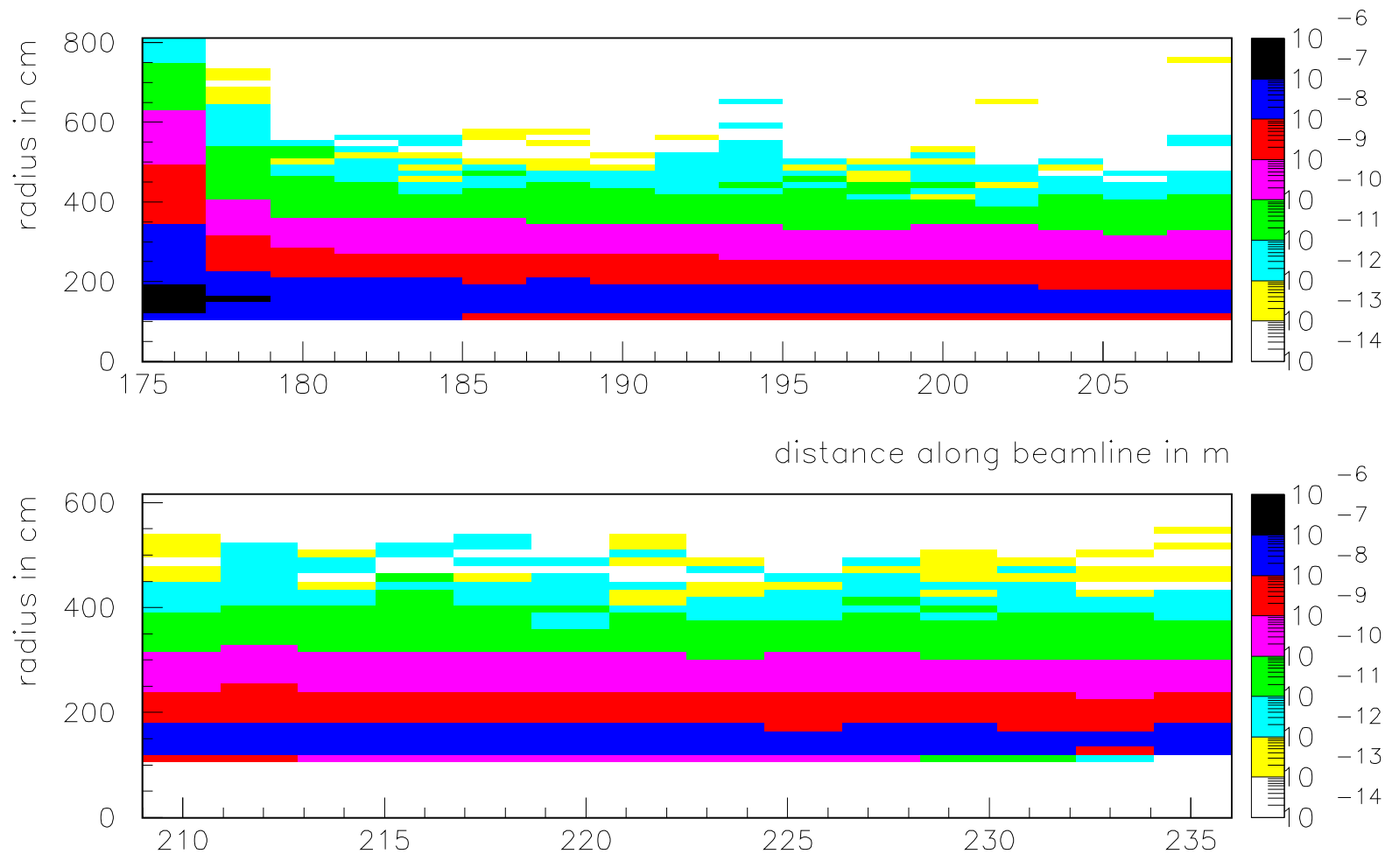

distance along beamline in $\mathrm{m}$

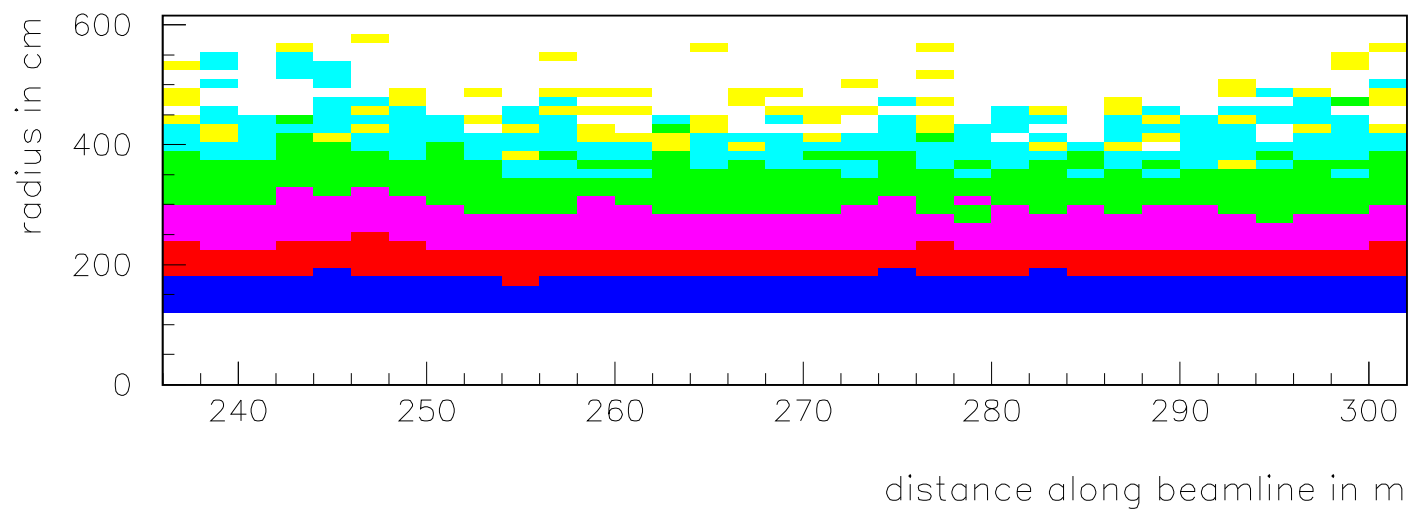

Star density distribution. 1.4\% magnetic field variation in HV101

Figure B.7: Star density distribution for first three regions. 

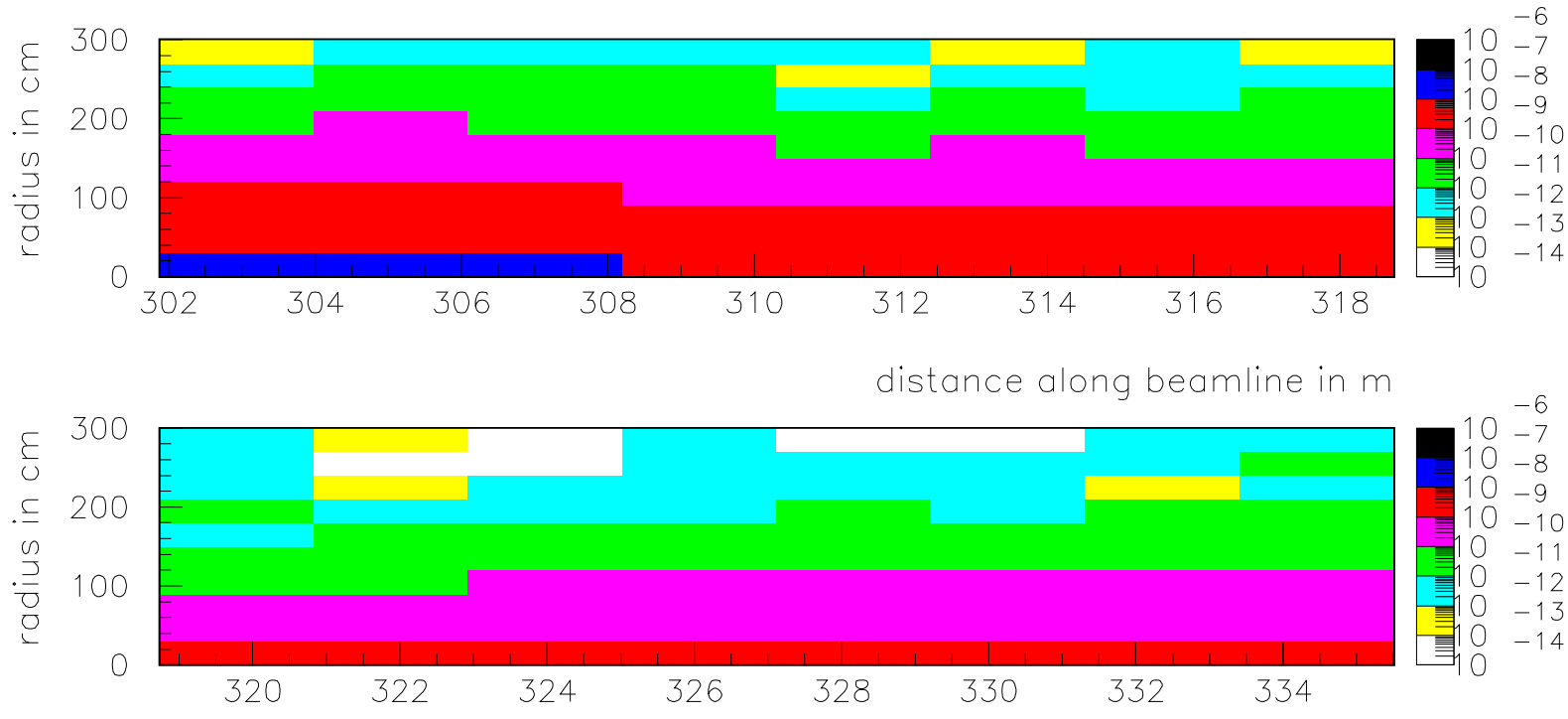

distance along beamline in $\mathrm{m}$

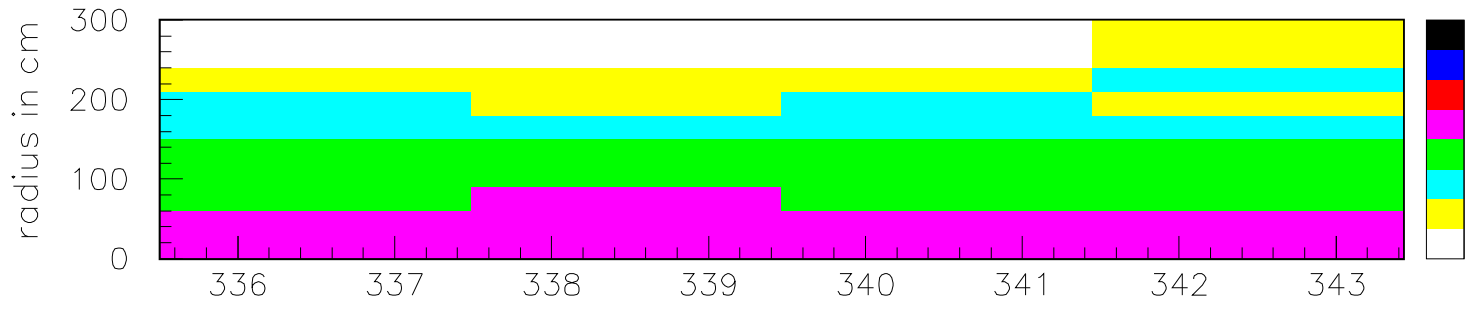

distance along beamline in $\mathrm{m}$

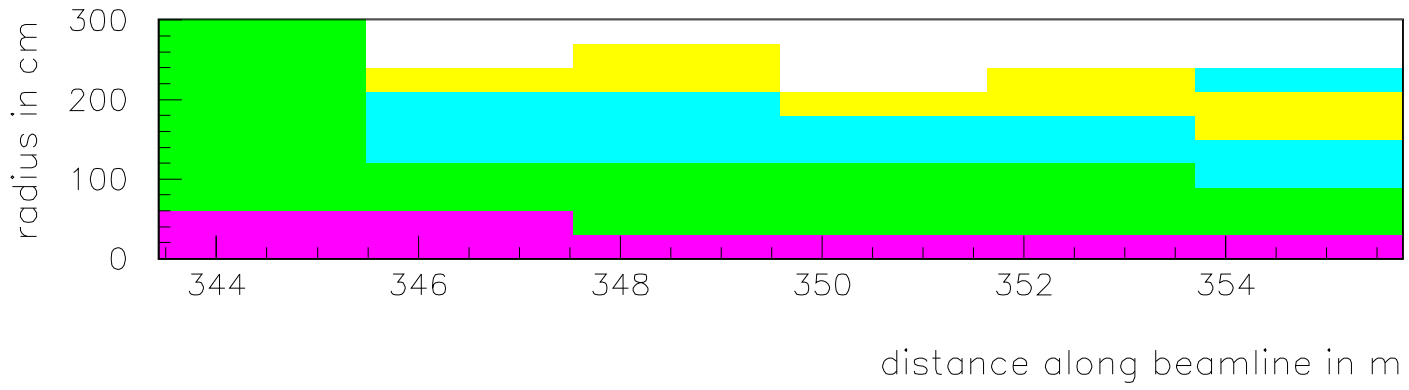

Star density distribution. 1.40\% magnetic field variation in HV101

Figure B.8: Star density distribution for last four regions. 


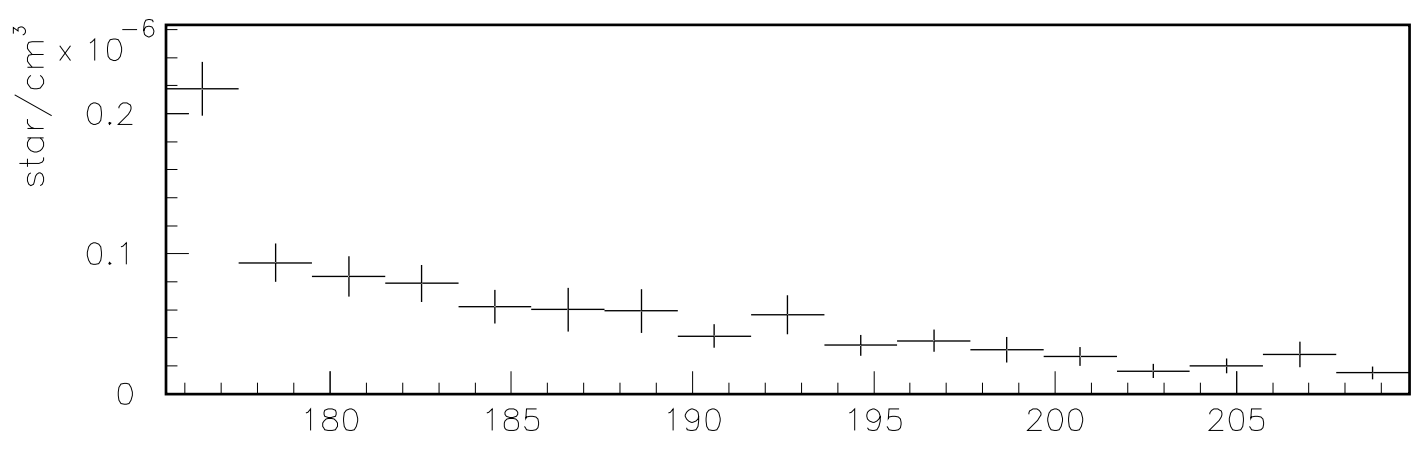

distance along tunnel in $m$

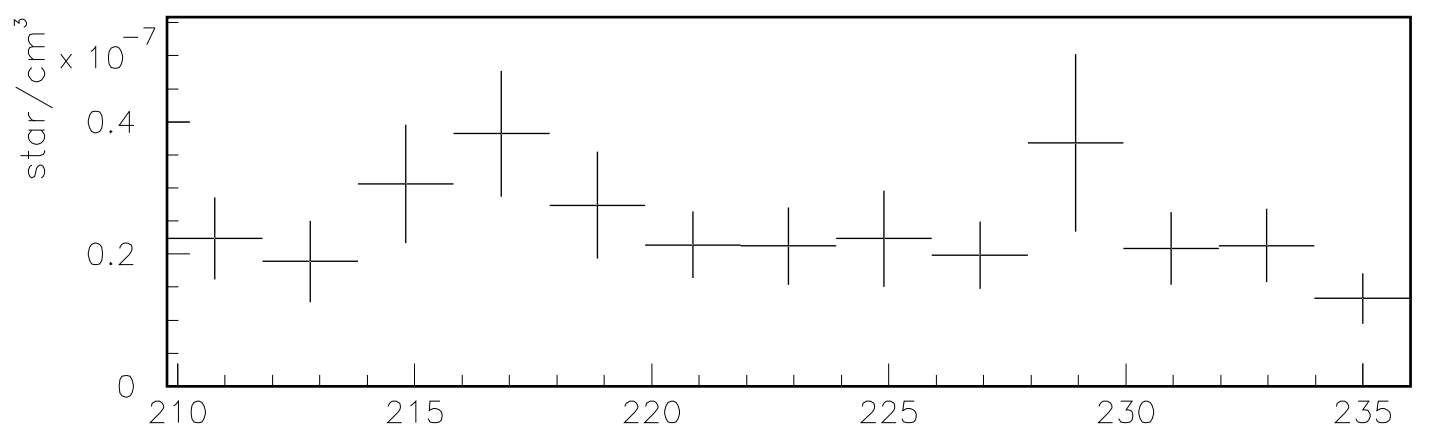

distance along tunnel in $m$

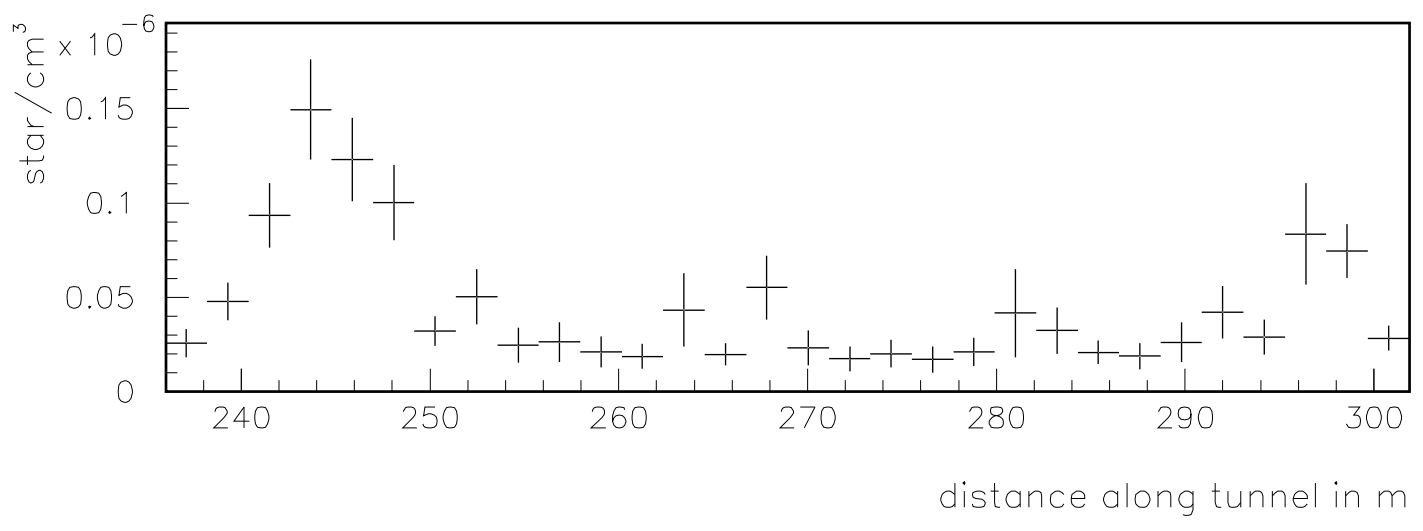

Star density distribution in $1 \mathrm{~cm}$ bin.

Figure B.9: Star density distribution for first three regions. Magnet current variation on $1.5 \%$ in HV101. 

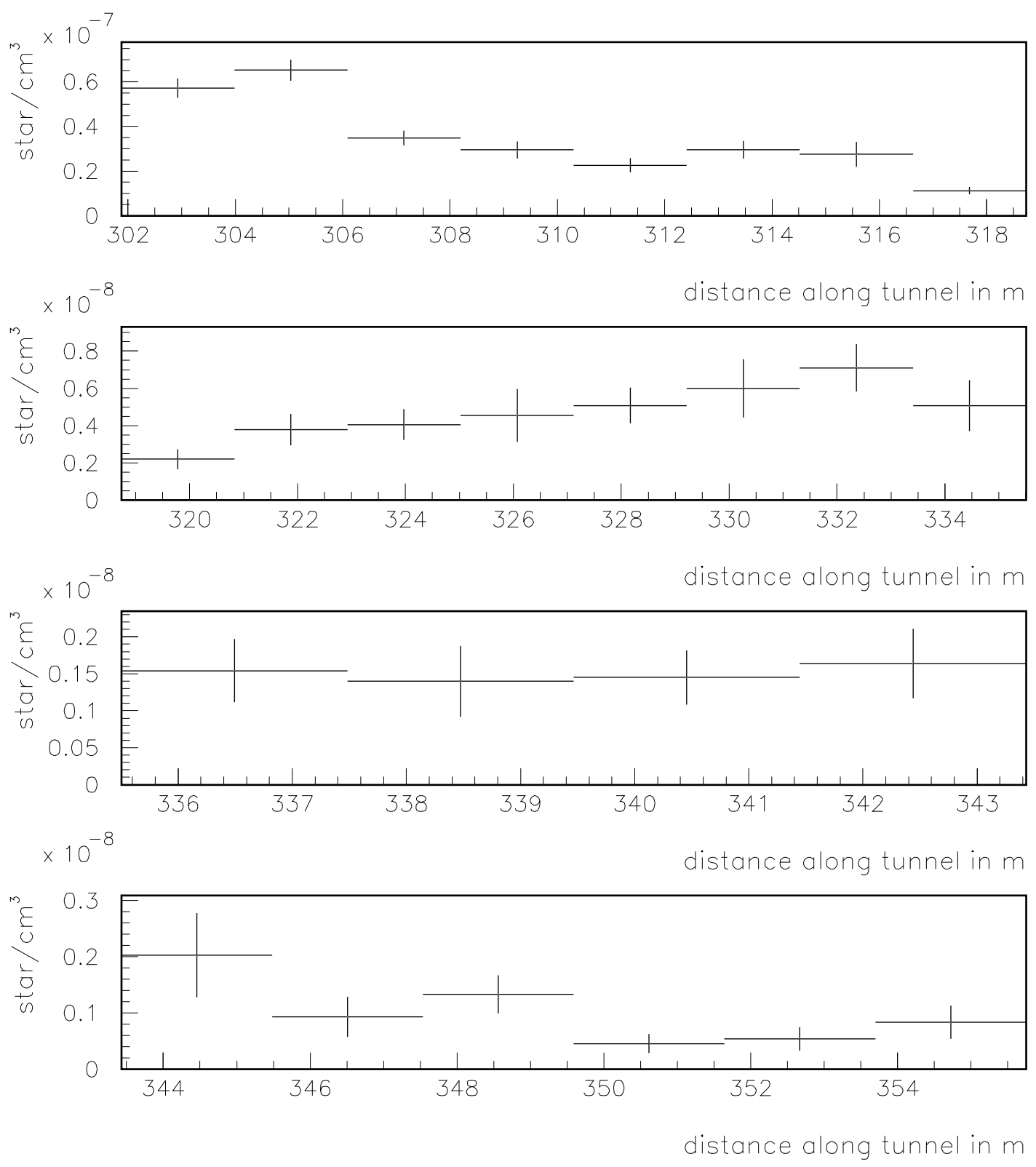

Star density distribution in $1 \mathrm{~cm}$ bin.

Figure B.10: Star density distribution for last four regions. Magnet current variation on $1.5 \%$ in HV101. 

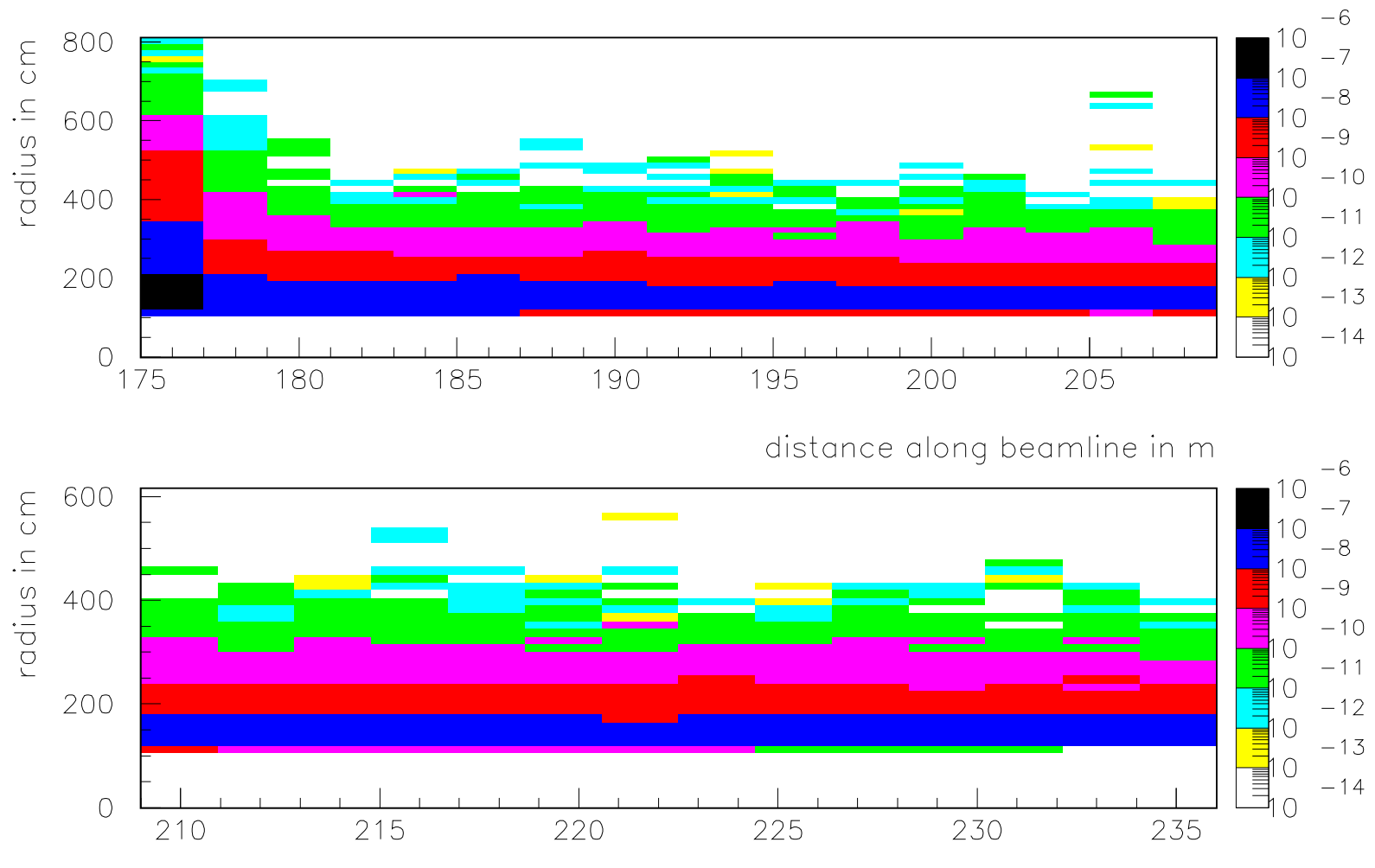

distance along beamline in $\mathrm{m}$

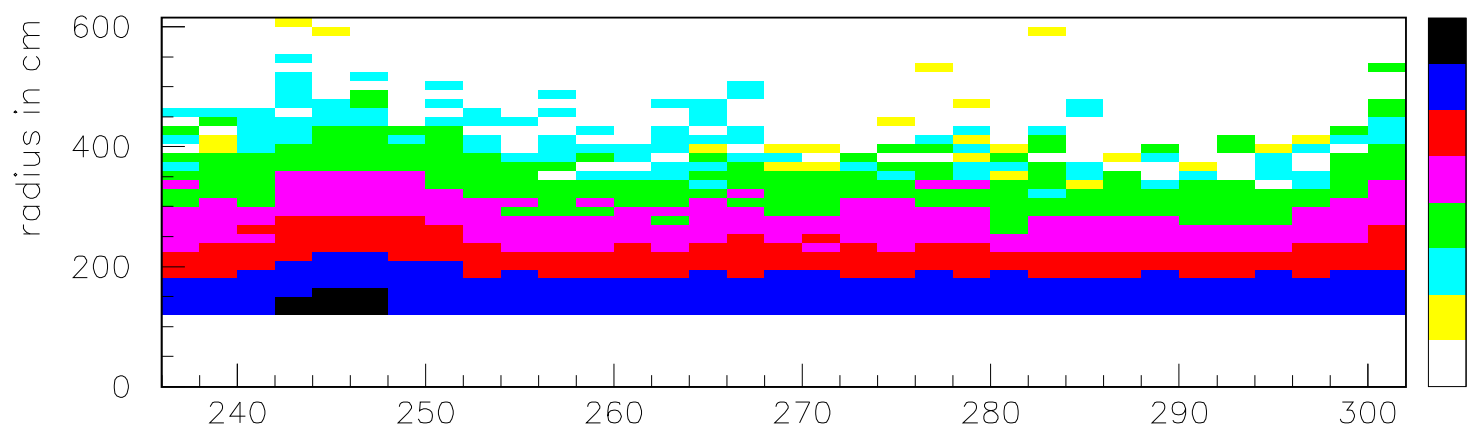

distance along beamline in $m$

Star density distribution. 1.5\% magnetic field variation in HV101

Figure B.11: Star density distribution for first three regions. 

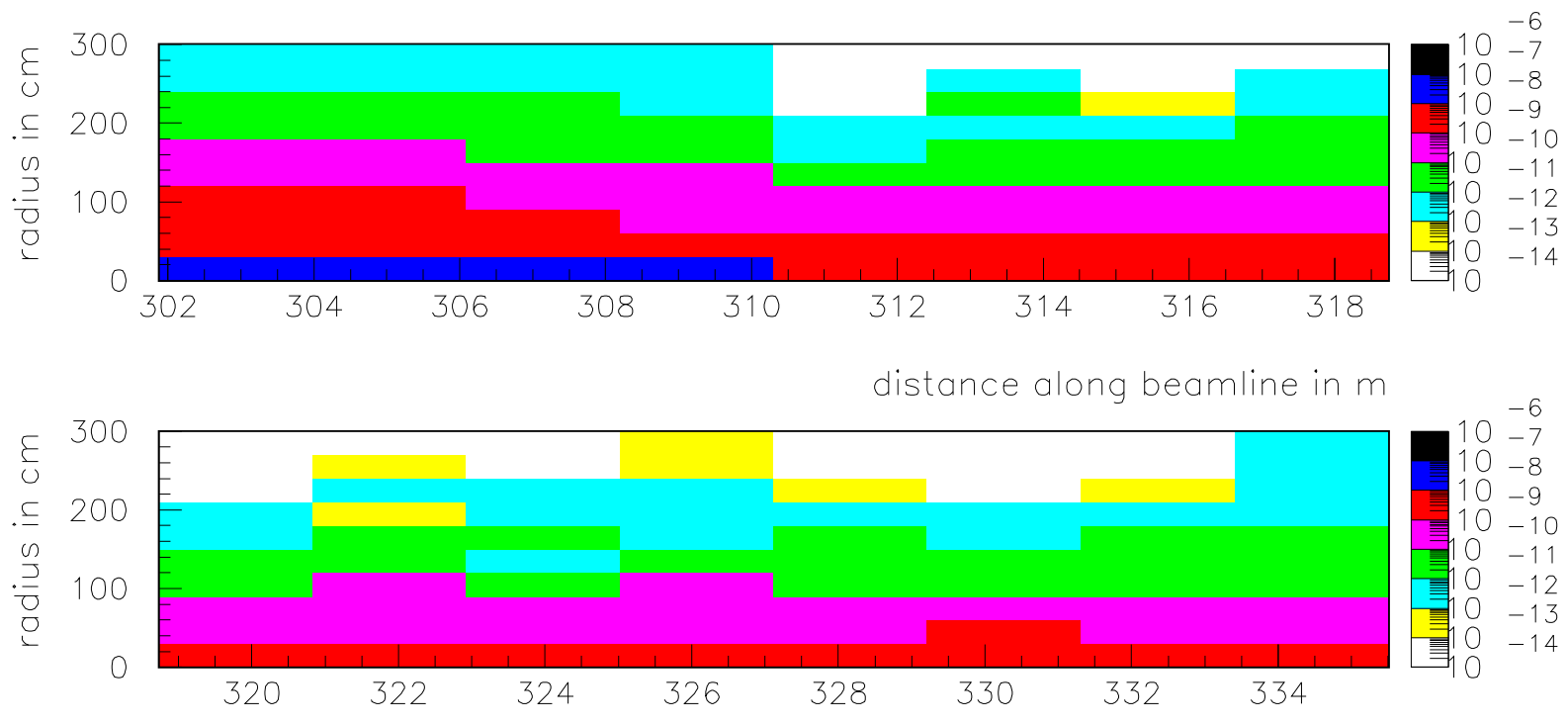

distance along beamline in $m$

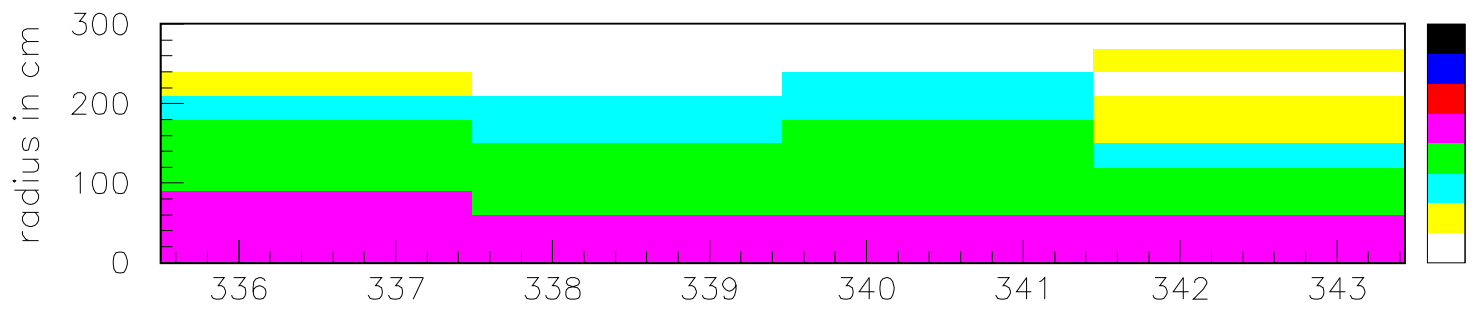

distance along beamline in $\mathrm{m}$

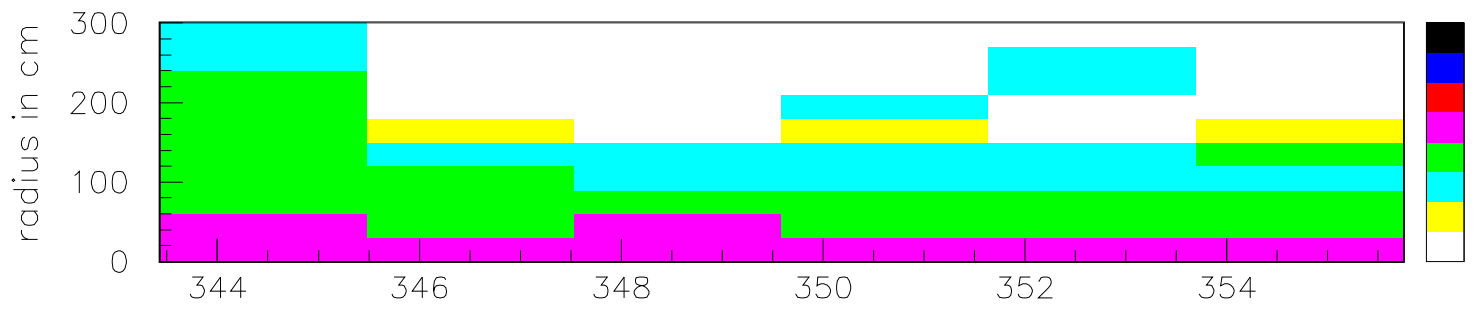

distance along beamline in $m$

Star density distribution. 1.5\% magnetic field variation in HV101

Figure B.12: Star density distribution for last four regions. 

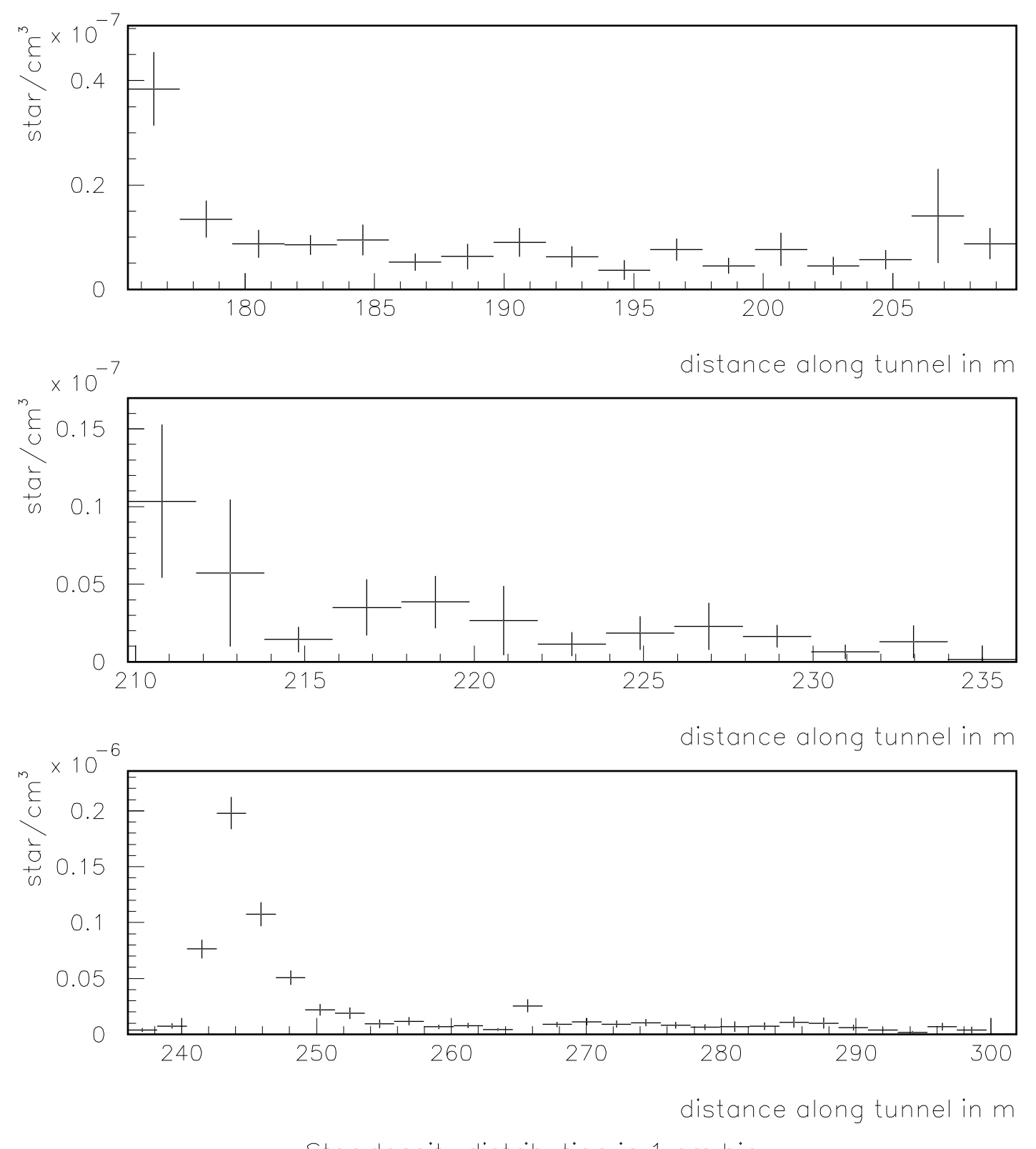

Star density distribution in $1 \mathrm{~cm}$ bin.

Figure B.13: Star density distribution for first three regions. Magnet current variation on $0.75 \%$ in V108. 

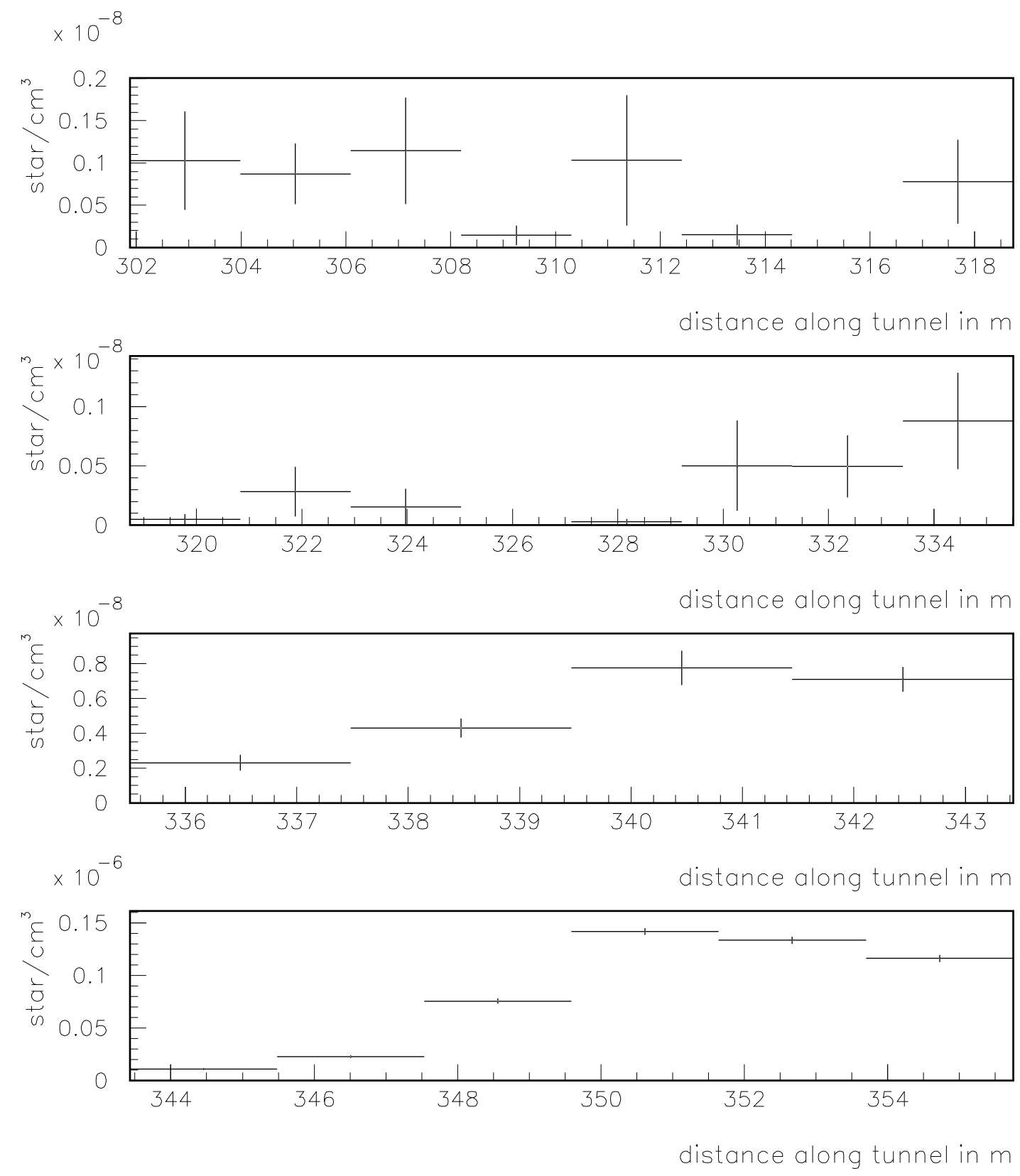

Star density distribution in $1 \mathrm{~cm}$ bin.

Figure B.14: Star density distribution for last four regions. Magnet current variation on $0.75 \%$ in $\mathrm{V} 108$. 

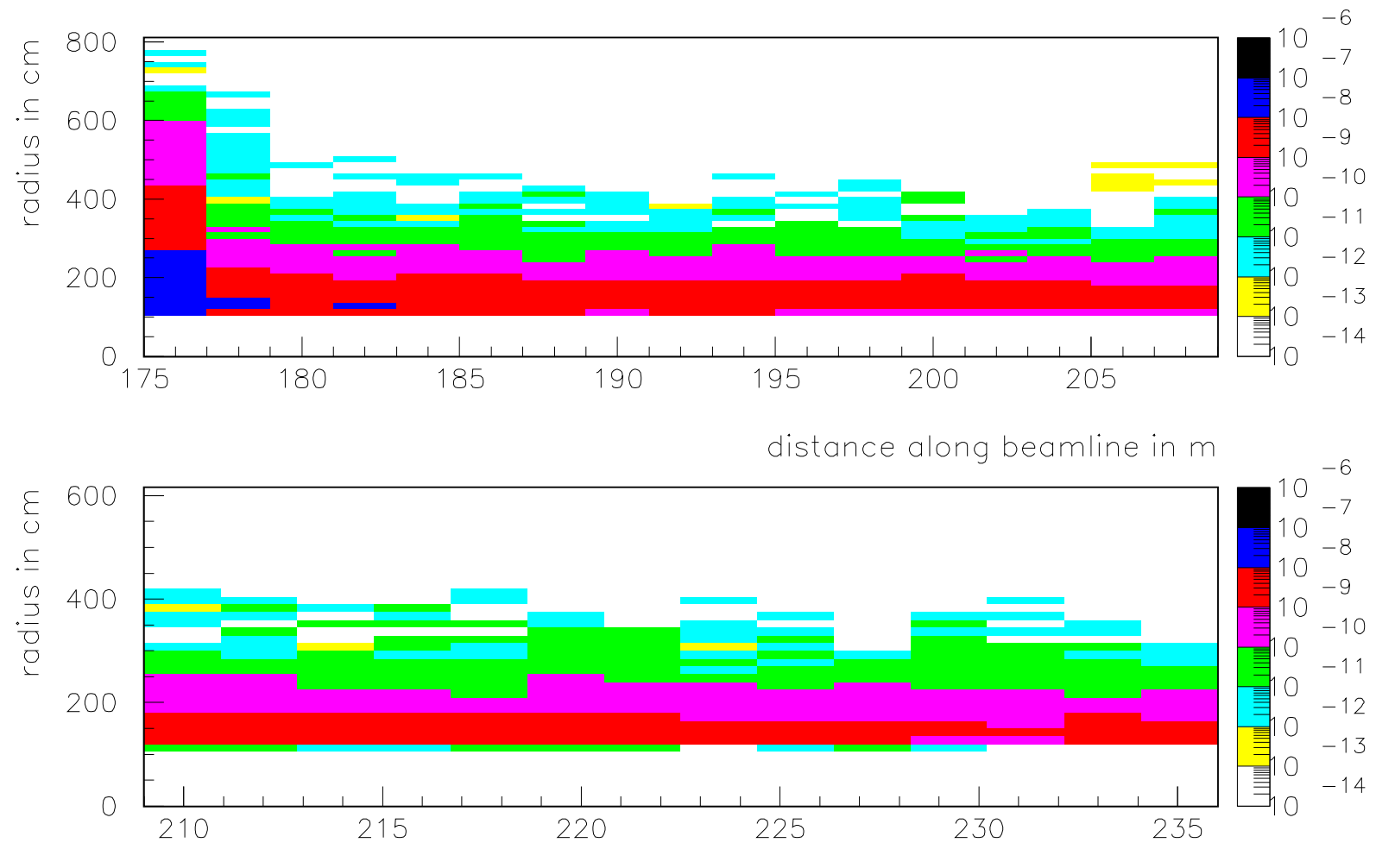

distance along beamline in $m$

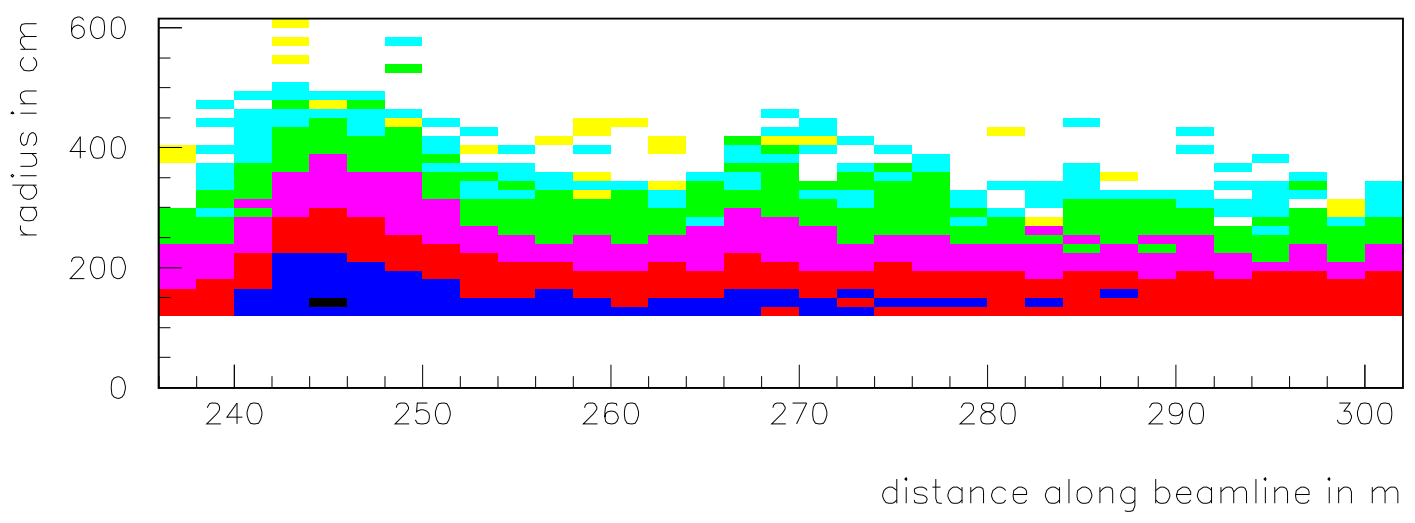

Star density. $0.75 \%$ magnetic field variation in V108

Figure B.15: Star density distribution for first three regions. 

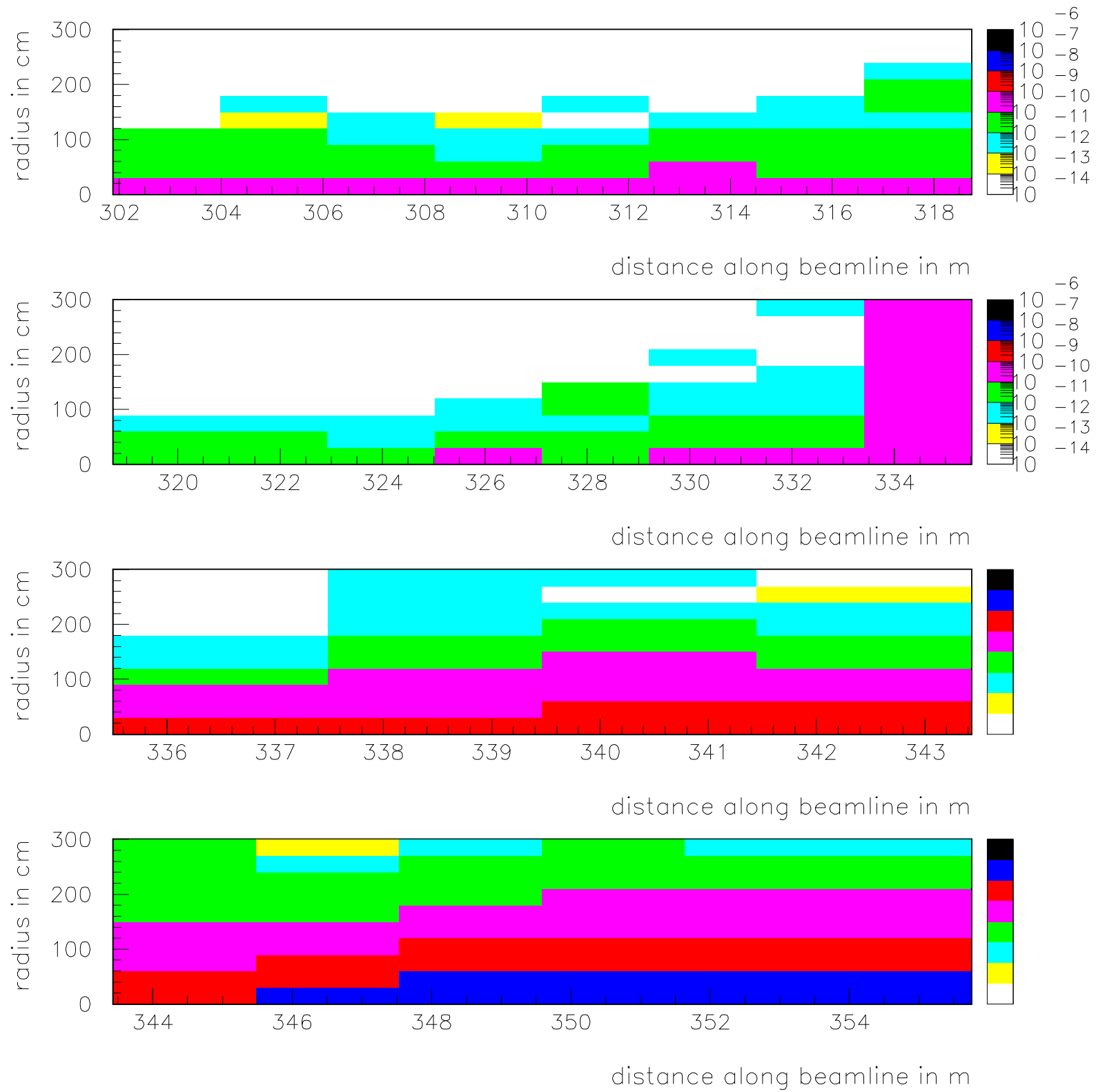

Star density. $0.75 \%$ magnetic field variation in $\mathrm{V} 108$

Figure B.16: Star density distribution for last four regions. 

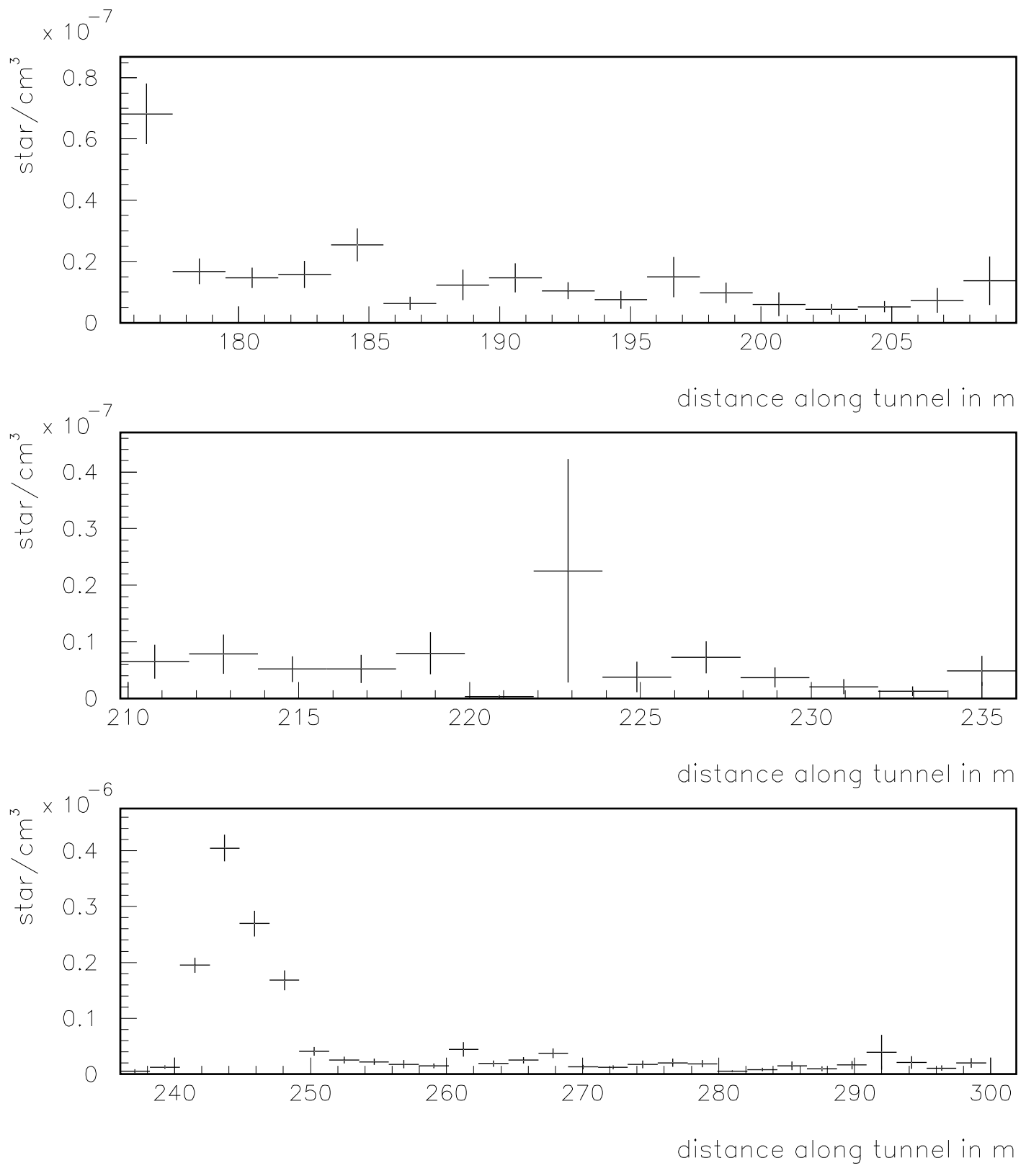

Star density distribution in $1 \mathrm{~cm}$ bin.

Figure B.17: Star density distribution for first three regions. Magnet current variation on $0.78 \%$ in V108. 

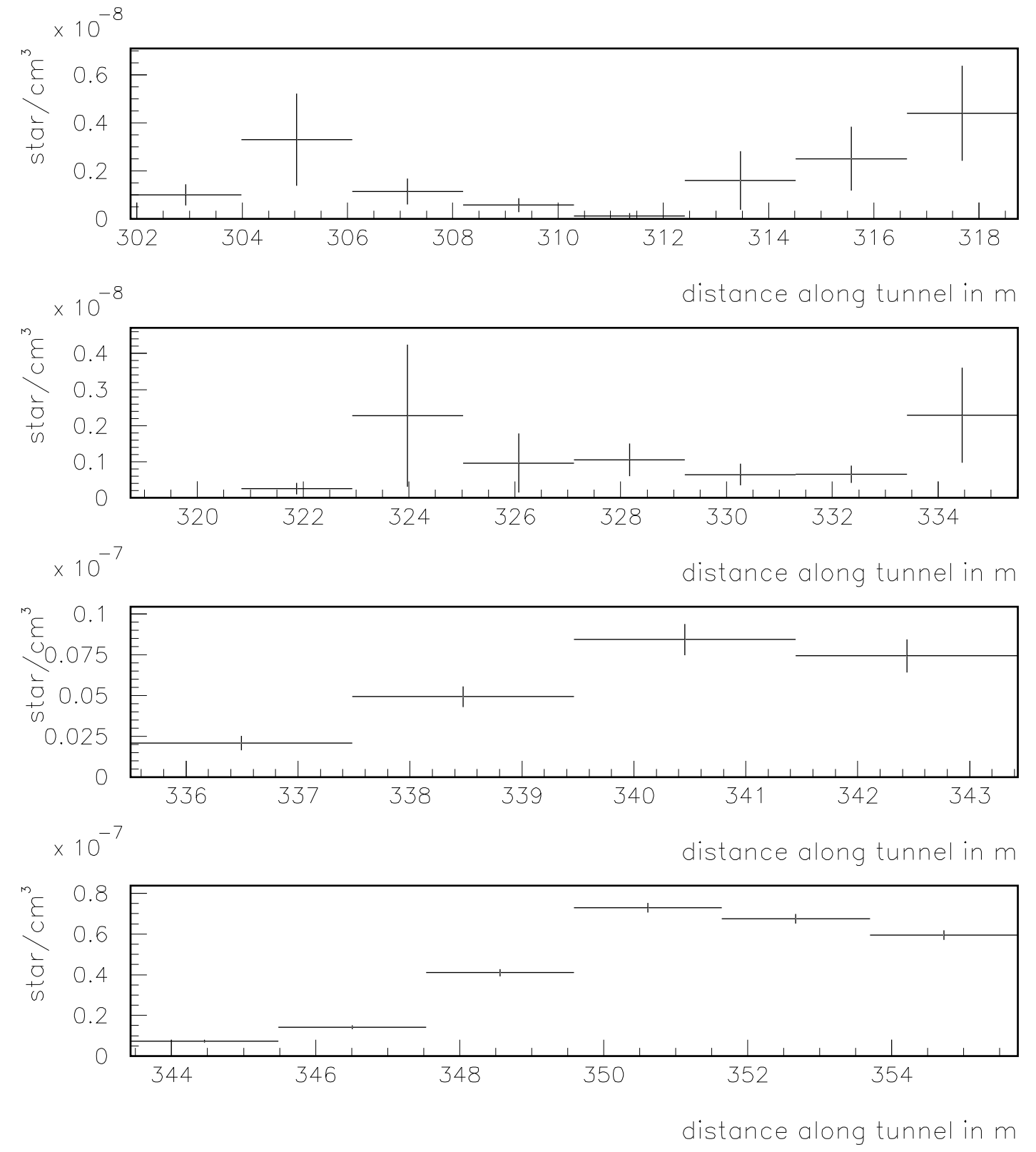

Star density distribution in $1 \mathrm{~cm}$ bin.

Figure B.18: Star density distribution for last four regions. Magnet current variation on $0.78 \%$ in $\mathrm{V} 108$. 

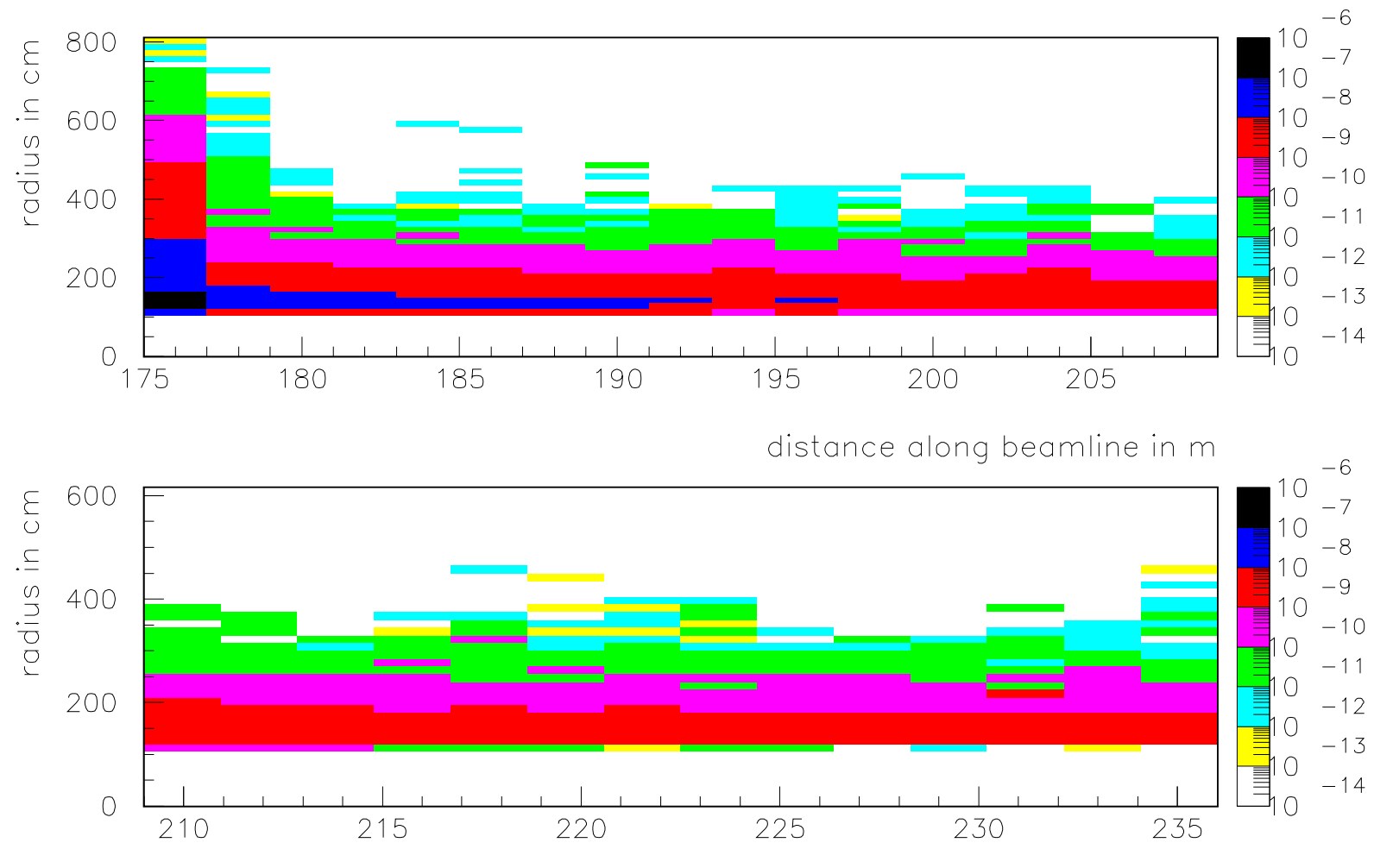

distance along beamline in $\mathrm{m}$

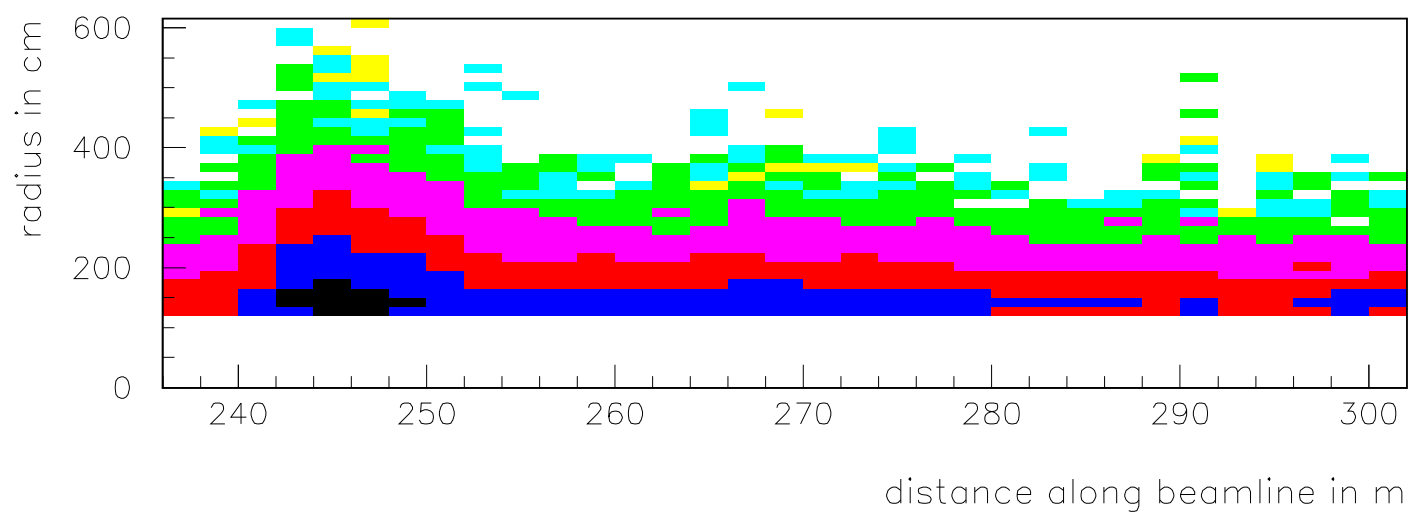

Star density. $0.78 \%$ magnetic field variation in V108

Figure B.19: Star density distribution for first three regions. 

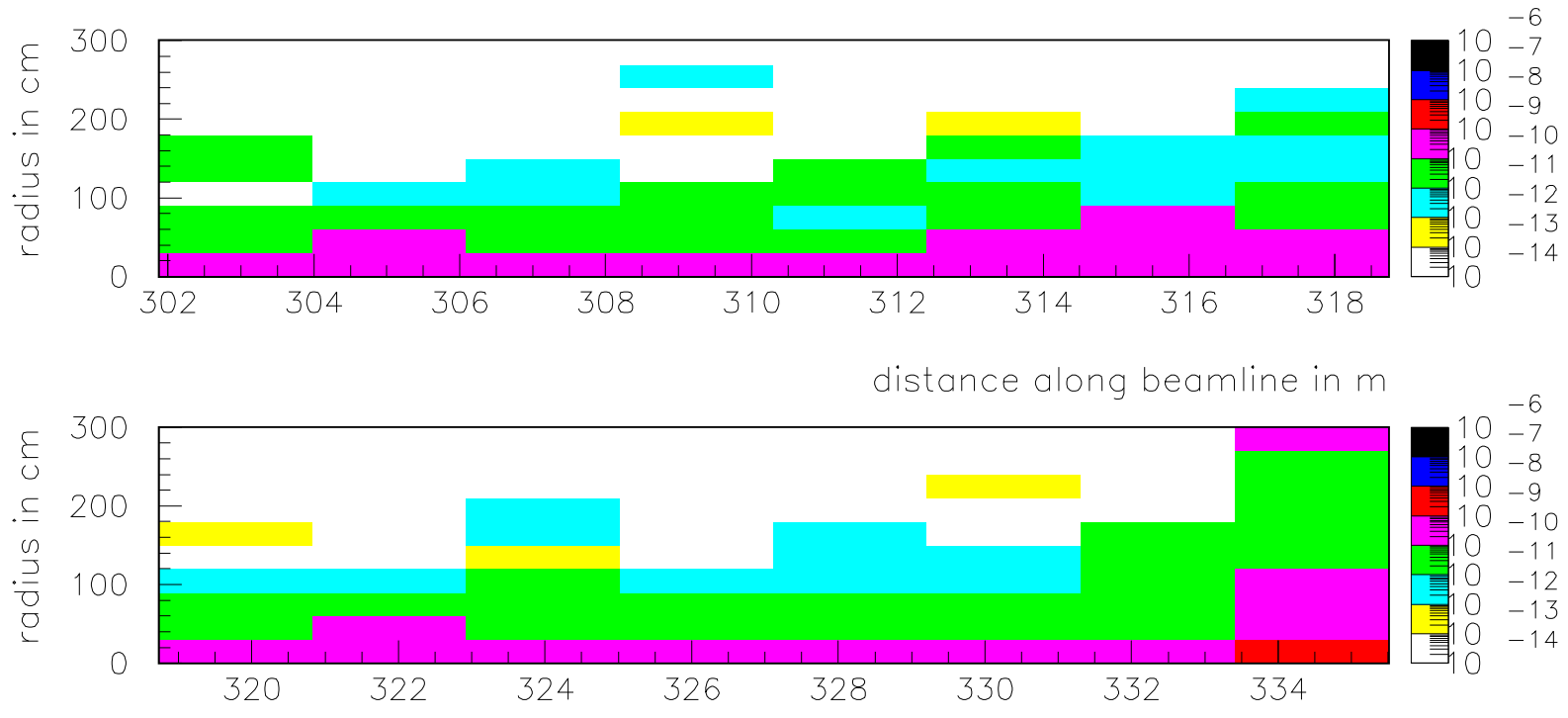

distance along beamline in $m$

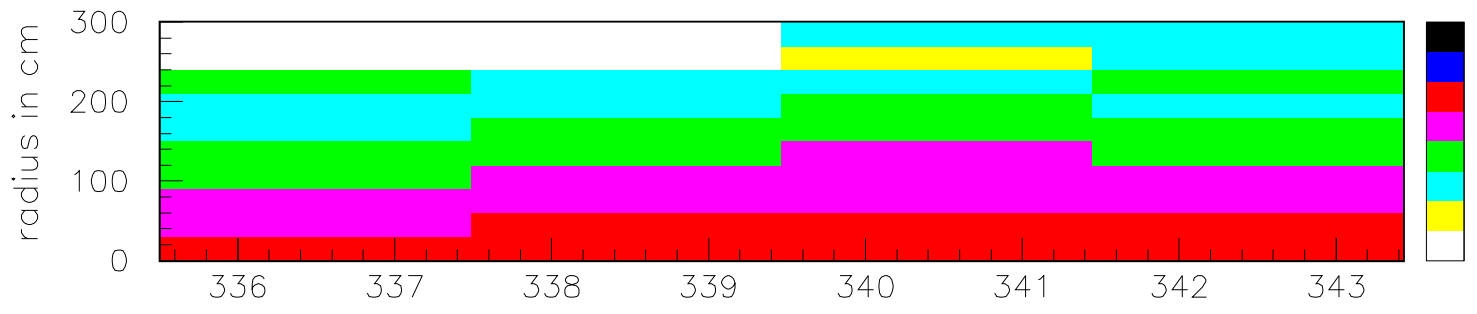

distance along beamline in $\mathrm{m}$

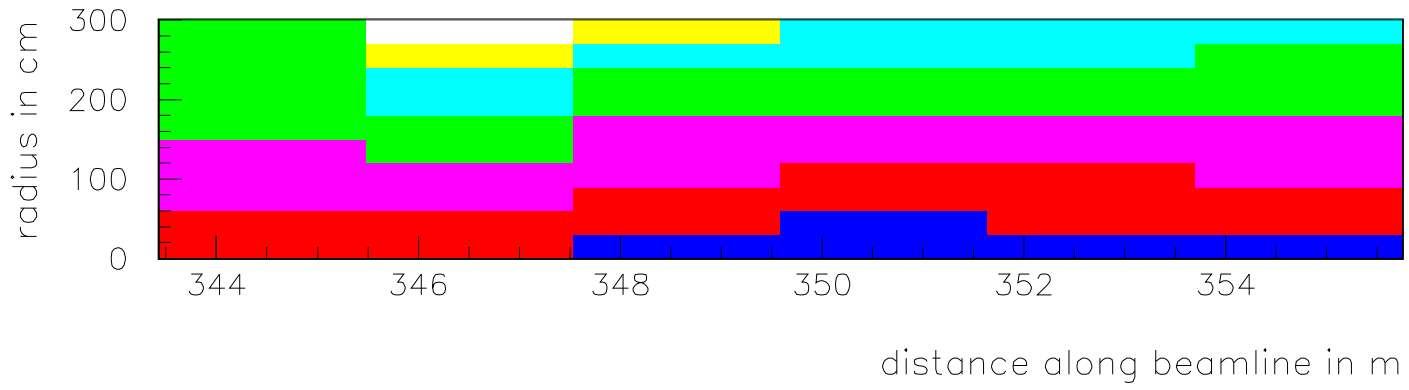

Star density. $0.78 \%$ magnetic field variation in V108

Figure B.20: Star density distribution for last four regions. 

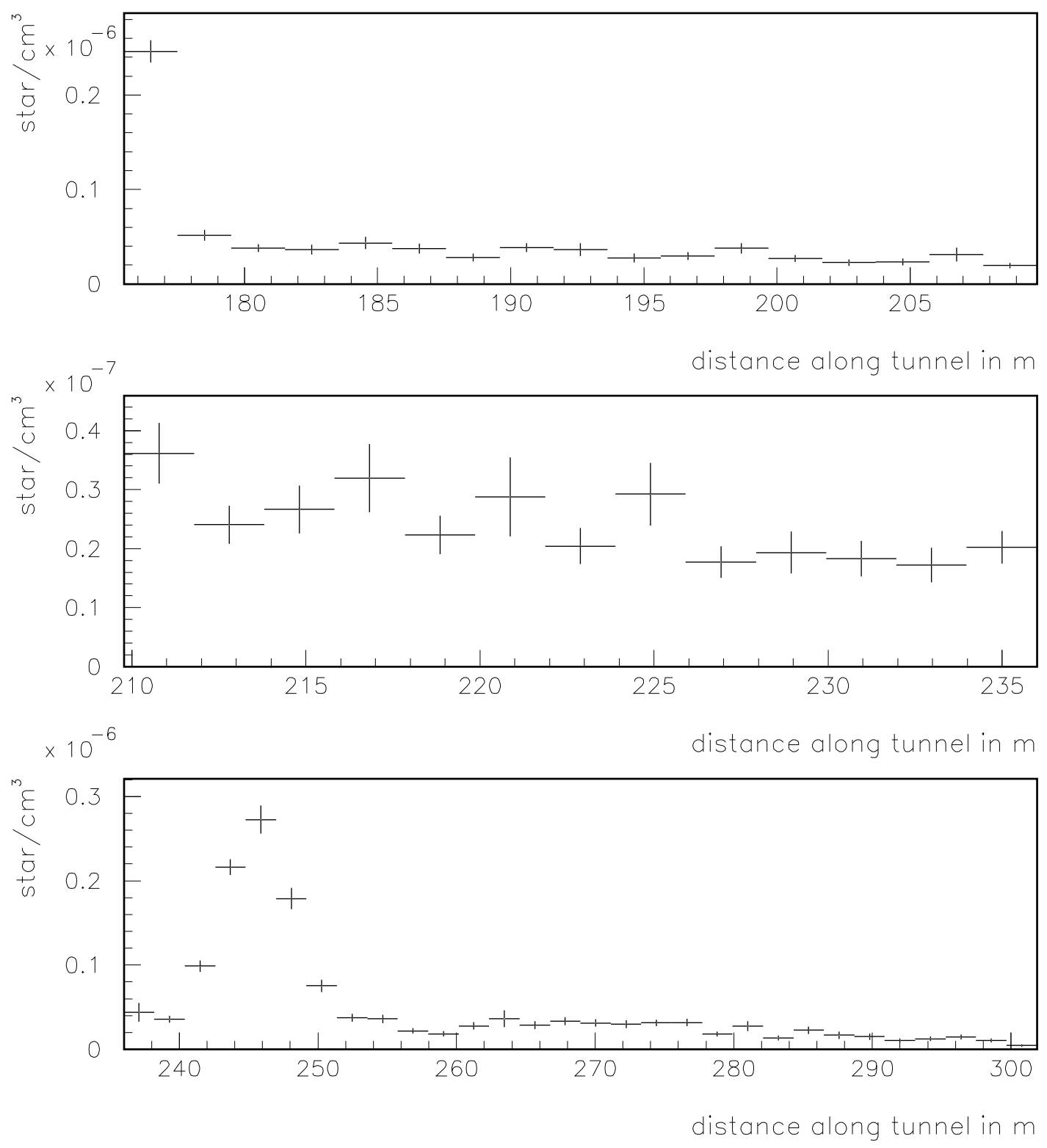

Star density distribution in $1 \mathrm{~cm}$ bin.

Figure B.21: Star density distribution for first three regions. Magnet current variation on $0.85 \%$ in $\mathrm{V} 108$. 

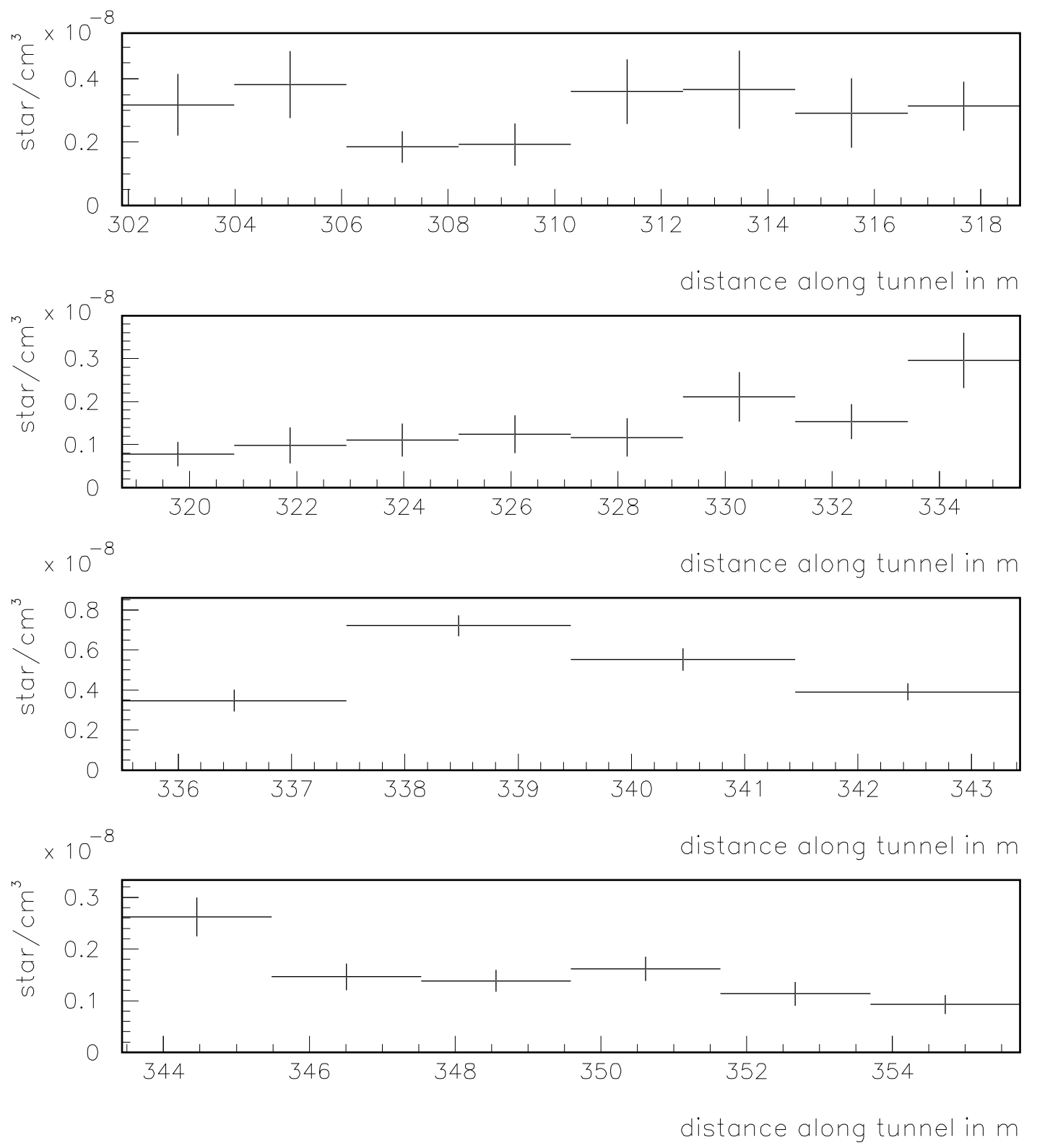

Star density distribution in $1 \mathrm{~cm}$ bin.

Figure B.22: Star density distribution for last four regions. Magnet current variation on $0.85 \%$ in $\mathrm{V} 108$. 

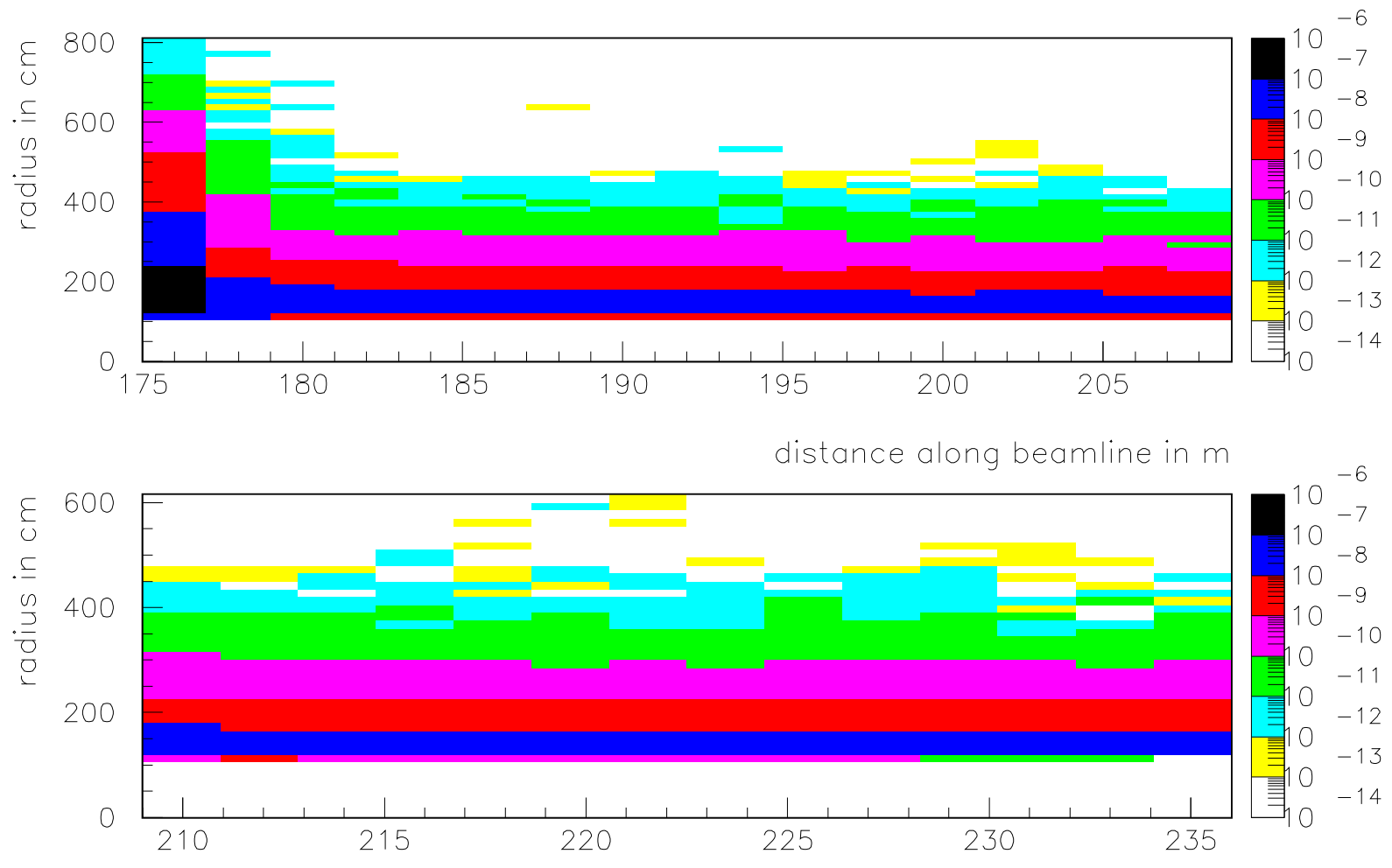

distance along beamline in $\mathrm{m}$

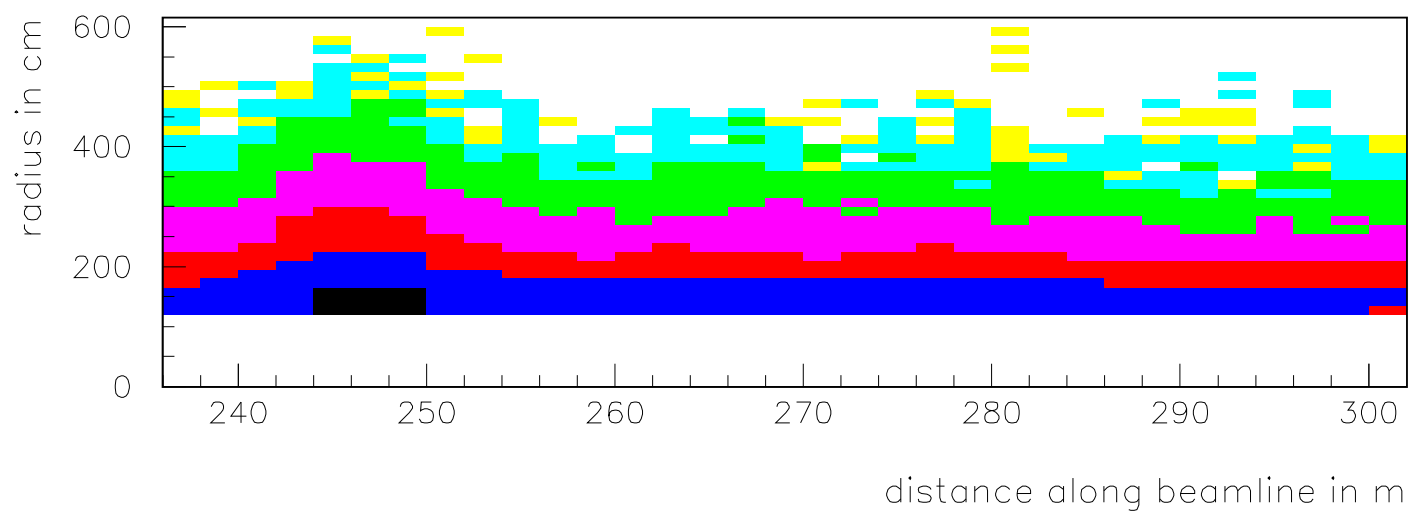

Star density. $0.85 \%$ magnetic field variation in V108

Figure B.23: Star density distribution for first three regions. 

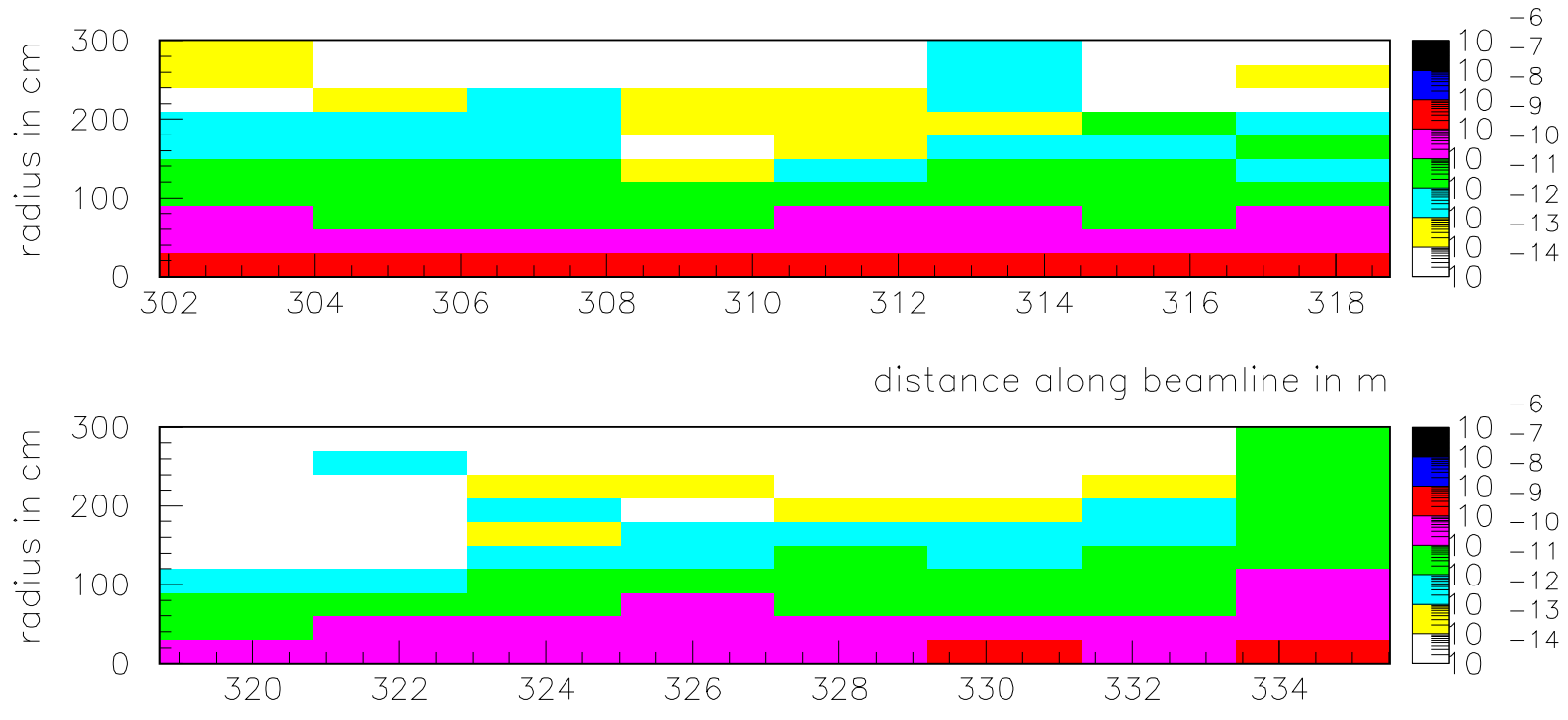

distance along beamline in $m$

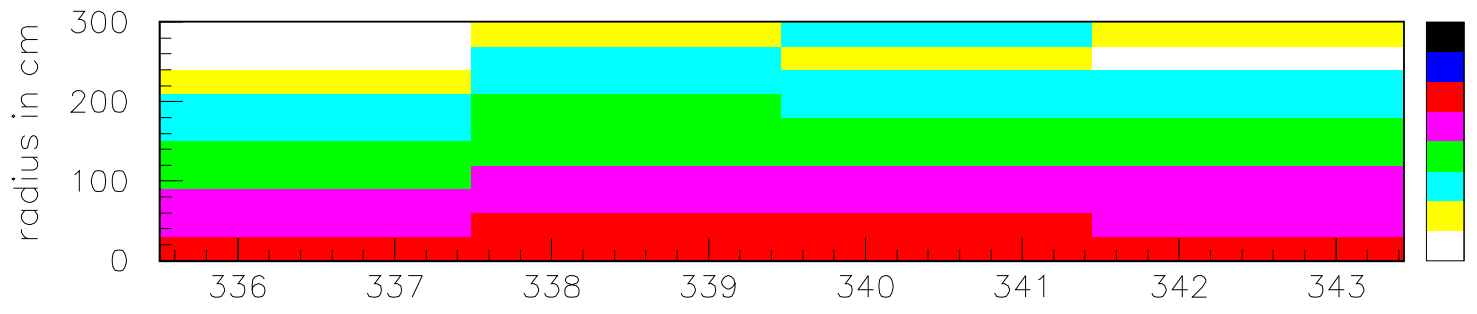

distance along beamline in $\mathrm{m}$

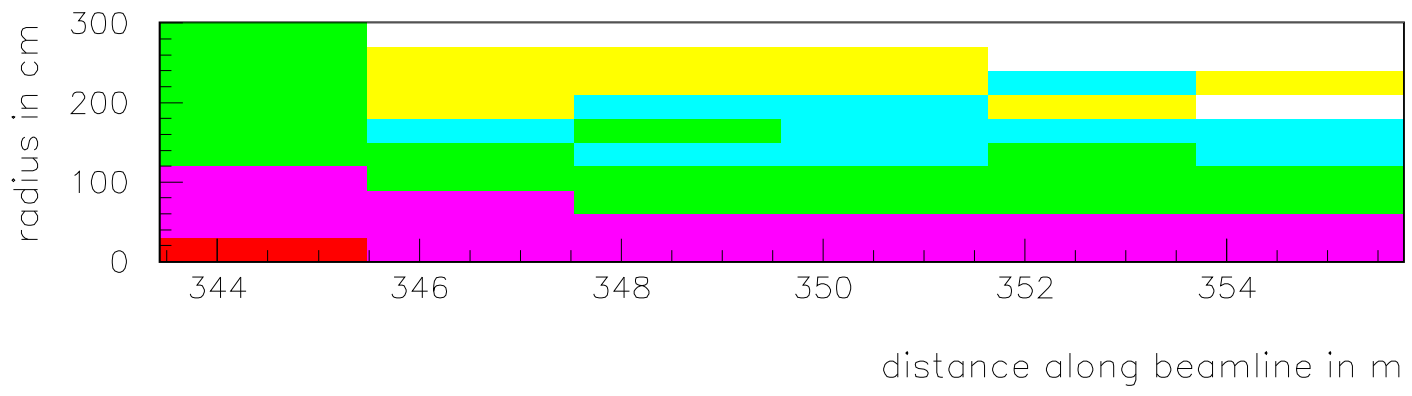

Star density. $0.85 \%$ magnetic field variation in V108

Figure B.24: Star density distribution for last four regions. 

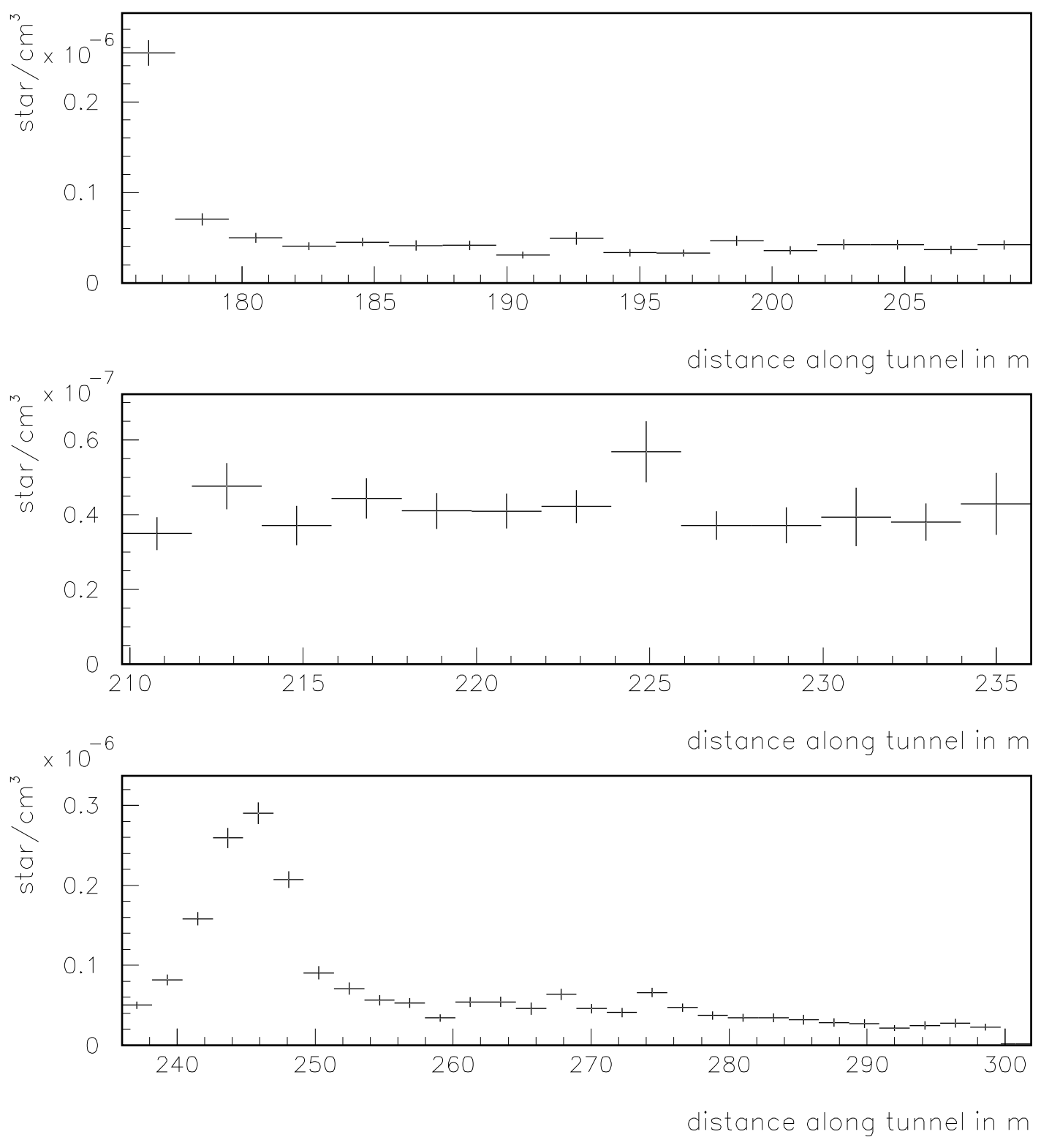

Star density distribution in $1 \mathrm{~cm}$ bin.

Figure B.25: Star density distribution for first three regions. Magnet current variation on $0.9 \%$ in V108. 

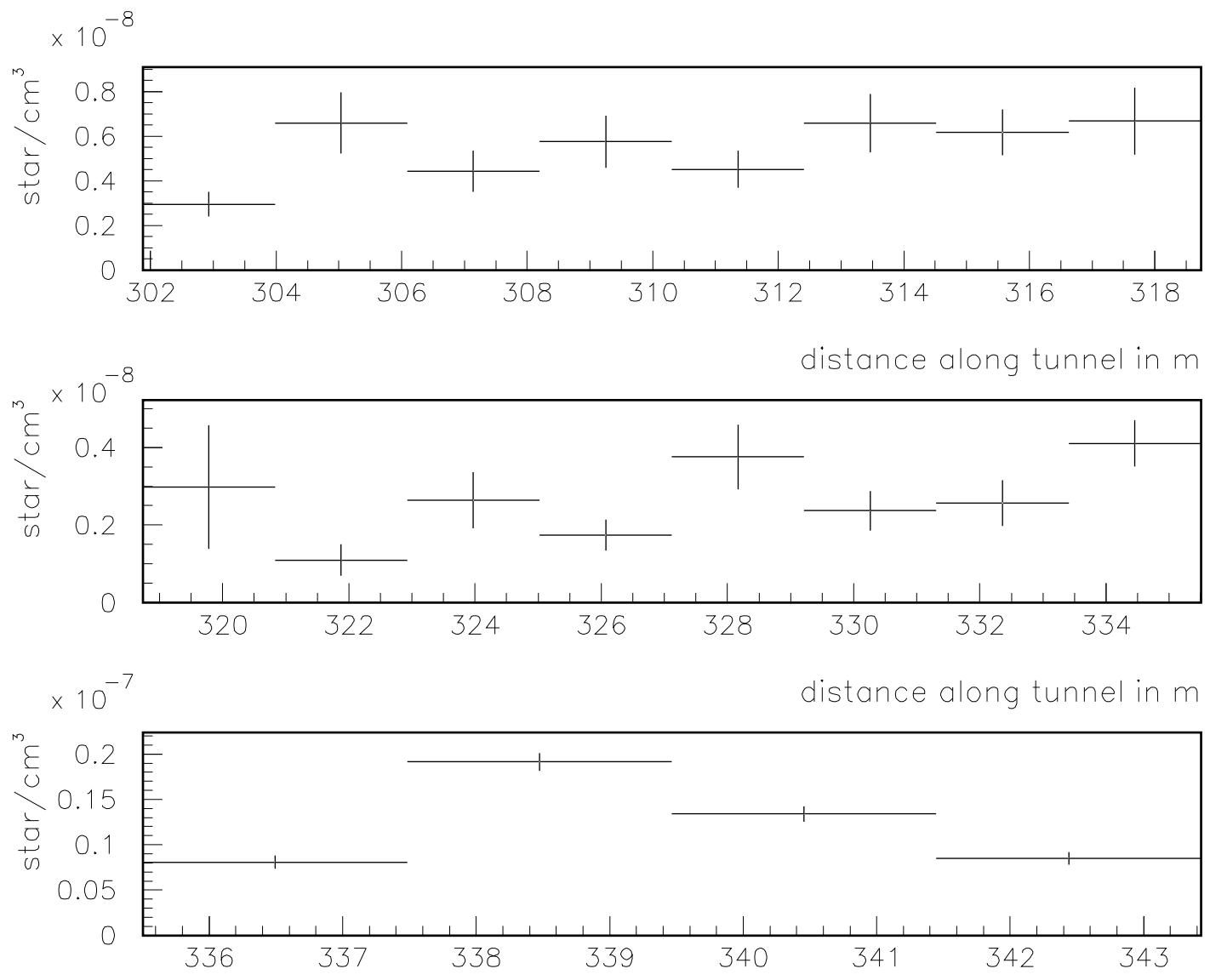

$$
\times 10^{-8}
$$

distance along tunnel in $m$

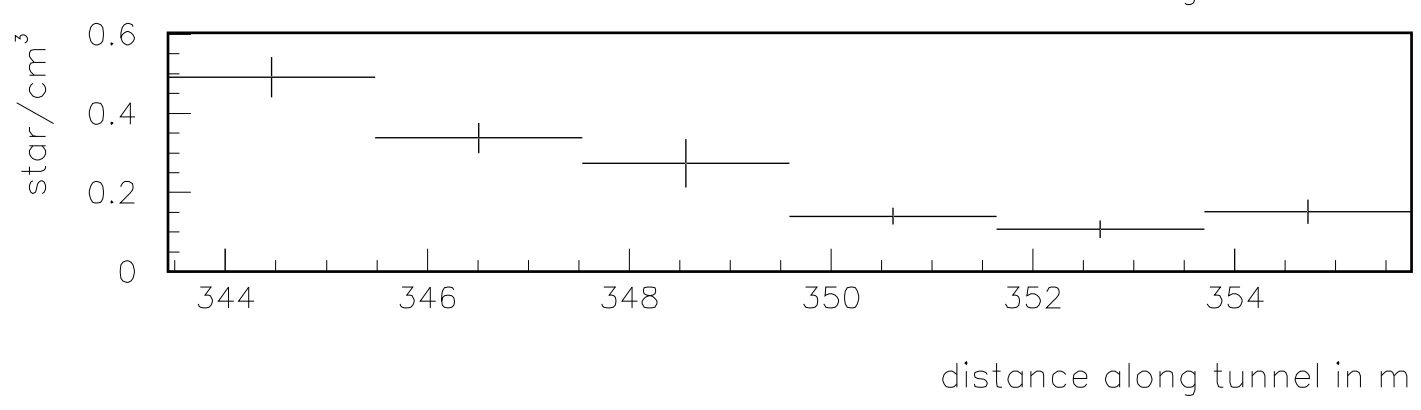

Star density distribution in $1 \mathrm{~cm}$ bin.

Figure B.26: Star density distribution for last four regions. Magnet current variation on $0.9 \%$ in V108. 

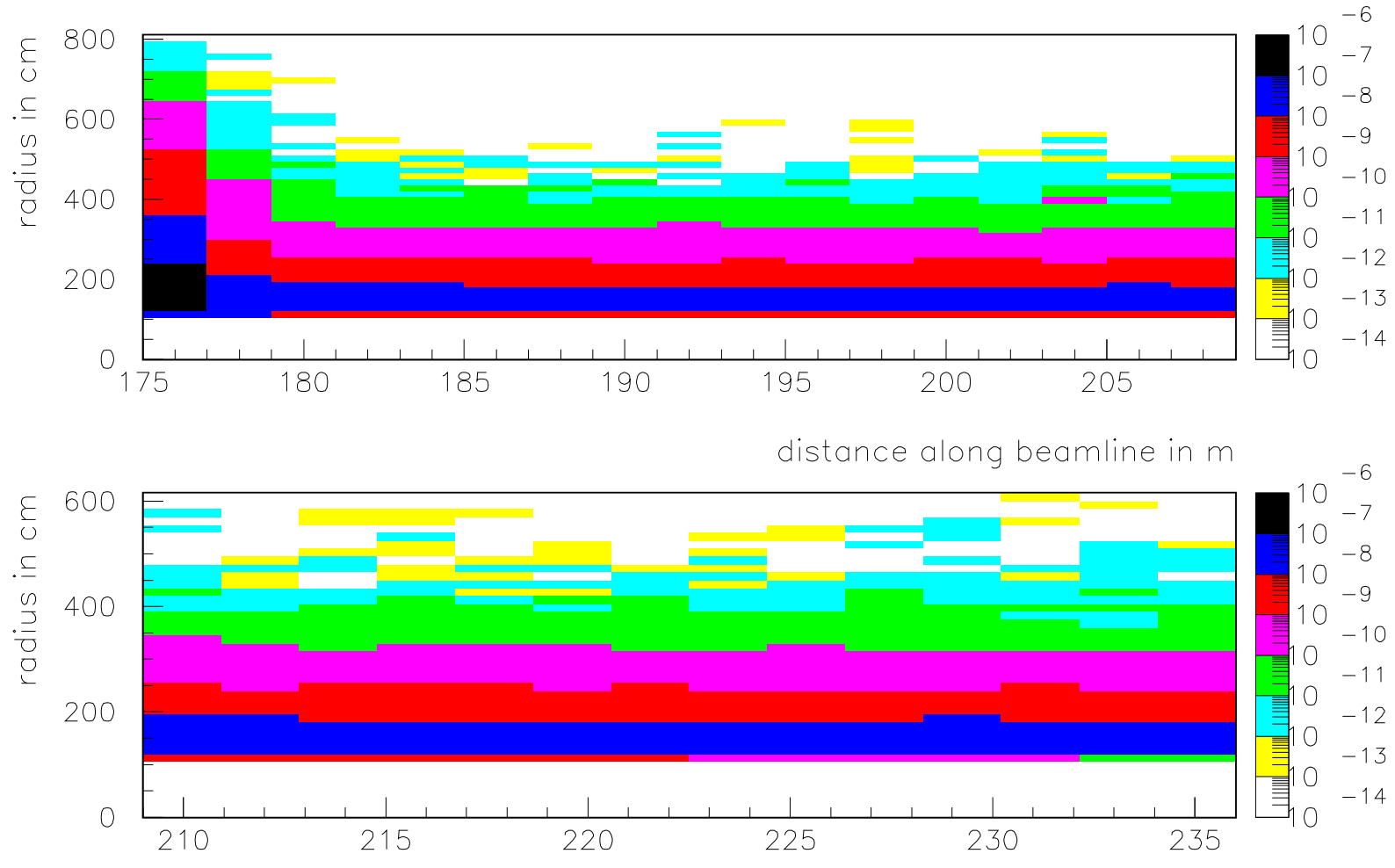

distance along beamline in $\mathrm{m}$

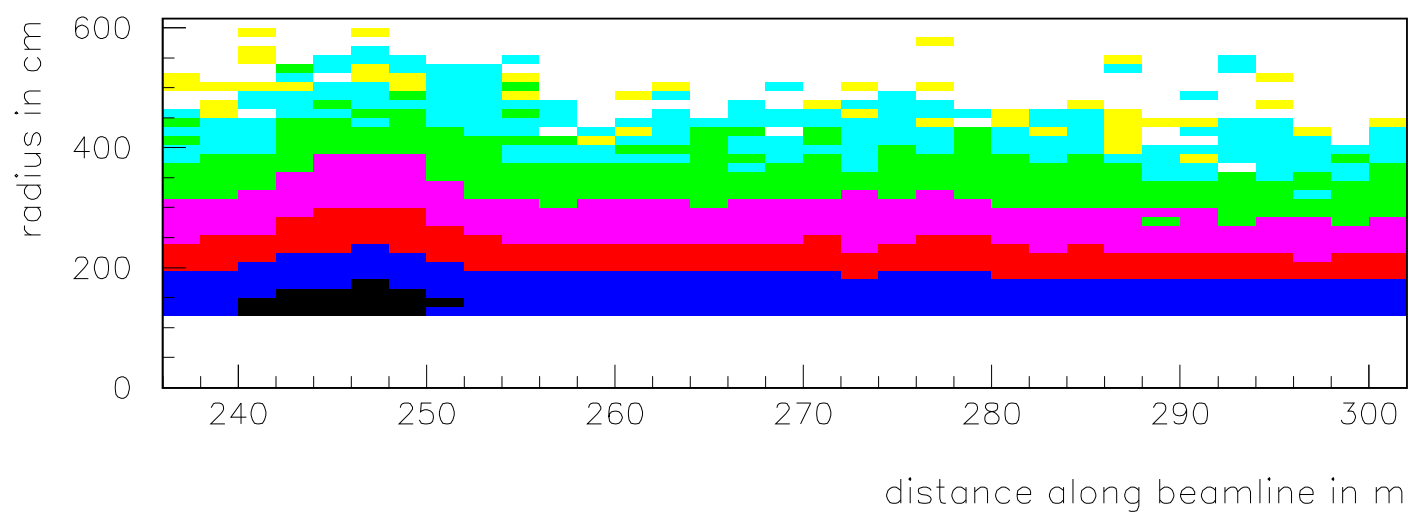

Star density. $0.9 \%$ magnetic field variation in V108

Figure B.27: Star density distribution for first three regions. 

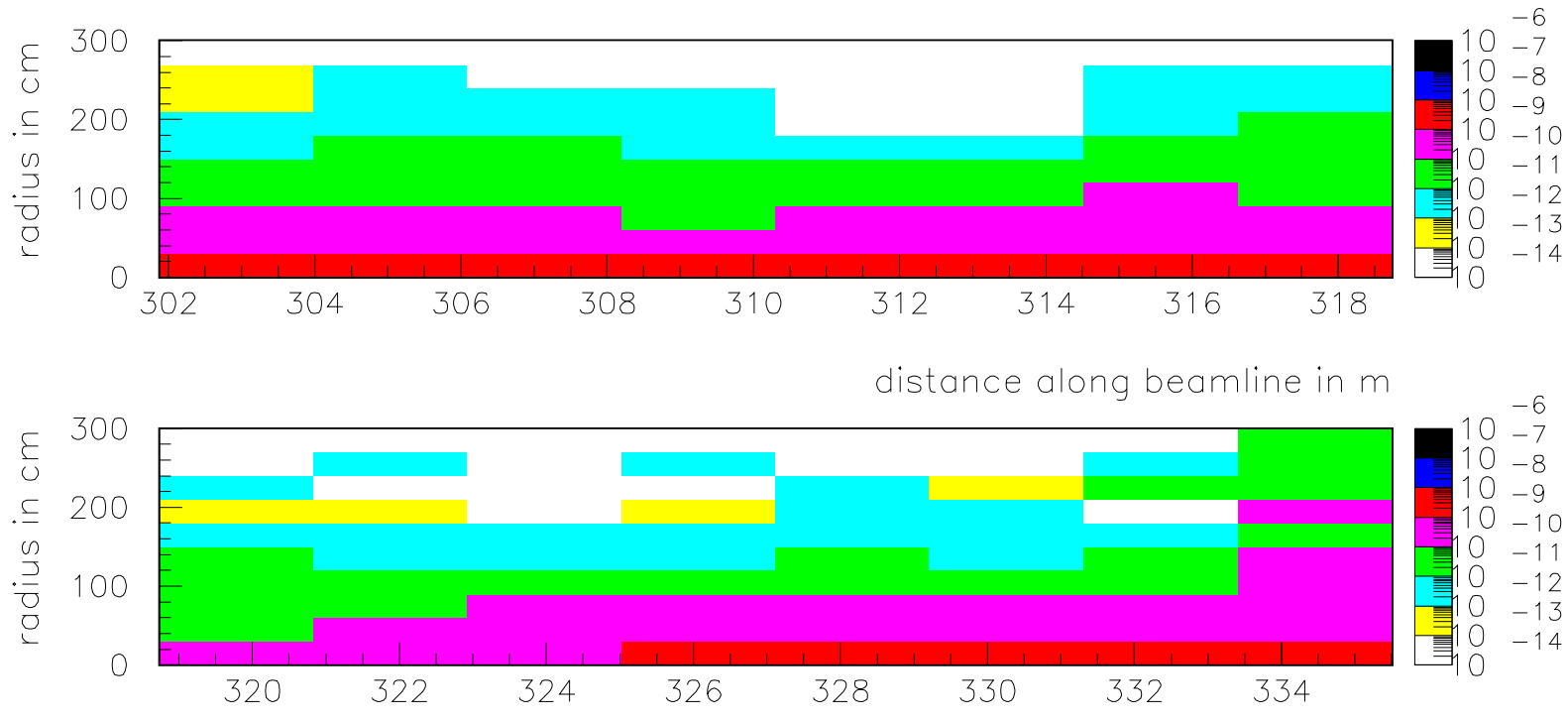

distance along beamline in $\mathrm{m}$

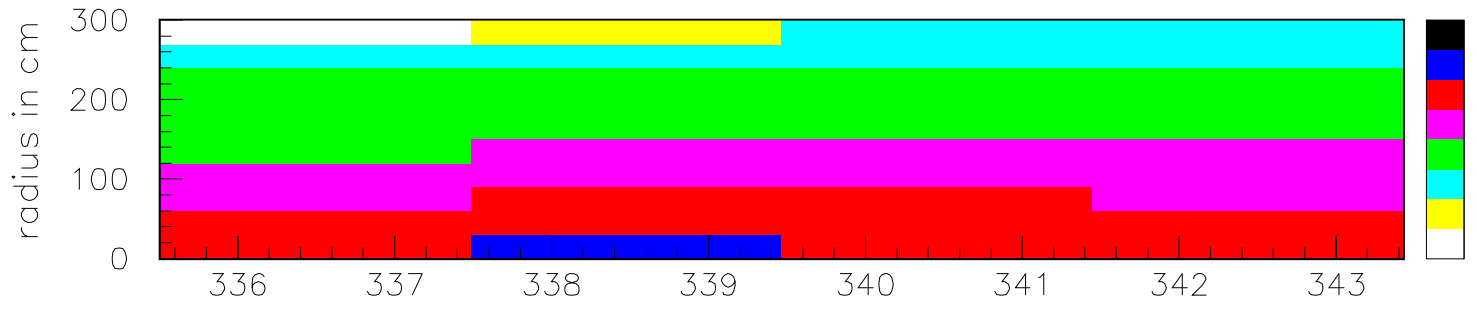

distance along beamline in $\mathrm{m}$

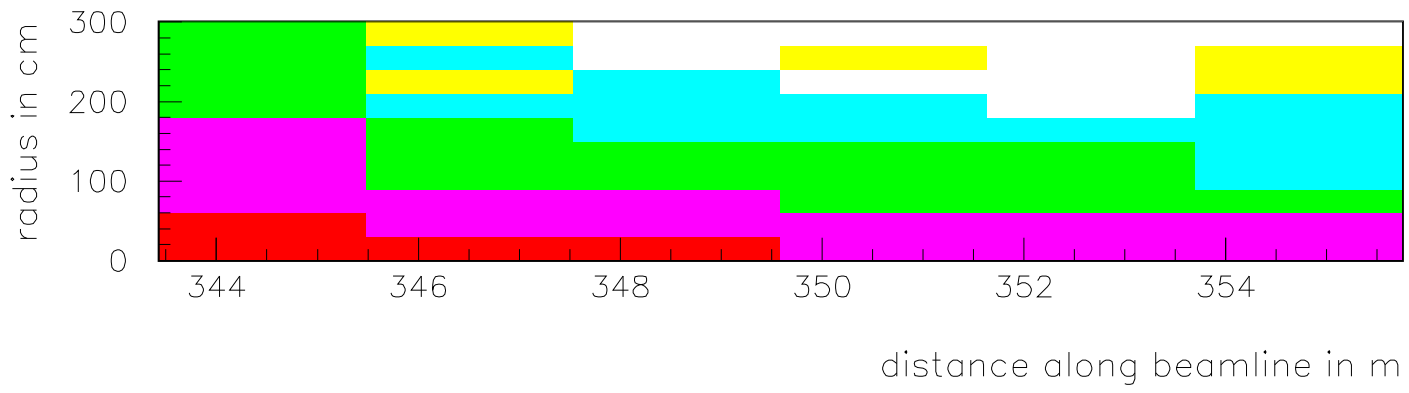

Star density. $0.9 \%$ magnetic field variation in V108

Figure B.28: Star density distribution for last four regions. 


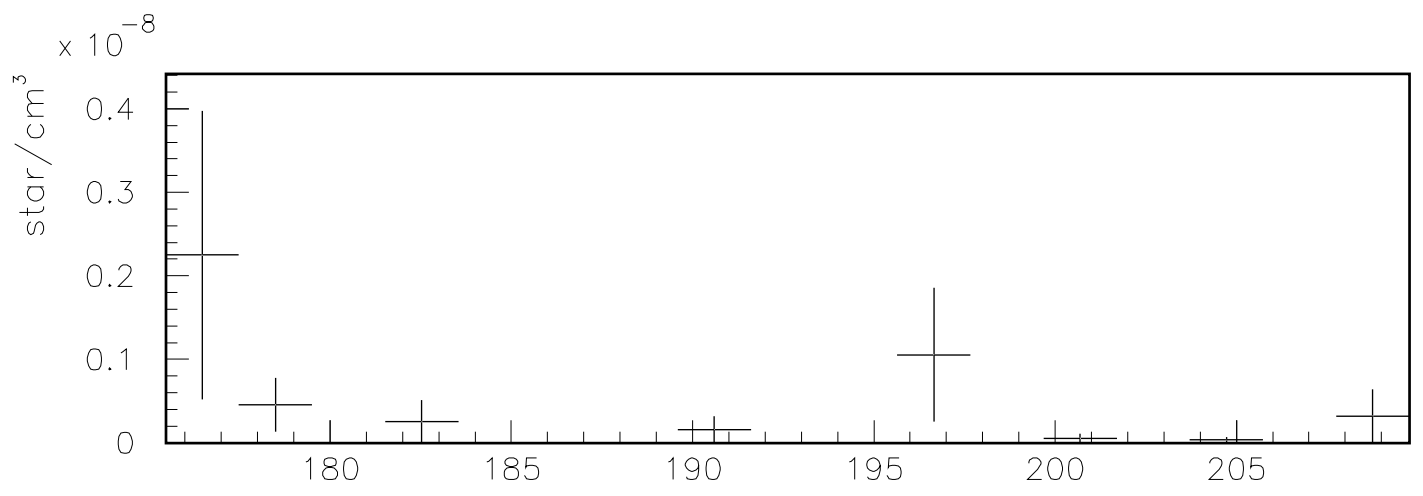

distance along tunnel in m
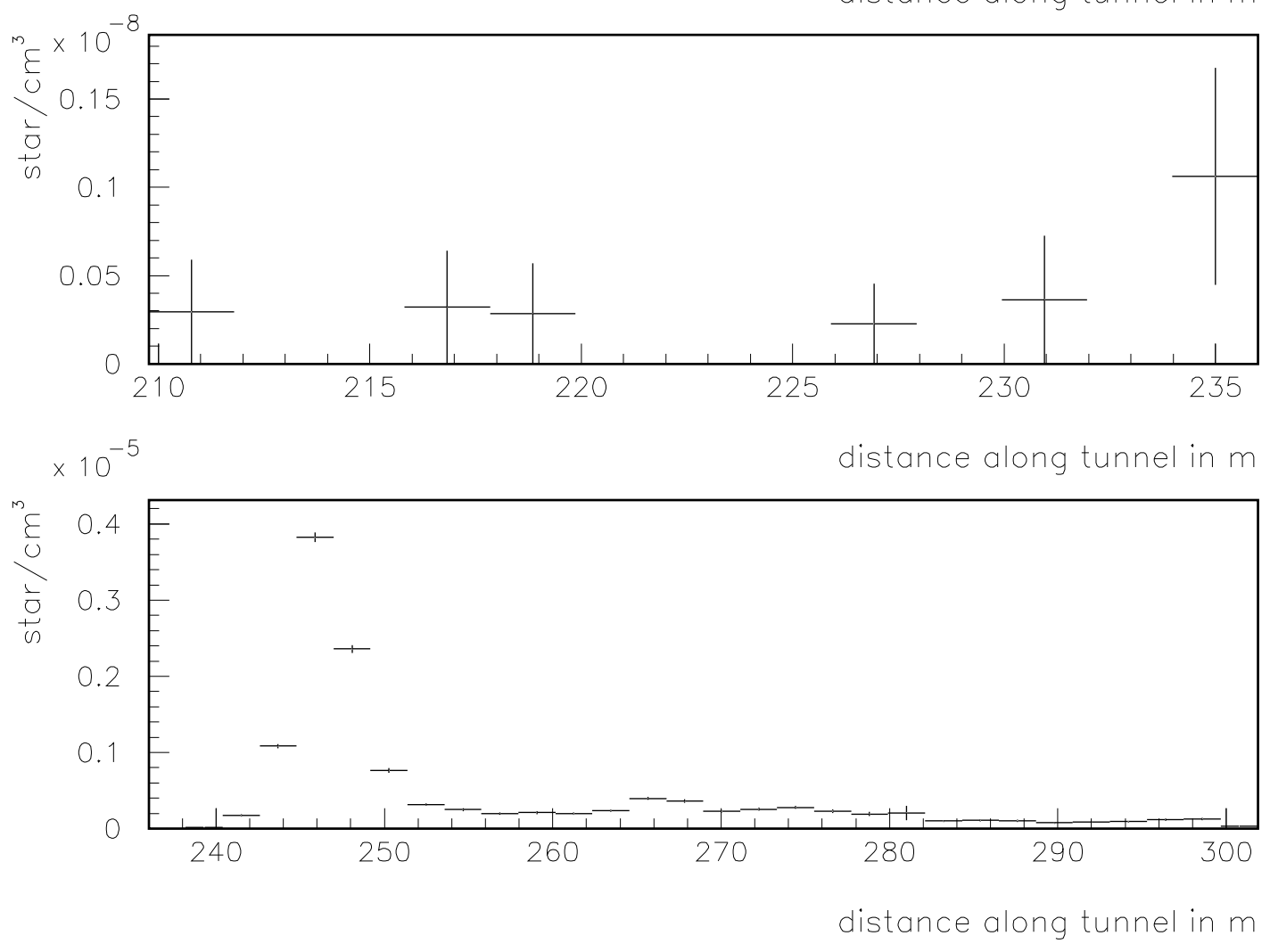

Star density distribution in $1 \mathrm{~cm}$ bin.

Figure B.29: Star density distribution for first three regions. Magnet current variation on $1.4 \%$ HV101 and $0.1 \%$ in V108. 

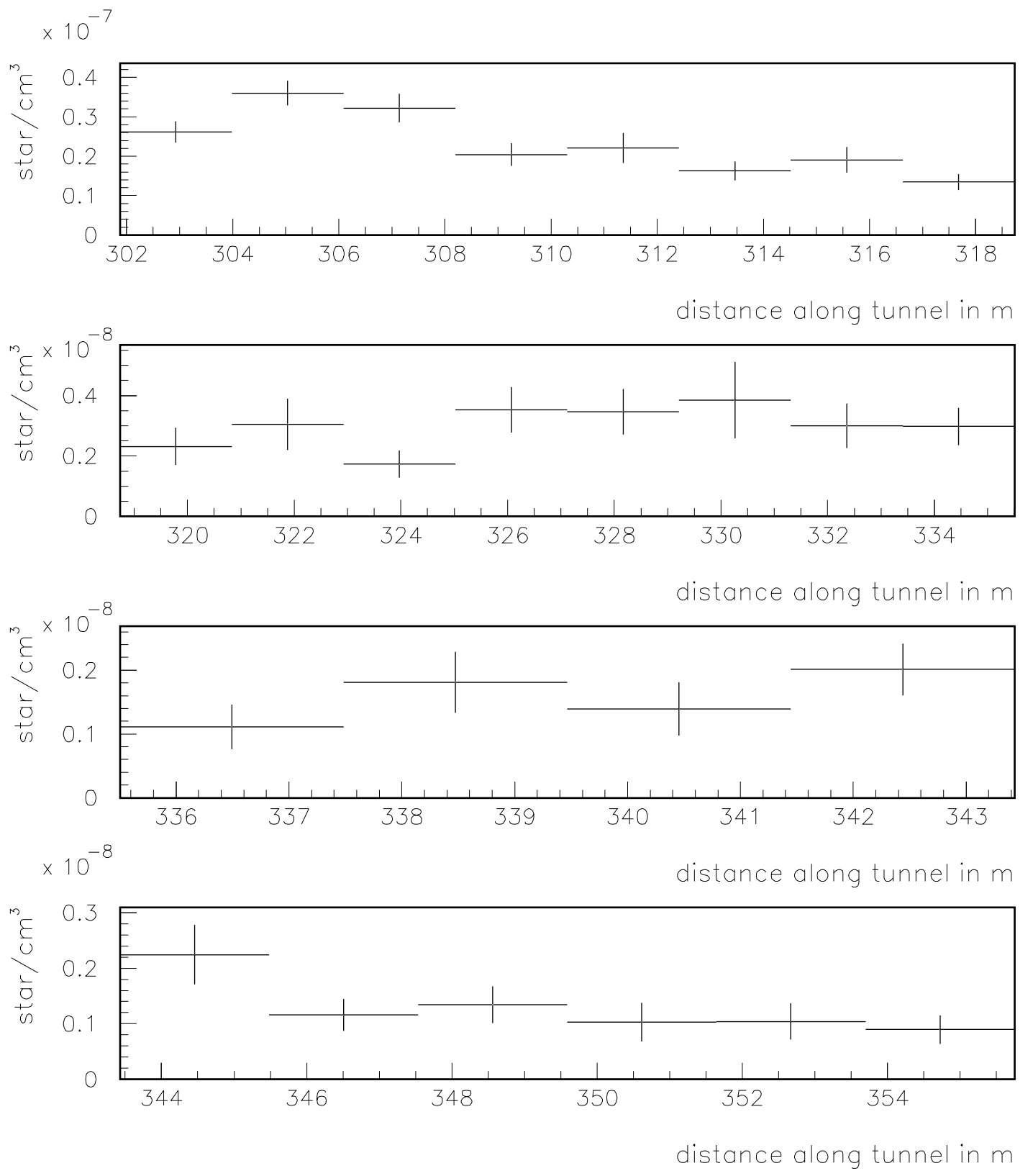

Star density distribution in $1 \mathrm{~cm}$ bin.

Figure B.30: Star density distribution for last four regions. Magnet current variation on $1.4 \%$ HV101 and $0.1 \%$ in V108. 

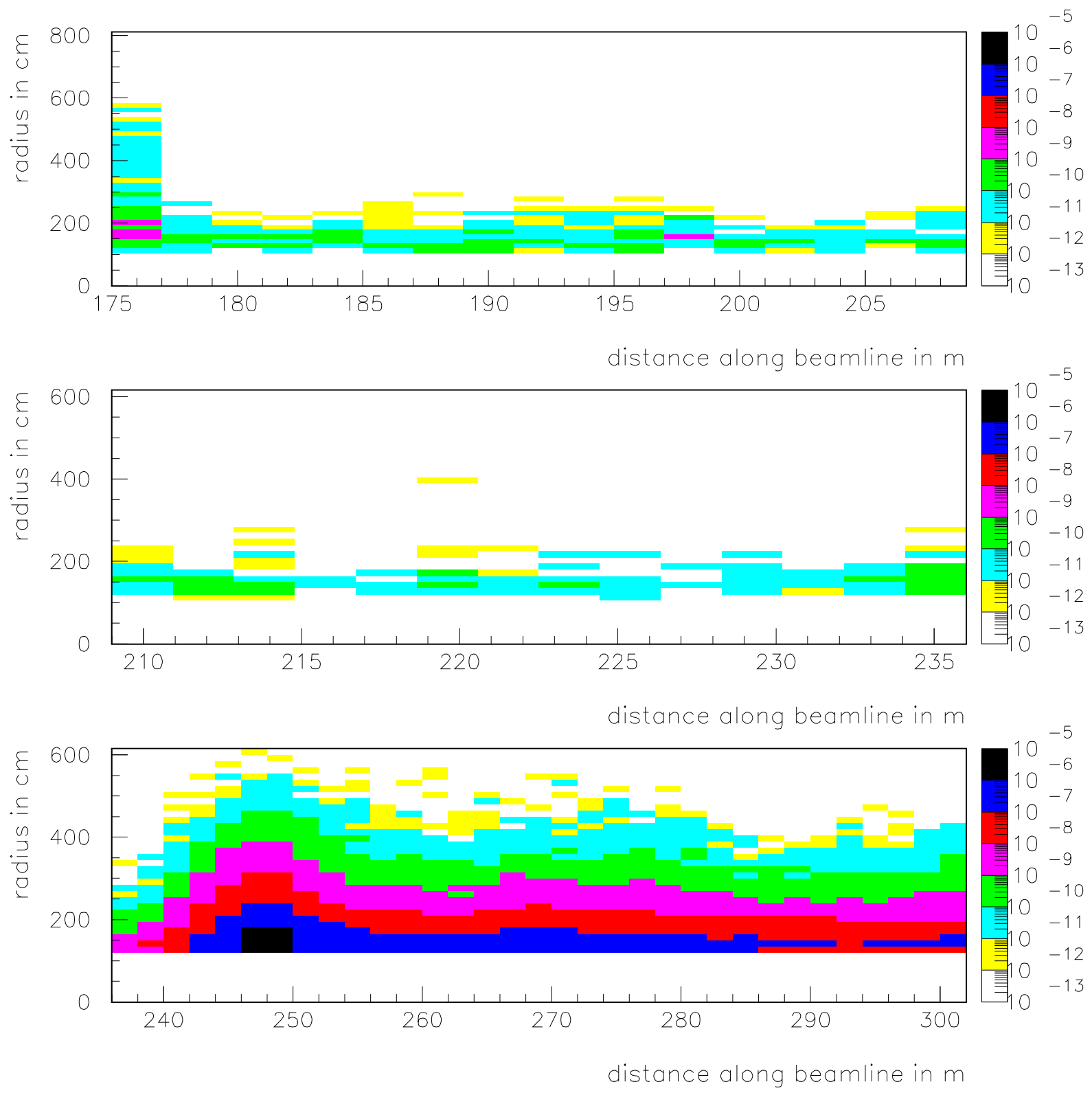

Star density. Magnetic field variation $0.8 \%$ in $\mathrm{V} 108$ and $0.5 \%$ in HV 101

Figure B.31: Star density distribution for first three regions. 

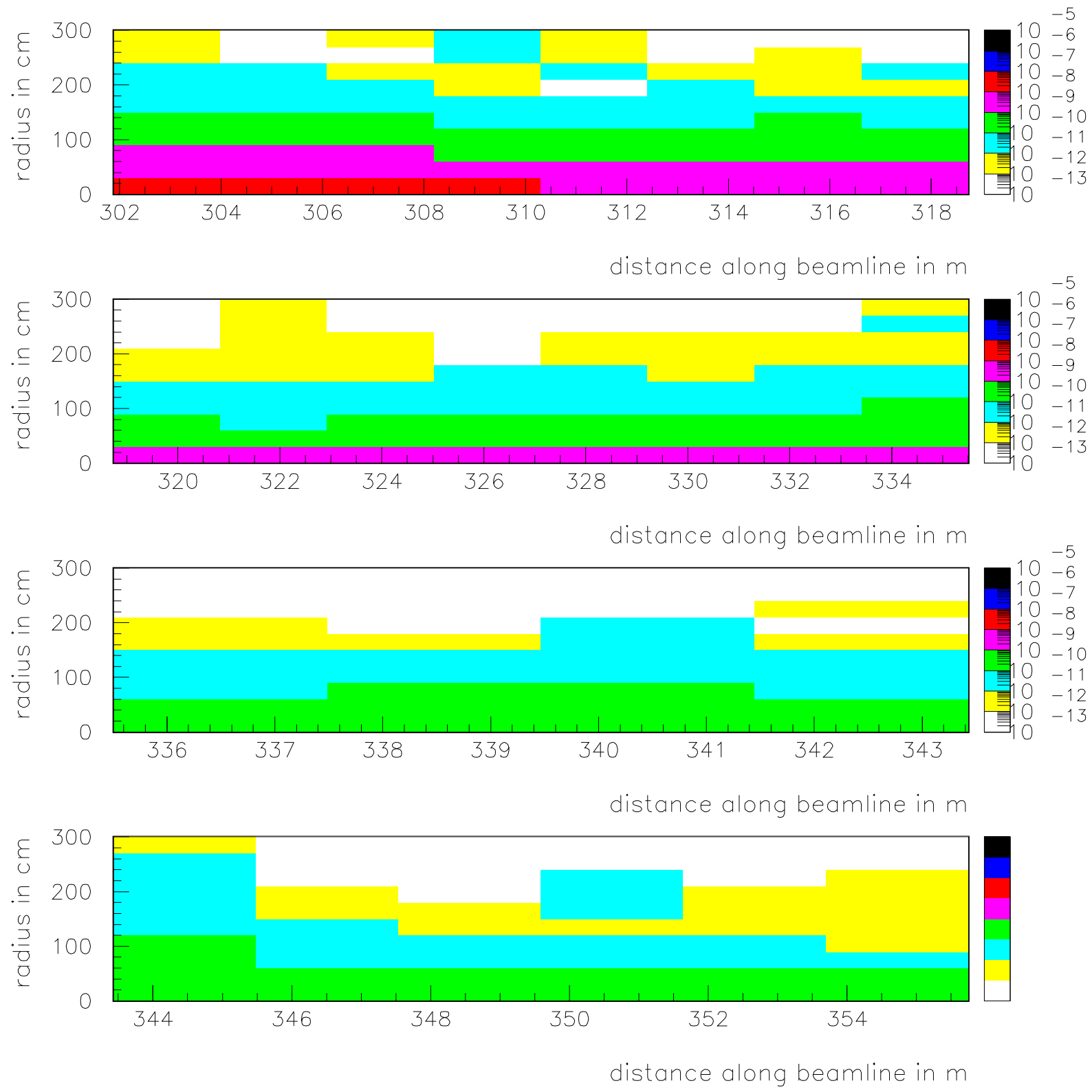

Star density. Magnetic field variation $0.8 \%$ in $\mathrm{V} 108$ and $0.5 \%$ in HV 101

Figure B.32: Star density distribution for last four regions. 


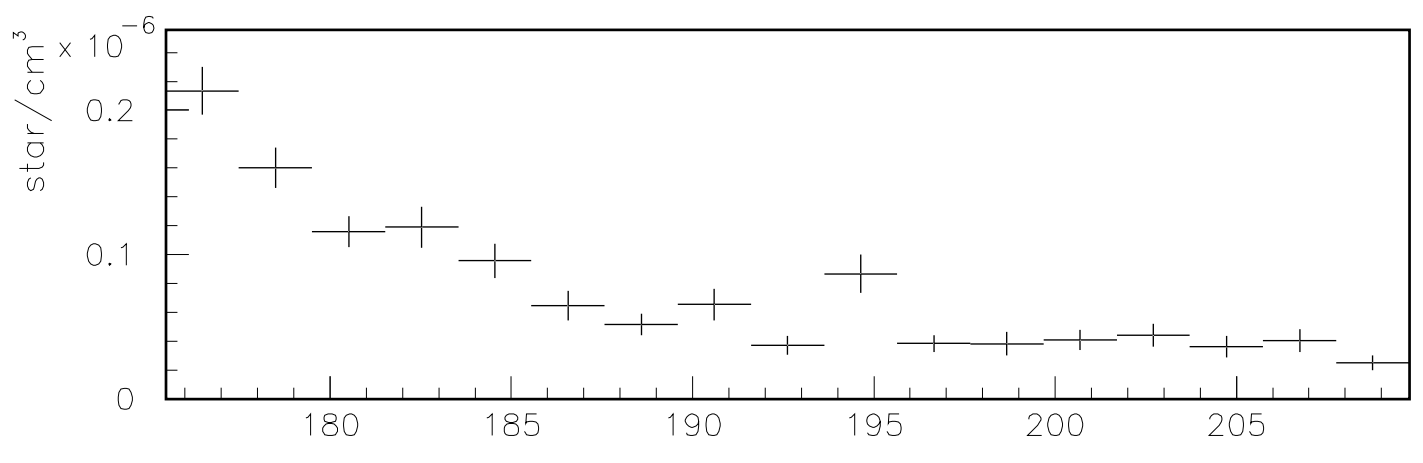

distance along tunnel in $m$
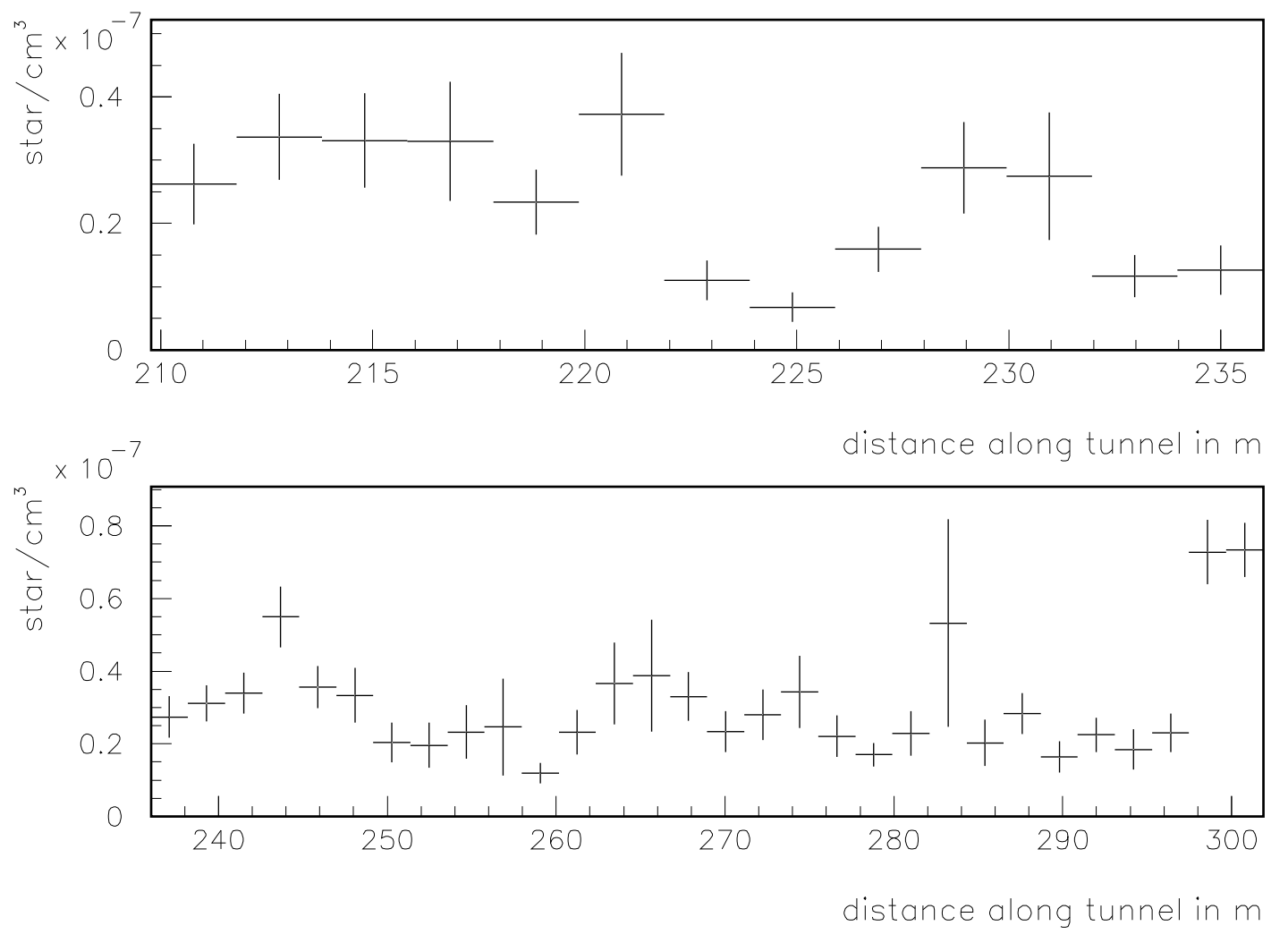

Star density distribution in $1 \mathrm{~cm}$ bin.

Figure B.33: Star density distribution for first three regions. Magnet current variation on $0.5 \%$ HV101 and $0.8 \%$ in V108. 

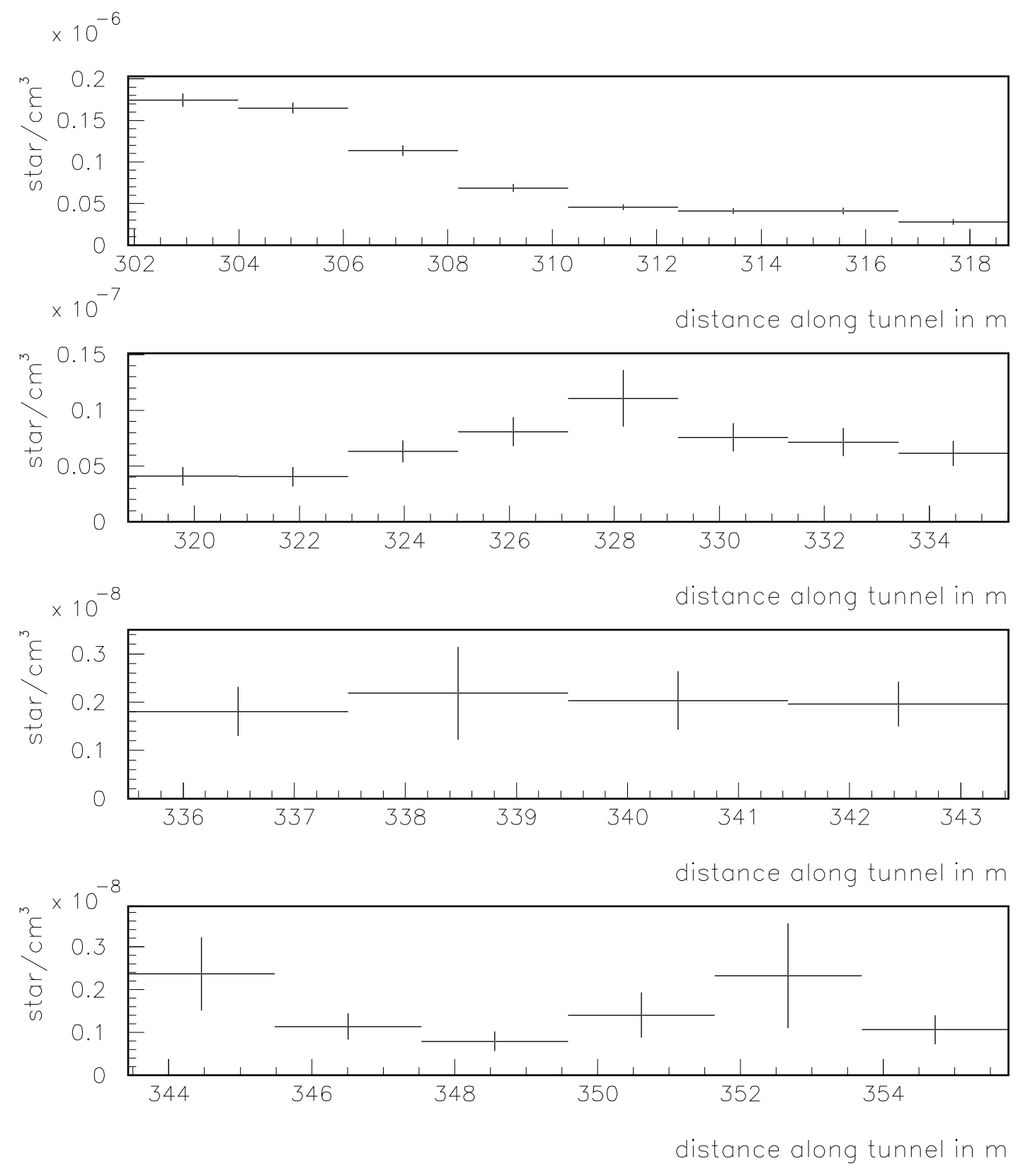

Star density distribution in $1 \mathrm{~cm}$ bin.

Figure B.34: Star density distribution for last four regions. Magnet current variation on $0.5 \%$ HV101 and $0.8 \%$ in V108. 

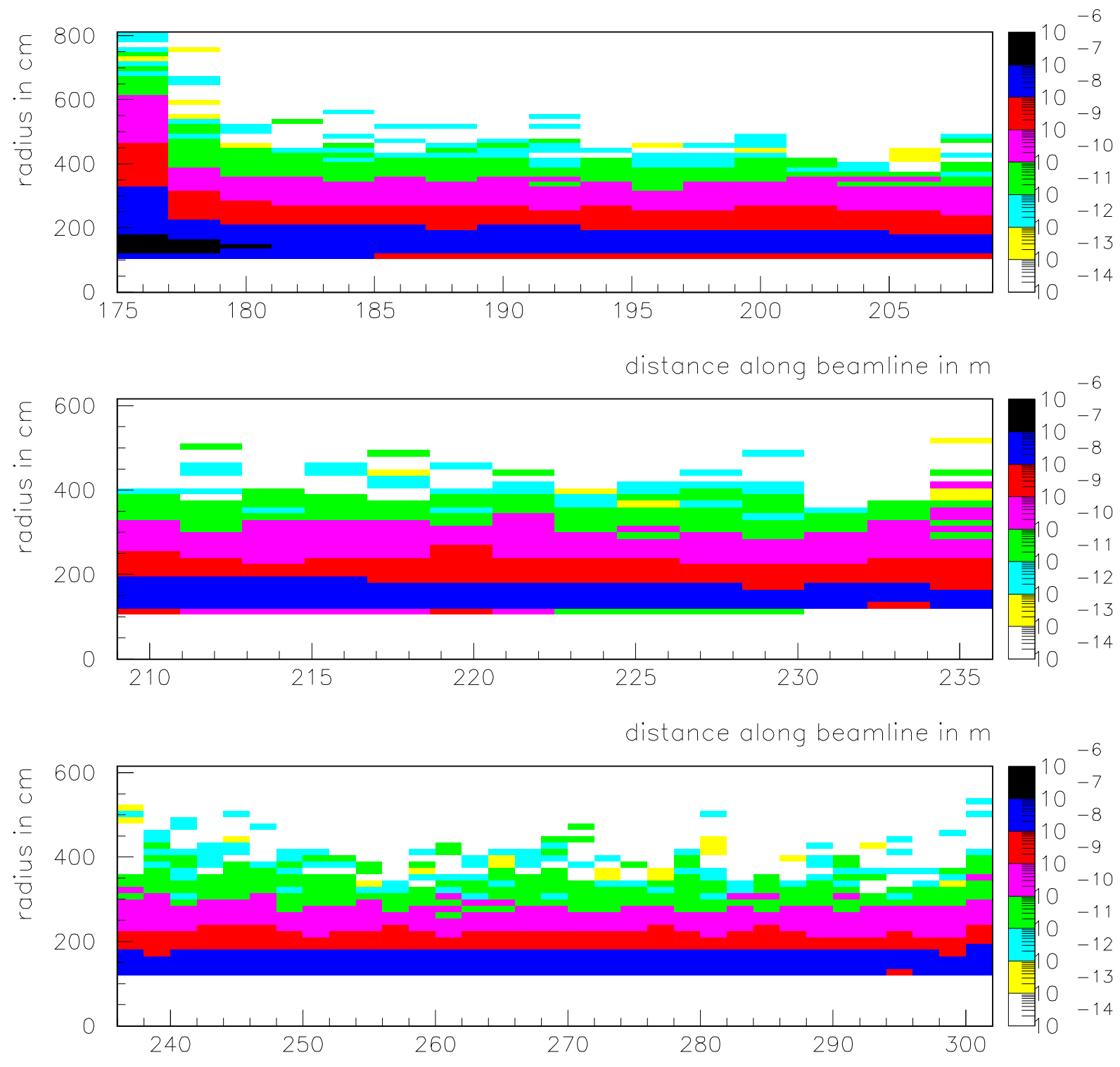

distance along beamline in $\mathrm{m}$

Star density. Magnetic field variation $0.1 \%$ in V108 and $1.4 \%$ in HV101

Figure B.35: Star density distribution for first three regions. 

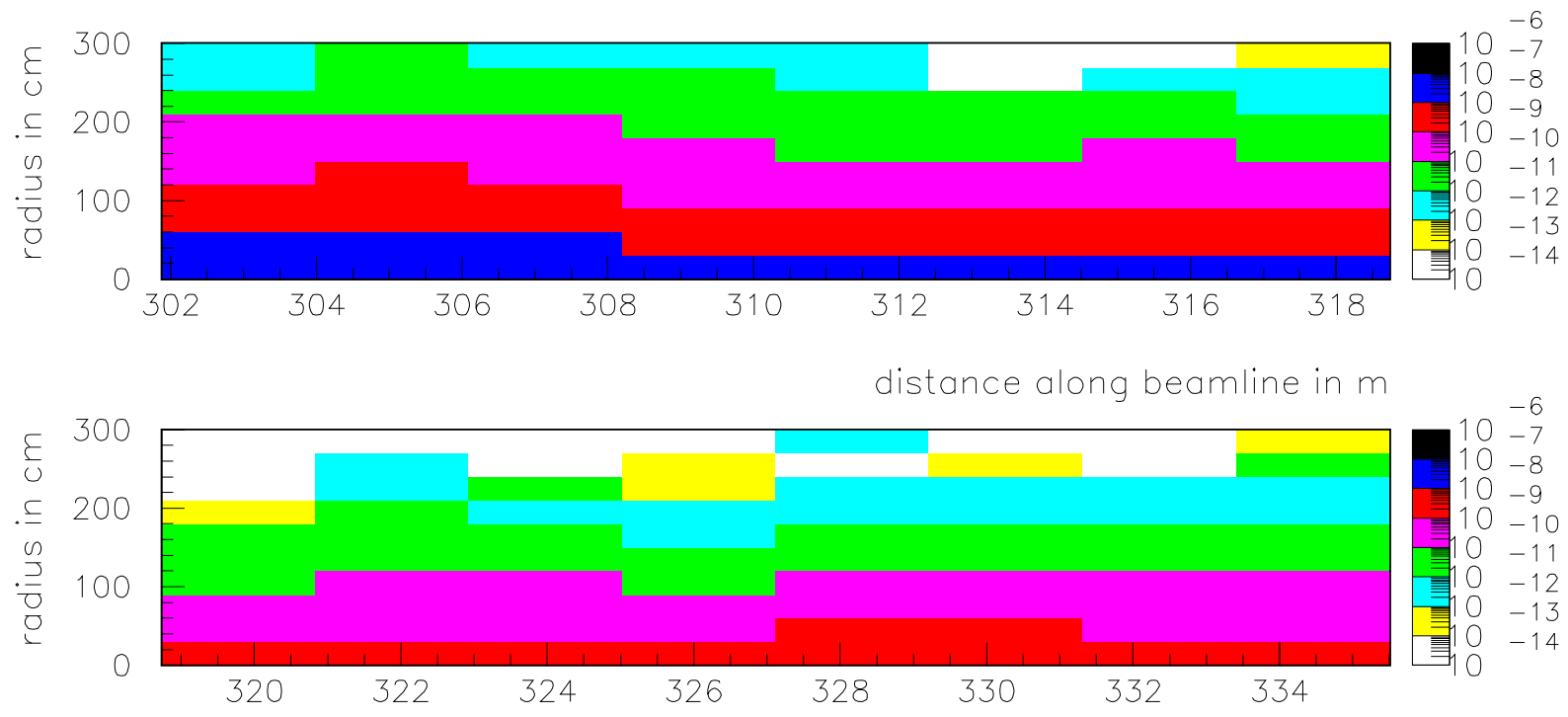

distance along beamline in $m$

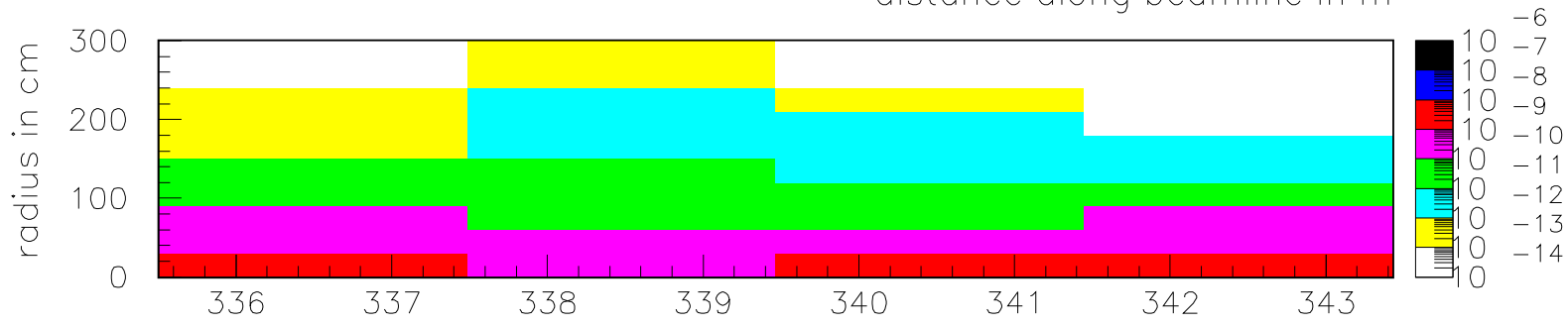

distance along beamline in $m$

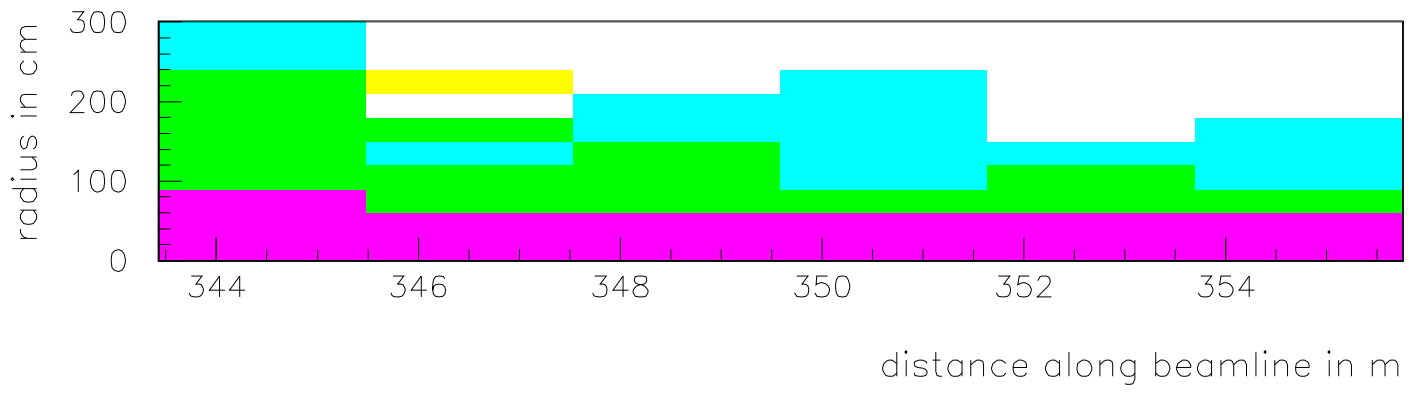

Star density. Magnetic field variation $0.1 \%$ in V108 and $1.4 \%$ in HV101

Figure B.36: Star density distribution for last four regions. 

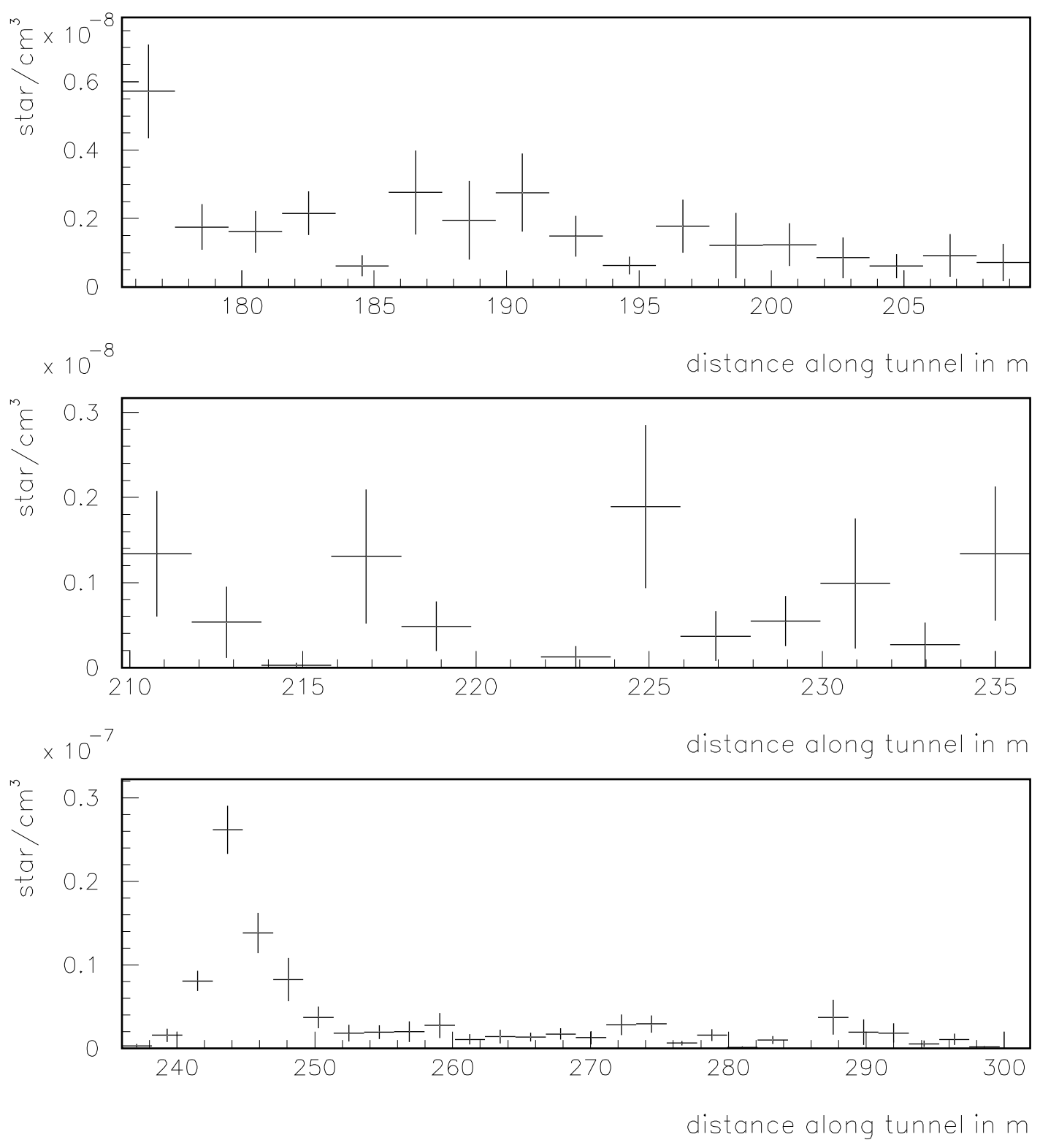

Star density distribution in $1 \mathrm{~cm}$ bin.

Figure B.37: Star density distribution for first three regions. Magnet current variation on $1 \% \mathrm{~V} 118$ and $0.7 \%$ in V108. 

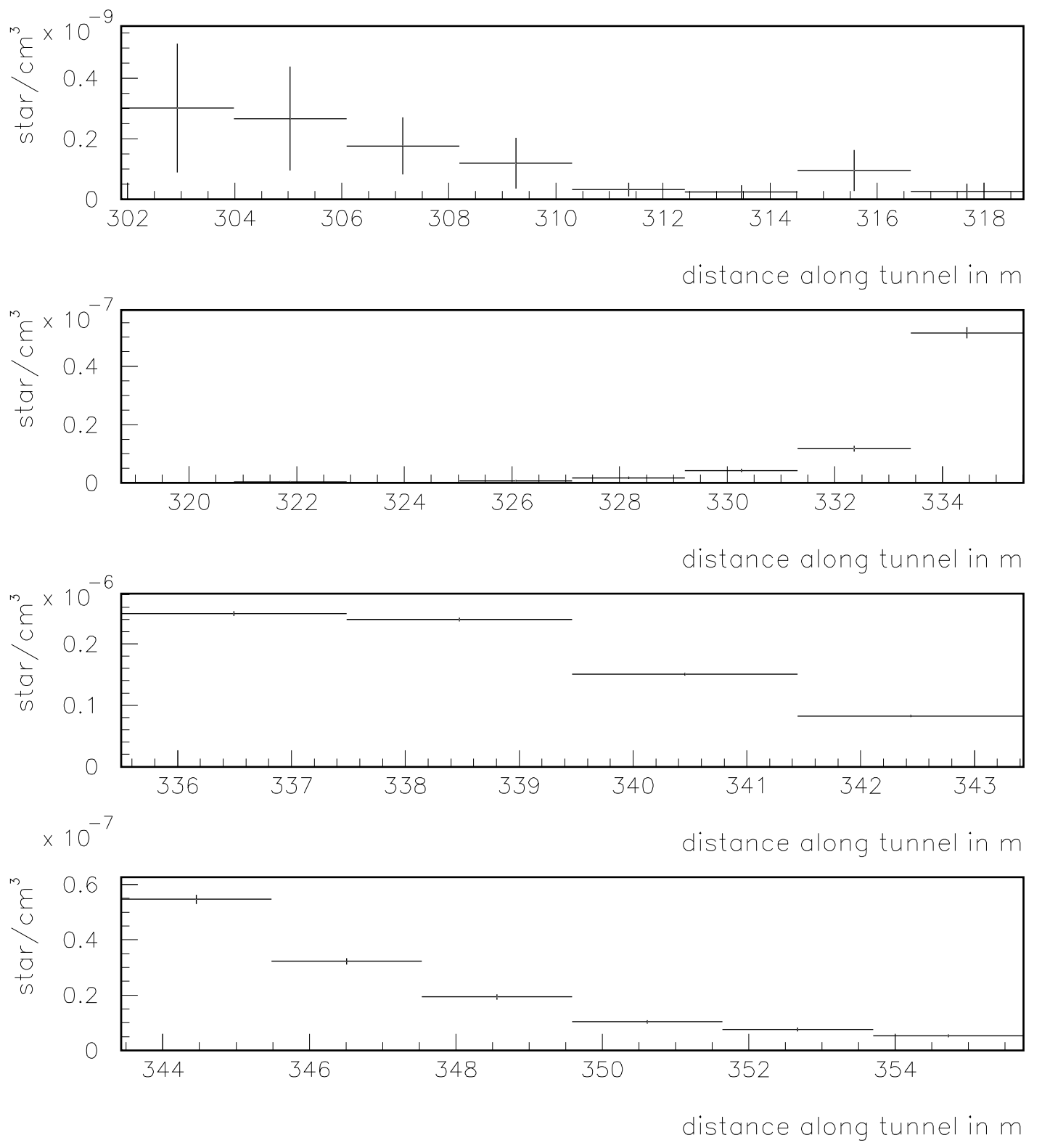

Star density distribution in $1 \mathrm{~cm}$ bin.

Figure B.38: Star density distribution for last four regions. Magnet current variation on $1 \% \mathrm{~V} 118$ and $0.7 \%$ in V108. 

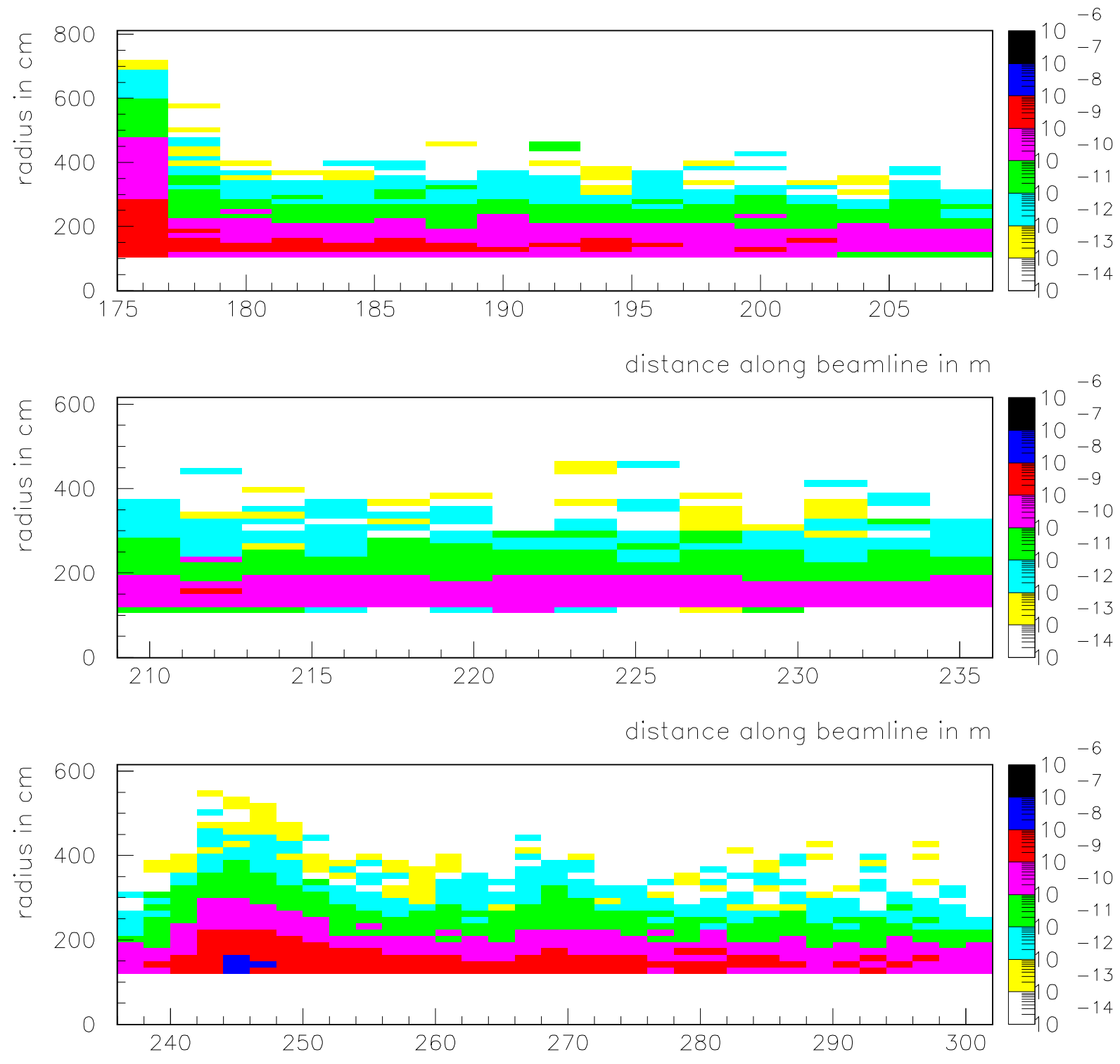

distance along beamline in $m$

Star density. Magnetic field variation $0.7 \%$ in V108 and $1 . \%$ in V118

Figure B.39: Star density distribution for first three regions. 

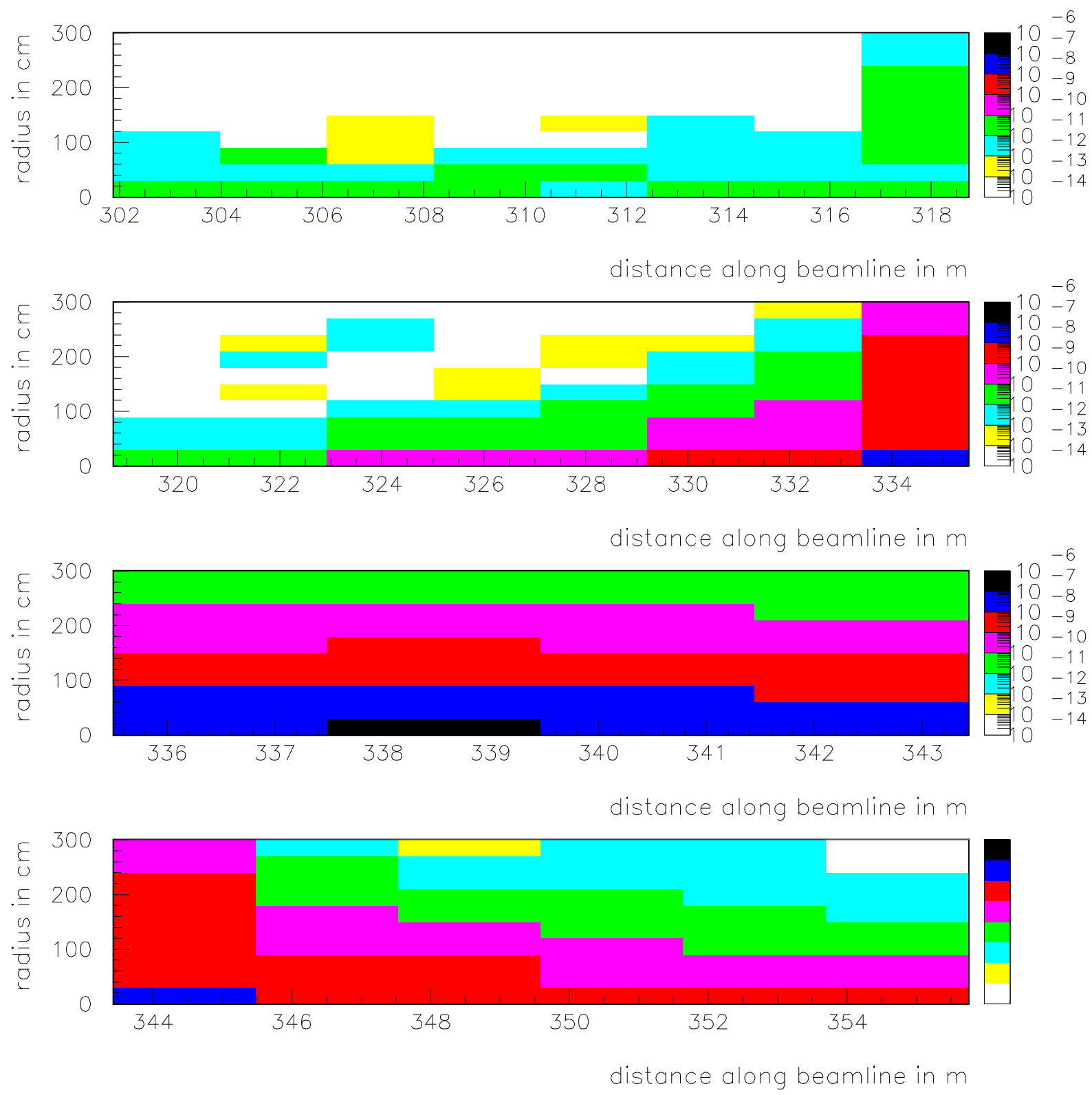

Star density. Magnetic field variation $0.7 \%$ in V108 and $1 \%$ in V1 18

Figure B.40: Star density distribution for last four regions. 


\section{Appendix C}

\section{Star Density and Beam Loss Monitors}

This appendix documents the studies of beam loss (studied in Chapter 4) to determine the simulated response in beam loss monitors at the position of each magnet. For most of the scenarios considered in Section 4.4, the energy deposition in beam loss monitors is calculated. Typical distributions of detector signals along the beam line are shown in Figure C.1. The energy deposition in the monitors and star density distribution are clearly correlated.

Monitor activation in first two regions is due to beam interactions with the HT107 corrector. In figure C.2 the dependence of average star density on the signal in beam loss monitor placed on HT107 is shown. The correlation can be described by a simple linear dependence.

In region 4 the most significant beam losses occur on the V110-1, Q108 and HT108 magnets. In figure C.3a the average star density vs. sum of signal from detectors placed on these magnets is shown. The V110-1 and Q108 magnets are much larger than the HT108 corrector (see Appendix A). Detectors set at different distances from beam pipe have different sensitivity. If the difference in sensitivity is taken into account, a linear correlation between star density and signal in detectors can be achieved (see figure C.3b) 


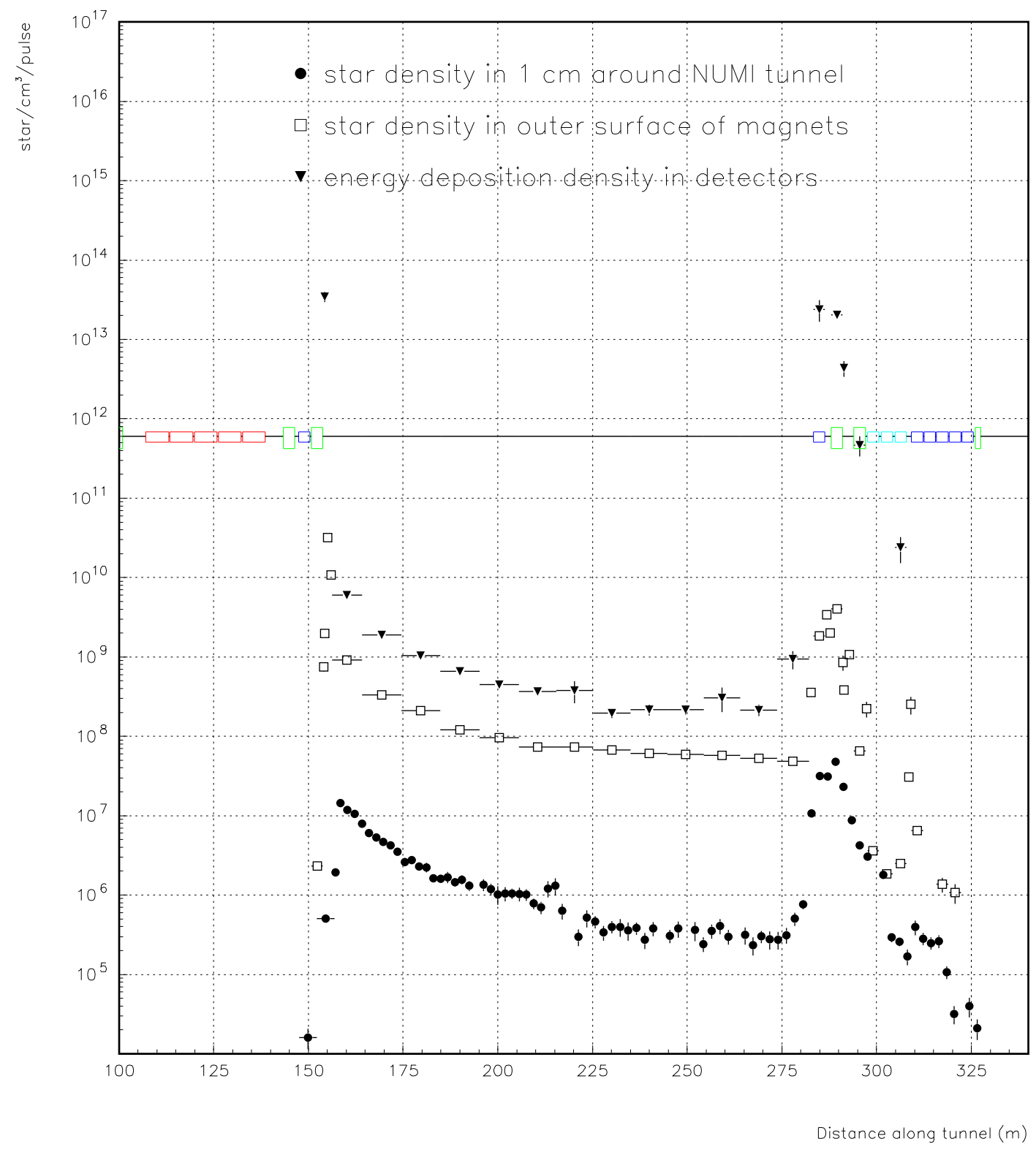

Figure C.1: Star density distribution and energy deposition in beam loss monitors. Magnet current variation set to $0.4 \%$ in V105. 


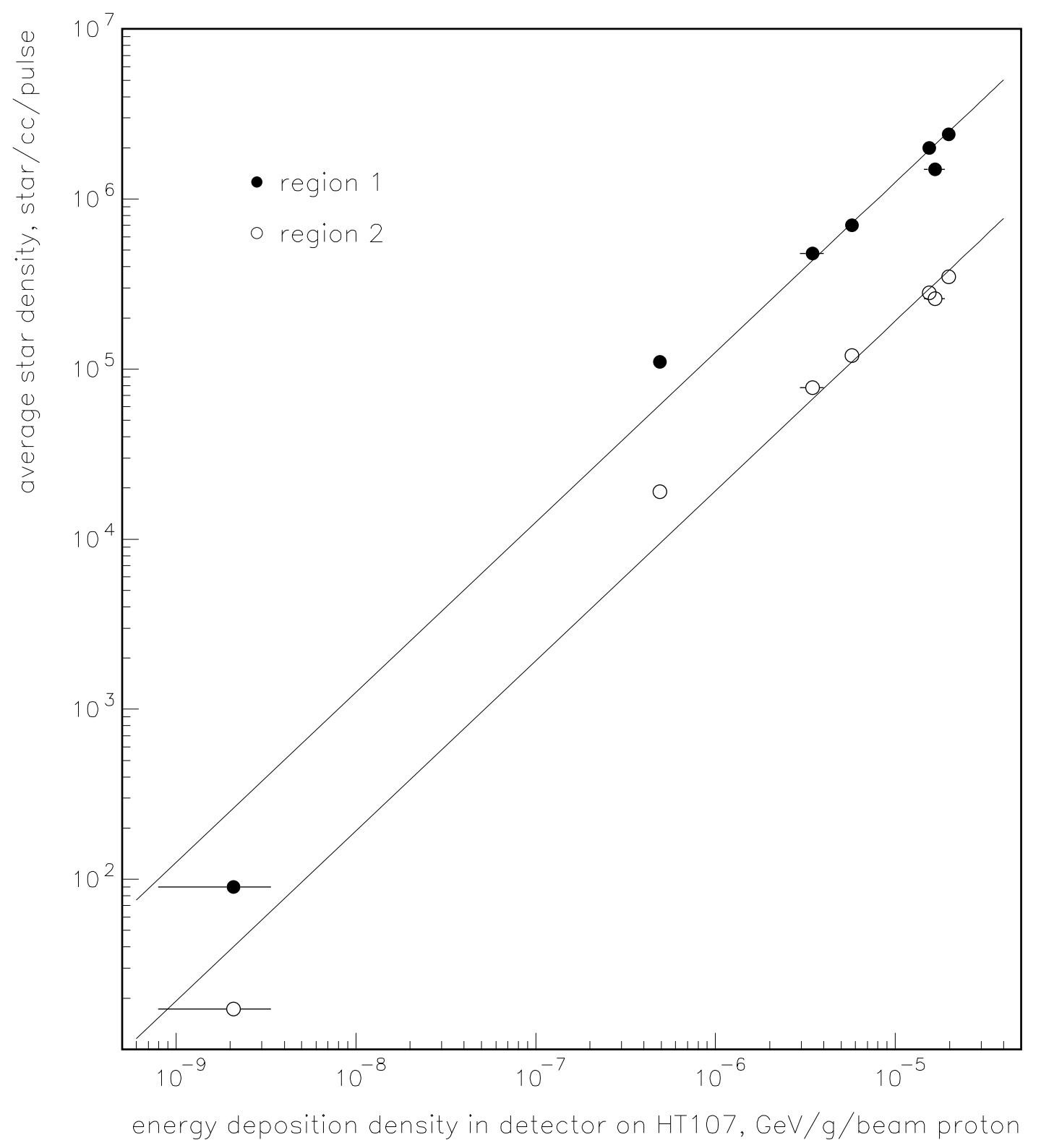

Figure C.2: Correlation between star density in regions 1,2 and energy deposition in beam loss monitor. 

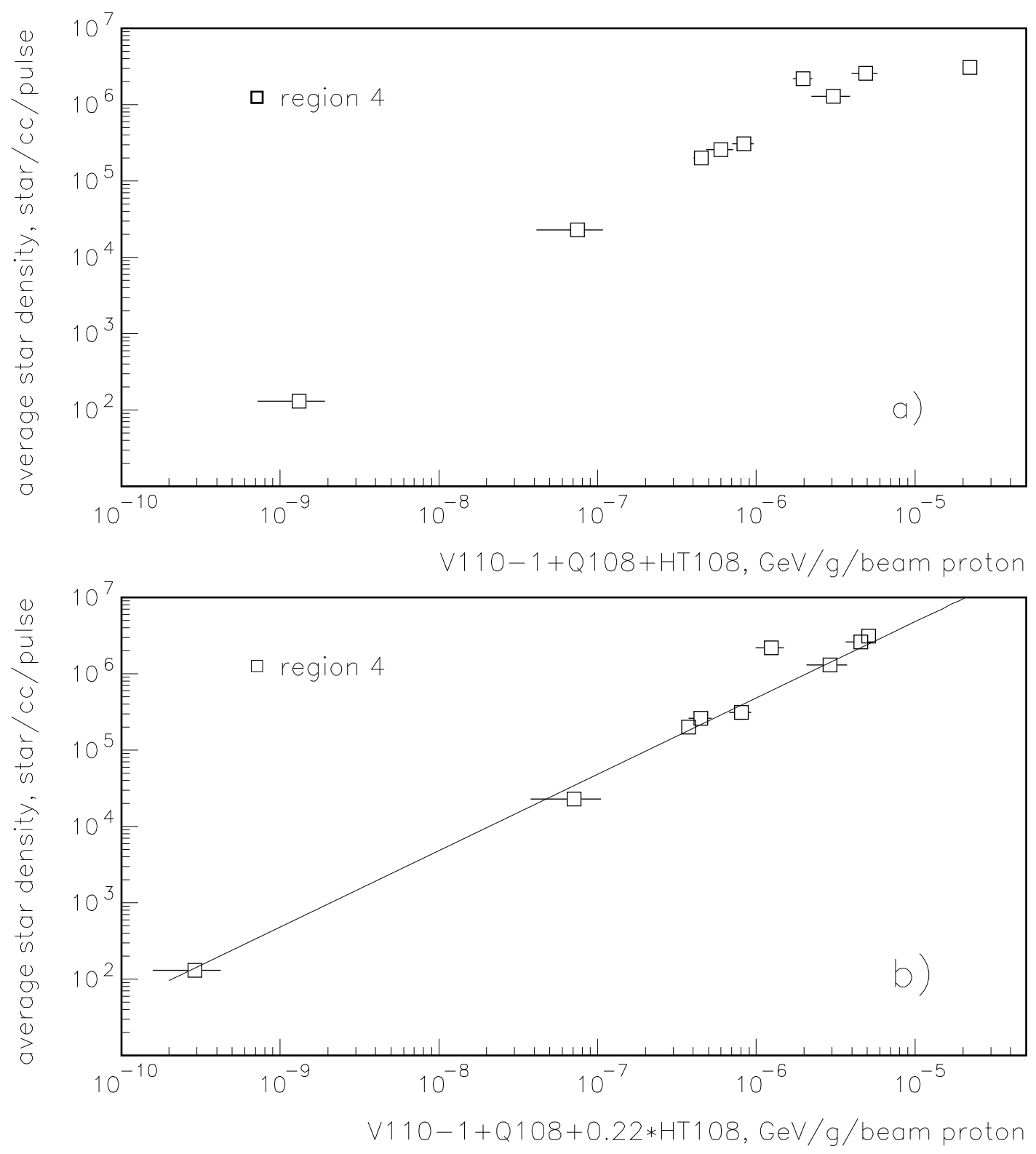

Figure C.3: Correlation between star density in region 4 and energy deposition in beam loss monitors. 